\title{
Modeling regional aerosol and aerosol precursor variability over California and its sensitivity to emissions and long-range transport during the 2010 CalNex and CARES campaigns
}

\author{
J. D. Fast et al.
}

Correspondence to: J. D. Fast (jerome.fast@pnnl.gov) 
This supplement contains additional tables and figures that summarize model performance at surface sampling sites and along aircraft flight paths and ship tracks. Statistical metrics in the tables include the bias, root-mean-square error (RMSE), correlation coefficient (R), and index of agreement (IA) for various meteorological, trace gas, and aerosol quantities.

\section{Aerosol Modeling Testbed}

The extensive data collected during CalNex and CARES are an ideal testbed for evaluating photochemical and aerosol models; therefore, they have been merged into a single dataset used by the Aerosol Modeling Testbed (AMT). The AMT [Fast et al., 2011] consists of a host model, testbed cases, and post-processing software. The host model is the Weather Research and Forecasting (WRF-Chem) community model [Skamarock et al., 2005] that permits on-line coupling of meteorology and chemistry [Grell et al., 2005; Fast et al., 2006]. Since detailed measurements of aerosol properties are not routinely collected aloft, the AMT uses meteorological, trace gas, and aerosol measurements from field campaigns to define each testbed case. The analysis software extracts simulated variables in a manner compatible with the available measurements using "instrument simulators". Examples of instrument simulators include the interpolation of model output in space and in time along research aircraft flight tracks and over vertical profiles sampled by radar wind profilers and lidars. Statistical and graphical programs are also available in the analysis software. While the AMT has been designed for use with WRF, the analysis software can be modified for other models. For example, Ensberg et al. [2013] used the AMT software coupled with the Community Multiscale Air Quality (CMAQ) modeling system to evaluate simulated aerosol concentration and composition in the Los Angeles Basin using the CalNex CIRPAS Twin Otter measurements.

The field campaign and operational data used for the CalNex/CARES testbed case have been provided through several archives with a variety of formats (e.g. ASCII, ICARTT, NetCDF, HDF, Microsoft Excel). Modelers need to write software that handles the variety of formats, which usually changes from field campaign to field campaign. Inevitably, each user that processes the same field dataset creates different software scripts or programs. As part of the AMT, a common ASCII format is employed for most of the data and data has been organized into common types including surface, aircraft, profile, and satellite. Subdirectories are created for each supersite or operational network in the case of surface instrumentation, and for each research aircraft in the case of airborne sampling. The directory structure for the AMT CalNex/CARES 
testbed case is given in Fig. S1. Some of the sub-directories contain data exclusively from CalNex or CARES, while other sub-directories contain data from both campaigns, e.g., in the case of instrument platforms participating in both, such as the B-200 and NOAA Twin Otter aircraft. In addition to data from the radiosonde, radar wind profiler, CARB, IMPROVE, AERONET networks, satellite measurements of aerosol optical depth from the Moderate Resolution Imaging Spectroradiometer (MODIS) on the Aqua and Terra satellites are also included in the testbed case. An identical directory structure is employed for model output extracted to be temporally and spatially compatible with the measurements, enabling graphics and statistics to evaluate model performance. In this way, the AMT permits users to spend more time on science issues (rather than on tedious and repetitive tasks associated with data processing), target specific aerosol processes and other atmospheric processes affecting aerosol evolution, and document improvements in parameterizations.

The testbed case containing the CARES and operational data as well as the analysis toolkit software is available to download from the Atmospheric Radiation Measurement (ARM) research climate facility archive at $h t t p: / / w w w . a r m . g o v / d a t a / e v a l / 59$. Users are encouraged to contact Jerome Fast (Jerome.Fast@pnnl.gov) for an updated version of the dataset.

\section{Evaluation of Meteorological Quantities}

Since the current model configuration is somewhat different than previous studies [Angevine et al., 2012; Fast et al., 2012], some comparisons of observed and simulated temperature, humidity, wind speed and direction, and solar radiation from the DEF_ANT simulation are presented here to demonstrate the performance of the model in representing the meteorological conditions that affect the vertical mixing, transport, chemical transformation, and removal of trace gases and aerosols.

An example of the simulated surface meteorology at the Pasadena supersite over May and June of 2010 as well as the diurnal averages is shown in Fig. S2. The model is able to reproduce the multi-day variability of temperature and relative humidity although the simulated nighttime temperatures are a few degrees warmer than observed and the relative humidity is generally $10 \%$ lower than observed at all times of the day. As seen in the solar radiation, the mostly sunny conditions occurred on the majority of the days. While the model correctly produces clouds on some days when they occurred (May 17 - 18, June 8-9, the overall reduction in downward shortwave radiation due to clouds is less than observed suggesting that the simulated liquid or ice water path is too low. The near-surface winds at Pasadena exhibit nearly the same diurnal variation from day to day, with southwesterly winds during the day that become 
weaker and southerly to southeasterly at night. While the model reproduces the diurnal variability in wind direction, the predicted wind speeds are too high. The over-prediction in wind speeds is likely due to two factors: 1) urban canopy effects that are not included in the current model configuration of the model, and 2) sub-grid scale terrain effects since the site is located near the edge of the San Gabriel Mountains. The performance of near surface winds is usually better at other stations located outside of urban areas and/or in flat terrain (not shown).

Table 4 summaries statistics that quantify model performance for the simulated nearsurface temperature, relative humidity, wind speed, and wind direction average over California and averaged over smaller geographic regions depicted in Fig. 1c. As with the Pasadena site, simulated temperatures are usually 0.2 to $0.9 \mathrm{~K}$ too low and the diurnal and multi-day temporal variations are similar to observations. Relatively humidity is usually lower than observed by 5.5 to $7.0 \%$ over the San Joaquin Valley and southern California, but is generally within $1 \%$ of the observations on average elsewhere. Simulated near surface wind speeds are usually higher than observed with the largest biases for stations in the "coastal" region. The average bias in wind direction is less than 10 degrees for the San Joaquin Valley, Sacramento Valley, and coastal regions, but between 17.6 and 22.5 degrees over southern California and the interior mountains. While the model qualitatively captures the diurnal and multi-day variability in wind direction, it is not surprising that it cannot represent the high frequency variations especially when wind speed are low $\left(<1 \mathrm{~m} \mathrm{~s}^{-1}\right)$ as shown in Fig. S2e. This is the primary reason for the low wind direction correlations in Table 4.

Statistics summarizing the performance in simulated temperature, relative humidity, wind speed, and wind direction for all of the G-1, CIRPAS Twin Otter, and WP-3D flight paths and the $\mathrm{R} / \mathrm{V}$ Atlantis deployment are shown in Table 5. In general, the spatial and temporal variability in temperature in the lower troposphere is reasonably simulated as reflected by the relatively high correlation coefficients that are similar to the surface measurements; however, the model is 2 to 3 degrees colder than observed on average. Conversely, the near surface temperatures over the ocean are about 1 degree warmer on average than those from the R/V Atlantis. The relative humidity statistics are similar to those at the surface measurement sites with the model being 4 to $7 \%$ drier than observed, except along the G-1 flight paths that had a very small bias. The wind speeds aloft along the G-1 and WP-3D flight paths are very similar to observed and consequently the errors are much smaller than at the surface measurement sites. Nevertheless, the correlation coefficients indicate that the model did not represent all of the spatial and temporal variability in wind speed. Wind speed variability is better represented over southern California where the WP- 
3D usually flew than over northern California where the G-1 flew. Statistics for individual aircraft flights and daily statistics for the R/V Atlantis sampling are given in Tables S1 - S14.

It is also important to evaluate the evolving simulated winds throughout the boundary layer and lower troposphere when assessing the ability of a model to simulate horizontal transport downwind of emissions sources; therefore the simulated winds aloft have been evaluated with measurements from the radar wind profiler network shown in Fig. 1c. While Fast et al. [2012] demonstrated that the observed and simulated wind speed and direction, associated with varying synoptic conditions and thermally-driven flows, was similar in the vicinity of Sacramento during June, this study quantifies model performance over all of California for May and June. Statistics that summarize the model performance at all the radar wind profiler sites at three altitudes are given in Tables 6 and 7. The performance varies among the sites and with altitude as expected. Figure S3 shows the observed and simulated diurnally-averaged winds over May and June of 2010 at the Sacramento, Bakersfield, and USC radar wind profilers based on the time series shown in Fig. S4. The simulated wind speed and direction at Sacramento (Fig. S2a) is very close to observed, except for a few periods during the night that differ by as much as 30 degrees. Both the observed and simulated winds at Bakersfield are usually northwesterly all day (Fig S2b), but the model overestimates the wind speeds at night. Low-level jets frequently occur in the San Joaquin Valley, but the simulated wind speeds are too strong on some nights consistent with Bao et al. [2008] in their WRF simulation of winds during the Central California Ozone Study. At USC (Fig. S2c), the model reproduces the overall diurnal variation in wind speed and direction at this altitude, but the wind speeds are generally 1-2 $\mathrm{m} \mathrm{s}^{-1}$ higher than observed during the night and early morning and the simulated wind directions are more westerly than observed. When comparing Fig. S2d and Fig. S4c, the daytime wind speed bias decreases significantly with height, but the bias at night is similar at both altitudes.

Boundary-layer depth is an important meteorological quantity, since it defines the vertical extent of turbulent mixing that dilutes the concentrations of near-surface trace gases and aerosols and alters chemical transformation. The performance of the model in simulated boundary-layer depth compared with the radiosondes collected at the T0 and T1 sites is nearly identical to Fast et al. [2012] and is not included here. Scarino et al. [2013] present a methodology of deriving boundary layer heights from backscatter profiles measured by the HRSL on the B-200 aircraft. An advantage of this data set is that the simulated spatial and temporal variability in boundary layer height can be evaluated, as opposed to comparing model predictions to infrequent soundings made at a few locations. Scarino et al. [2013] use the results from the DEF_ANT simulation to show that the simulated spatial and temporal variations in boundary layer depths are usually 
similar to those derived along the B-200 aircraft flight paths. Statistics that summarize the model performance during the day also show that the model boundary layer depths are somewhat too low over southern California during the CalNex flights, but are closer to observed over northern California during the CARES flights. 
Table S1. Performance of simulated temperature (T) in terms of bias, RMSE, R, and IA for the individual G-1 aircraft flight paths.

\begin{tabular}{cccccccc}
\hline \multirow{2}{*}{ Variable } & $\begin{array}{c}\text { Flight } \\
\text { Date }\end{array}$ & $\begin{array}{c}\text { Number of } \\
\text { Data Points }\end{array}$ & $\begin{array}{c}\text { Observed } \\
\text { Mean }\end{array}$ & Bias & RMSE & R & IA \\
\hline \multirow{5}{*}{ June 03a } & 849 & 292.0 & -2.9 & 3.1 & 0.96 & 0.69 \\
& June 06a & 1164 & 296.3 & -4.0 & 4.3 & 0.39 & 0.33 \\
& June 06b & 989 & 296.1 & -4.5 & 4.7 & 0.97 & 0.85 \\
& June 08a & 1170 & 293.2 & -2.4 & 3.3 & 0.27 & 0.47 \\
& June 08b & 1213 & 292.7 & -5.0 & 5.5 & 0.95 & 0.73 \\
& June 10a & 1113 & 290.0 & -1.7 & 2.2 & 0.93 & 0.91 \\
& June 12a & 1093 & 293.8 & -2.7 & 3.3 & 0.67 & 0.63 \\
& June 12b & 1001 & 298.0 & -1.7 & 2.2 & 0.90 & 0.85 \\
& June 14a & 1168 & 296.6 & -1.7 & 2.3 & 0.73 & 0.75 \\
& June 15a & 1103 & 290.2 & -1.1 & 2.2 & 0.05 & 0.42 \\
& June 15b & 1116 & 292.1 & -3.8 & 5.1 & 0.94 & 0.82 \\
& June 18a & 1164 & 293.2 & -0.2 & 2.5 & 0.93 & 0.96 \\
& June 19a & 1120 & 290.9 & -2.3 & 3.2 & 0.90 & 0.83 \\
& June 21a & 1130 & 291.8 & -2.0 & 2.8 & -0.17 & 0.38 \\
& June 21b & 1126 & 296.6 & -2.7 & 3.0 & 0.98 & 0.95 \\
& June 23a & 1180 & 292.6 & -2.4 & 3.8 & 0.90 & 0.85 \\
June 23b & 1106 & 296.1 & -3.2 & 4.5 & 0.93 & 0.87 \\
June 24a & 1173 & 292.3 & 1.4 & 3.6 & -0.12 & 0.34 \\
June 24b & 1034 & 294.7 & -3.7 & 5.0 & 0.92 & 0.76 \\
June 27a & 1215 & 298.7 & -1.7 & 2.2 & 0.80 & 0.79 \\
June 28a & 1139 & 301.3 & -2.5 & 2.9 & 0.85 & 0.74 \\
June 28b & 847 & 306.0 & -0.9 & 2.3 & 0.88 & 0.89 \\
\hline
\end{tabular}


Table S2. Performance of simulated relative humidity (RH) in terms of bias, RMSE, R, and IA for the individual G-1 aircraft flight paths.

\begin{tabular}{cccccccc}
\hline \multirow{2}{*}{ Variable } & $\begin{array}{c}\text { Flight } \\
\text { Date }\end{array}$ & $\begin{array}{c}\text { Number of } \\
\text { Data Points }\end{array}$ & $\begin{array}{c}\text { Observed } \\
\text { Mean }\end{array}$ & Bias & RMSE & R & IA \\
\hline & June 03a & 849 & 60.2 & 7.2 & 10.9 & 0.22 & 0.47 \\
& June 06a & 1117 & 55.5 & 6.9 & 9.1 & 0.23 & 0.42 \\
& June 06b & 988 & 41.3 & 10.4 & 18.8 & -0.20 & 0.23 \\
& June 08a & 1170 & 56.3 & 3.9 & 9.4 & 0.55 & 0.70 \\
& June 08b & 1212 & 40.1 & -1.8 & 19.4 & 0.20 & 0.49 \\
& June 10a & 1111 & 38.9 & 1.2 & 12.0 & 0.18 & 0.51 \\
& June 12a & 1093 & 28.2 & -1.8 & 5.3 & -0.16 & 0.26 \\
& June 12b & 991 & 25.5 & 2.3 & 4.7 & 0.56 & 0.67 \\
& June 14a & 1168 & 32.2 & 2.6 & 9.6 & 0.54 & 0.70 \\
& June 15a & 1103 & 55.4 & 2.5 & 13.3 & 0.71 & 0.79 \\
June 15b & 1115 & 42.4 & -4.0 & 20.7 & 0.24 & 0.44 \\
& June 18a & 1142 & 25.2 & -5.1 & 10.5 & 0.68 & 0.77 \\
& June 19a & 1097 & 39.3 & 2.7 & 11.8 & 0.35 & 0.60 \\
& June 21a & 1130 & 43.4 & -5.1 & 8.2 & 0.56 & 0.65 \\
& June 21b & 1126 & 21.4 & 2.0 & 6.6 & 0.63 & 0.78 \\
& June 23a & 1180 & 40.5 & -6.2 & 15.3 & 0.28 & 0.56 \\
June 23b & 1077 & 30.5 & 0.7 & 14.4 & -0.01 & 0.42 \\
June 24a & 1173 & 44.3 & -5.5 & 18.6 & -0.08 & 0.35 \\
June 24b & 1005 & 37.3 & 1.4 & 13.7 & 0.13 & 0.42 \\
June 27a & 1215 & 41.4 & -9.6 & 16.0 & 0.37 & 0.59 \\
June 28a & 1136 & 38.3 & 2.2 & 7.9 & 0.51 & 0.71 \\
June 28b & 843 & 25.2 & -0.4 & 4.9 & 0.58 & 0.73 \\
\hline
\end{tabular}


Table S3. Performance of simulated wind speed (WS) in terms of bias, RMSE, R, and IA for the individual G-1 aircraft flight paths.

\begin{tabular}{cccccccc}
\hline \multirow{2}{*}{ Variable } & $\begin{array}{c}\text { Flight } \\
\text { Date }\end{array}$ & $\begin{array}{c}\text { Number of } \\
\text { Data Points }\end{array}$ & $\begin{array}{c}\text { Observed } \\
\text { Mean }\end{array}$ & Bias & RMSE & R & IA \\
\hline June 03a & 849 & 7.0 & -1.3 & 3.3 & -0.16 & 0.35 \\
& June 06a & 1117 & 3.5 & -0.2 & 1.8 & -0.01 & 0.36 \\
& June 06b & 989 & 6.1 & -0.4 & 2.6 & 0.81 & 0.90 \\
& June 08a & 1170 & 3.8 & -1.2 & 2.3 & 0.23 & 0.52 \\
& June 08b & 1213 & 5.3 & -0.2 & 1.9 & 0.60 & 0.77 \\
June 10a & 1112 & 8.0 & 0.5 & 1.8 & 0.55 & 0.70 \\
June 12a & 1093 & 11.0 & 0.1 & 4.1 & 0.53 & 0.66 \\
June 12b & 1001 & 9.8 & -1.6 & 3.9 & -0.15 & 0.27 \\
June 14a $\left(m \mathrm{~m} \mathrm{~s}^{-1}\right)$ & 1168 & 4.5 & 0.1 & 3.2 & -0.23 & 0.27 \\
& June 15a & 1103 & 4.6 & 1.0 & 2.5 & 0.17 & 0.49 \\
June 15b & 1115 & 5.5 & 0.8 & 2.6 & 0.54 & 0.70 \\
June 18a & 1142 & 6.3 & -2.0 & 3.6 & 0.23 & 0.50 \\
June 19a & 1097 & 5.7 & -0.5 & 2.1 & -0.01 & 0.39 \\
June 21a & 1130 & 4.5 & 2.7 & 4.3 & 0.60 & 0.66 \\
June 21b & 1060 & 6.1 & -2.0 & 12.3 & 0.06 & 0.11 \\
June 23a & 1180 & 3.5 & 0.1 & 2.6 & 0.55 & 0.67 \\
June 23b & 1077 & 3.9 & 0.8 & 2.6 & 0.35 & 0.56 \\
June 24a & 1173 & 5.8 & -2.0 & 3.4 & 0.37 & 0.57 \\
June 24b & 1005 & 4.9 & 1.5 & 2.8 & -0.06 & 0.32 \\
June 27a & 1215 & 2.4 & -0.2 & 1.9 & -0.14 & 0.30 \\
June 28a & 1136 & 2.8 & -0.2 & 2.7 & 0.14 & 0.45 \\
June 28b & 843 & 4.3 & -1.2 & 2.4 & -0.01 & 0.42 \\
\hline
\end{tabular}


Table S4. Performance of simulated wind direction (WD) in terms of bias, RMSE, R, and IA for the individual G-1 aircraft flight paths.

\begin{tabular}{cccccccc}
\hline \multirow{2}{*}{ Variable } & $\begin{array}{c}\text { Flight } \\
\text { Date }\end{array}$ & $\begin{array}{c}\text { Number of } \\
\text { Data Points }\end{array}$ & $\begin{array}{c}\text { Observed } \\
\text { Mean }\end{array}$ & Bias & RMSE & R & IA \\
\hline June 03a & 849 & 225.0 & 31.1 & 41.3 & 0.41 & 0.50 \\
& June 06a & 1117 & 255.0 & 1.0 & 48.3 & 0.21 & 0.55 \\
& June 06b & 989 & 255.0 & 27.4 & 47.8 & 0.28 & 0.51 \\
& June 08a & 1170 & 165.0 & 45.8 & 83.7 & 0.31 & 0.64 \\
& June 08b & 1213 & 195.0 & 4.5 & 39.2 & 0.01 & 0.39 \\
June 10a & 1112 & 315.0 & -0.2 & 18.3 & -0.03 & 0.55 \\
June 12a & 1093 & 345.0 & 21.9 & 34.8 & 0.42 & 0.99 \\
June 12b & 1001 & 315.0 & 15.6 & 26.7 & 0.08 & 0.75 \\
& June 14a & 1168 & 195.0 & 15.2 & 63.2 & 0.12 & 0.50 \\
June 15a & 1103 & 195.0 & 4.9 & 40.4 & 0.61 & 0.79 \\
June 15b & 1115 & 195.0 & 8.3 & 31.2 & 0.25 & 0.54 \\
June 18a & 1142 & 315.0 & 16.2 & 51.8 & 0.71 & 0.78 \\
June 19a & 1097 & 195.0 & 3.7 & 20.2 & 0.55 & 0.73 \\
June 21a & 1130 & 345.0 & 2.2 & 62.8 & 0.57 & 0.83 \\
June 21b & 1060 & 285.0 & -6.4 & 40.5 & 0.07 & 0.89 \\
June 23a & 1180 & 165.0 & 2.5 & 72.7 & -0.11 & 0.69 \\
June 23b & 1077 & 255.0 & -0.4 & 53.8 & 0.17 & 0.49 \\
June 24a & 1173 & 165.0 & 27.6 & 49.9 & 0.58 & 0.72 \\
June 24b & 1005 & 165.0 & -5.7 & 39.1 & 0.30 & 0.51 \\
June 27a & 1215 & 285.0 & 12.2 & 100.3 & -0.09 & 0.75 \\
June 28a & 1136 & 255.0 & -49.2 & 108.2 & -0.12 & 0.59 \\
June 28b & 843 & 255.0 & 45.1 & 70.5 & -0.08 & 0.47 \\
\hline
\end{tabular}


Table S5. Performance of simulated temperature (T) in terms of bias, RMSE, R, and IA for the individual WP-3D aircraft flight paths.

\begin{tabular}{cccccccc}
\hline \multirow{2}{*}{ Variable } & $\begin{array}{c}\text { Flight } \\
\text { Date }\end{array}$ & $\begin{array}{c}\text { Number of } \\
\text { Data Points }\end{array}$ & $\begin{array}{c}\text { Observed } \\
\text { Mean }\end{array}$ & Bias & RMSE & R & IA \\
\hline \multirow{5}{*}{ May 04 } & 17219 & 287.5 & -4.7 & 6.0 & 0.88 & 0.85 \\
& May 07 & 24839 & 288.3 & -5.5 & 6.4 & 0.90 & 0.84 \\
& May 08 & 25439 & 286.6 & -3.6 & 5.1 & 0.87 & 0.86 \\
& May 11 & 25799 & 275.8 & -5.2 & 6.1 & 0.97 & 0.95 \\
& May 12 & 27489 & 285.1 & -4.7 & 6.9 & 0.91 & 0.89 \\
& May 14 & 22259 & 285.6 & -0.4 & 3.9 & 0.87 & 0.93 \\
& May 16 & 27899 & 286.5 & -2.8 & 4.4 & 0.87 & 0.89 \\
& May 19 & 24239 & 287.0 & -3.2 & 4.8 & 0.86 & 0.87 \\
& May 21 & 10722 & 286.8 & 0.4 & 1.5 & 0.82 & 0.89 \\
& May 24 & 22619 & 283.4 & -1.2 & 2.5 & 0.98 & 0.98 \\
& May 30 & 20879 & 293.5 & -0.7 & 2.3 & 0.87 & 0.92 \\
& May 31 & 21278 & 292.3 & -0.2 & 3.8 & 0.75 & 0.85 \\
& June 02 & 22259 & 287.1 & 0.8 & 2.8 & 0.71 & 0.82 \\
& June 03 & 24179 & 290.8 & 0.0 & 4.0 & 0.70 & 0.77 \\
& June 14 & 26459 & 289.0 & -3.5 & 4.5 & 0.97 & 0.97 \\
& June 16 & 24899 & 289.7 & -3.3 & 4.8 & 0.84 & 0.85 \\
& June 18 & 25499 & 288.6 & -2.0 & 3.6 & 0.88 & 0.91 \\
& June 20 & 25619 & 290.6 & -3.2 & 4.9 & 0.70 & 0.76 \\
& June 22 & 22679 & 287.3 & -9.6 & 10.1 & 0.92 & 0.74 \\
\hline
\end{tabular}


Table S6. Performance of simulated relative humidity (RH) in terms of bias, RMSE, R, and IA for the individual WP-3D aircraft flight paths.

\begin{tabular}{lccccccc}
\hline \multirow{2}{*}{ Variable } & $\begin{array}{c}\text { Flight } \\
\text { Date }\end{array}$ & $\begin{array}{c}\text { Number of } \\
\text { Data Points }\end{array}$ & $\begin{array}{c}\text { Observed } \\
\text { Mean }\end{array}$ & Bias & RMSE & R & IA \\
\hline \multirow{6}{*}{ May 04 } & 17219 & 22.5 & -7.4 & 13.6 & 0.72 & 0.73 \\
& May 07 & 24839 & 25.8 & 4.3 & 12.1 & 0.18 & 0.49 \\
& May 08 & 25439 & 30.2 & -0.9 & 15.1 & 0.72 & 0.83 \\
& May 11 & 25799 & 39.4 & 6.5 & 19.2 & 0.67 & 0.79 \\
& May 12 & 27489 & 41.6 & 1.4 & 21.4 & 0.51 & 0.71 \\
& May 14 & 22259 & 49.3 & -10.6 & 24.8 & 0.50 & 0.61 \\
& May 16 & 27899 & 51.9 & -6.2 & 19.4 & 0.81 & 0.89 \\
& May 19 & 24239 & 44.2 & -14.6 & 21.3 & 0.58 & 0.64 \\
& May 21 & 10722 & 40.5 & -6.4 & 17.5 & 0.83 & 0.90 \\
& May 24 & 22619 & 39.5 & -6.6 & 16.3 & 0.36 & 0.59 \\
& May 30 & 20879 & 21.7 & -4.0 & 11.7 & 0.78 & 0.76 \\
& May 31 & 21278 & 27.3 & -10.1 & 19.9 & 0.57 & 0.58 \\
& June 02 & 22259 & 59.6 & -12.2 & 20.2 & 0.41 & 0.57 \\
& June 03 & 24179 & 51.2 & -8.7 & 18.6 & 0.73 & 0.64 \\
& June 14 & 26459 & 30.1 & 5.3 & 13.8 & 0.70 & 0.82 \\
& June 16 & 24899 & 38.1 & -1.6 & 18.2 & 0.70 & 0.83 \\
& June 18 & 25499 & 41.5 & 0.3 & 14.7 & 0.84 & 0.92 \\
& June 20 & 25619 & 35.6 & -10.1 & 17.4 & 0.82 & 0.81 \\
& June 22 & 22679 & 16.8 & -0.7 & 8.8 & 0.18 & 0.47 \\
\hline
\end{tabular}


Table S7. Performance of wind speed (WS) in terms of bias, RMSE, R, and IA for the individual WP-3D aircraft flight paths.

\begin{tabular}{cccccccc}
\hline Variable & $\begin{array}{c}\text { Flight } \\
\text { Date }\end{array}$ & $\begin{array}{c}\text { Number of } \\
\text { Data Points }\end{array}$ & $\begin{array}{c}\text { Observed } \\
\text { Mean }\end{array}$ & Bias & RMSE & R & IA \\
\hline May 04 & 17219 & 4.9 & 1.5 & 3.3 & 0.35 & 0.61 \\
& May 07 & 24839 & 4.5 & 0.5 & 2.6 & 0.81 & 0.89 \\
& May 08 & 25439 & 7.9 & 0.1 & 3.6 & 0.76 & 0.87 \\
& May 11 & 25799 & 11.0 & 2.3 & 7.6 & 0.85 & 0.86 \\
& May 12 & 25289 & 7.3 & 0.3 & 6.3 & 0.49 & 0.62 \\
& May 14 & 22259 & 3.1 & -0.3 & 2.3 & 0.23 & 0.55 \\
& May 16 & 27899 & 5.1 & -1.7 & 3.1 & 0.44 & 0.60 \\
& May 19 & 24239 & 8.4 & -1.4 & 4.8 & 0.67 & 0.79 \\
& May 21 & 10722 & 10.4 & 1.6 & 3.9 & 0.83 & 0.90 \\
& May 24 & 22619 & 6.2 & -1.7 & 3.1 & 0.61 & 0.70 \\
May 30 & 20879 & 5.5 & 0.2 & 3.6 & 0.11 & 0.45 \\
& May 31 & 21278 & 4.1 & 1.1 & 3.8 & 0.11 & 0.48 \\
& June 02 & 22259 & 3.4 & 1.1 & 3.7 & 0.13 & 0.47 \\
& June 03 & 24179 & 3.3 & 0.5 & 2.7 & 0.39 & 0.62 \\
& June 14 & 26459 & 7.4 & -1.7 & 3.3 & 0.73 & 0.81 \\
June 16 & 24899 & 7.6 & -0.3 & 3.1 & 0.79 & 0.88 \\
June 18 & 25499 & 7.8 & -1.8 & 3.8 & 0.75 & 0.83 \\
June 20 & 25619 & 4.8 & 0.4 & 2.6 & 0.64 & 0.79 \\
June 22 & 22679 & 6.5 & -1.9 & 3.8 & 0.70 & 0.77 \\
June 20 & 25619 & 255.0 & 24.4 & 70.5 & 0.34 & 0.61 \\
June 22 & 22679 & 225.0 & 14.4 & 65.7 & 0.30 & 0.78 \\
\hline
\end{tabular}


Table S8. Performance of wind direction (WD) in terms of bias, RMSE, R, and IA for the individual WP-3D aircraft flight paths.

\begin{tabular}{cccccccc}
\hline \multirow{2}{*}{ Variable } & $\begin{array}{c}\text { Flight } \\
\text { Date }\end{array}$ & $\begin{array}{c}\text { Number of } \\
\text { Data Points }\end{array}$ & $\begin{array}{c}\text { Observed } \\
\text { Mean }\end{array}$ & Bias & RMSE & R & IA \\
\hline & May 04 & 17219 & 285.0 & 7.5 & 53.1 & 0.09 & 0.57 \\
& May 07 & 24839 & 345.0 & -10.3 & 76.4 & 0.16 & 0.81 \\
& May 08 & 25439 & 255.0 & -1.7 & 53.4 & 0.20 & 0.49 \\
& May 11 & 25799 & 345.0 & 1.3 & 40.1 & 0.11 & 0.85 \\
& May 12 & 25289 & 315.0 & -2.4 & 62.4 & 0.04 & 0.48 \\
& May 14 & 22259 & 255.0 & 20.6 & 99.2 & -0.11 & 0.73 \\
& May 16 & 27899 & 285.0 & -24.6 & 95.2 & -0.02 & 0.57 \\
WD $\left(^{\circ}\right)$ & May 19 & 24239 & 345.0 & 1.9 & 74.8 & 0.29 & 0.68 \\
& May 21 & 10722 & 315.0 & -1.1 & 35.0 & 0.26 & 0.52 \\
& May 24 & 22619 & 315.0 & -24.1 & 38.0 & 0.34 & 0.64 \\
& May 30 & 20879 & 285.0 & -2.1 & 66.1 & 0.42 & 0.90 \\
& May 31 & 21278 & 315.0 & -18.1 & 87.3 & 0.12 & 0.70 \\
& June 02 & 22259 & 285.0 & -11.8 & 84.2 & 0.23 & 0.84 \\
& June 03 & 24179 & 345.0 & -10.1 & 84.3 & 0.17 & 0.84 \\
& June 14 & 26459 & 255.0 & -16.8 & 57.6 & 0.51 & 0.84 \\
& June 16 & 24899 & 315.0 & -7.5 & 39.2 & 0.28 & 0.80 \\
& June 18 & 25499 & 255.0 & -3.2 & 47.0 & 0.57 & 0.83 \\
\hline
\end{tabular}


Table S9. Performance of simulated temperature (T) in terms of bias, RMSE, R, and IA for the individual CIRPAS Twin Otter aircraft flight paths.

\begin{tabular}{lccccccc}
\hline \multirow{2}{*}{ Variable } & $\begin{array}{c}\text { Flight } \\
\text { Date }\end{array}$ & $\begin{array}{c}\text { Number of } \\
\text { Data Points }\end{array}$ & $\begin{array}{c}\text { Observed } \\
\text { Mean }\end{array}$ & Bias & RMSE & R & IA \\
\hline \multirow{5}{*}{ May 04 } & 187 & 293.7 & -2.3 & 3.5 & 0.14 & 0.40 \\
& May 05 & 228 & 291.8 & -2.0 & 3.6 & -0.09 & 0.33 \\
& May 06 & 179 & 290.0 & -2.0 & 2.6 & 0.17 & 0.46 \\
& May 07 & 194 & 294.9 & -3.6 & 5.0 & 0.09 & 0.32 \\
& May 10 & 182 & 286.7 & -1.1 & 2.4 & 0.48 & 0.54 \\
& May 12 & 195 & 291.9 & -4.8 & 5.5 & 0.67 & 0.54 \\
& May 13 & 174 & 294.3 & -2.3 & 3.6 & 0.76 & 0.80 \\
& May 14 & 189 & 289.5 & 0.4 & 1.5 & 0.68 & 0.77 \\
& May 15 & 185 & 292.2 & -1.7 & 2.7 & 0.72 & 0.74 \\
& May 18 & 183 & 283.6 & -2.5 & 3.5 & 0.96 & 0.93 \\
& May 19 & 192 & 290.6 & -1.1 & 2.1 & 0.37 & 0.54 \\
& May 20 & 192 & 288.0 & -4.6 & 5.3 & 0.85 & 0.74 \\
& May 21 & 192 & 288.7 & -2.4 & 3.0 & 0.58 & 0.63 \\
& May 22 & 195 & 279.1 & -4.3 & 4.7 & 0.95 & 0.87 \\
& May 24 & 189 & 285.8 & -5.7 & 6.7 & 0.87 & 0.76 \\
& May 25 & 191 & 289.8 & -5.7 & 6.5 & 0.81 & 0.63 \\
& May 27 & 176 & 287.9 & -3.4 & 4.1 & 0.28 & 0.32 \\
& May 28 & 192 & 288.1 & -4.2 & 4.7 & 0.38 & 0.27 \\
\hline
\end{tabular}


Table S10. Performance of simulated relative humidity (RH) in terms of bias, RMSE, R, and IA for the individual CIRPAS Twin Otter aircraft flight paths.

\begin{tabular}{lccccccc}
\hline \multirow{2}{*}{ Variable } & $\begin{array}{c}\text { Flight } \\
\text { Date }\end{array}$ & $\begin{array}{c}\text { Number of } \\
\text { Data Points }\end{array}$ & $\begin{array}{c}\text { Observed } \\
\text { Mean }\end{array}$ & Bias & RMSE & R & IA \\
\hline & May 04 & 187 & 40.1 & -10.1 & 12.1 & 0.87 & 0.78 \\
& May 05 & 228 & 51.9 & -16.2 & 19.0 & 0.78 & 0.67 \\
& May 06 & 178 & 57.4 & -17.2 & 41.4 & 0.29 & 0.37 \\
& May 07 & 194 & 36.0 & -9.3 & 20.8 & 0.57 & 0.61 \\
& May 10 & 182 & 61.6 & 4.8 & 13.0 & -0.12 & 0.27 \\
& May 12 & 195 & 35.9 & 6.7 & 11.7 & 0.85 & 0.88 \\
& May 13 & 174 & 31.8 & -3.6 & 10.1 & 0.79 & 0.82 \\
& May 14 & 189 & 65.1 & -18.2 & 22.2 & 0.67 & 0.55 \\
& May 15 & 185 & 57.3 & -12.7 & 18.3 & 0.71 & 0.75 \\
& May 18 & 183 & 48.5 & -1.8 & 14.6 & 0.90 & 0.90 \\
& May 19 & 192 & 64.8 & -18.2 & 21.9 & 0.62 & 0.51 \\
& May 20 & 192 & 38.9 & 6.4 & 22.5 & 0.78 & 0.82 \\
& May 21 & 192 & 64.3 & -18.5 & 26.4 & 0.56 & 0.63 \\
& May 22 & 194 & 38.2 & 1.7 & 25.9 & 0.46 & 0.68 \\
& May 24 & 189 & 35.9 & 4.2 & 13.0 & 0.73 & 0.85 \\
& May 25 & 191 & 38.5 & 0.1 & 11.1 & 0.72 & 0.85 \\
& May 27 & 176 & 66.1 & 7.3 & 15.6 & -0.29 & 0.13 \\
& May 28 & 192 & 54.9 & -6.9 & 12.4 & 0.68 & 0.68 \\
\hline
\end{tabular}


Table S11. Performance of simulated temperature (T) in terms of bias, RMSE, R, and IA for the $\mathrm{R} / \mathrm{V}$ Atlantis paths by day.

\begin{tabular}{cccccccc}
\hline \multirow{2}{*}{ Variable } & $\begin{array}{c}\text { Flight } \\
\text { Date }\end{array}$ & $\begin{array}{c}\text { Number of } \\
\text { Data Points }\end{array}$ & $\begin{array}{c}\text { Observed } \\
\text { Mean }\end{array}$ & Bias & RMSE & R & IA \\
\hline May 14 & 359 & 287.6 & -1.9 & 2.0 & 0.03 & 0.17 \\
May 15 & 1409 & 286.6 & 0.6 & 1.3 & -0.43 & 0.11 \\
May 16 & 1424 & 285.9 & 1.6 & 1.7 & 0.25 & 0.35 \\
& May 17 & 1433 & 285.0 & 0.8 & 1.0 & 0.12 & 0.46 \\
& May 18 & 1413 & 285.7 & 0.7 & 1.0 & 0.35 & 0.53 \\
May 19 & 1427 & 286.8 & 0.5 & 0.9 & -0.57 & 0.31 \\
May 20 & 1439 & 288.0 & -0.1 & 1.9 & 0.43 & 0.62 \\
May 21 & 1433 & 288.1 & 1.6 & 2.7 & -0.23 & 0.12 \\
May 22 & 1417 & 288.4 & 1.5 & 2.1 & 0.12 & 0.47 \\
May 23 & 1440 & 286.1 & 1.1 & 1.3 & 0.48 & 0.51 \\
May 24 & 1429 & 286.2 & 0.7 & 0.9 & -0.07 & 0.44 \\
May 25 & 1440 & 286.0 & 0.9 & 1.2 & -0.17 & 0.44 \\
May 26 & 1440 & 288.2 & -0.5 & 1.1 & 0.90 & 0.89 \\
May 27 & 1440 & 288.6 & 0.4 & 1.4 & 0.51 & 0.70 \\
May 28 & 1440 & 287.8 & 0.8 & 1.5 & 0.46 & 0.59 \\
May 29 & 1440 & 287.5 & 0.6 & 1.4 & 0.24 & 0.45 \\
May 30 & 1434 & 288.9 & 1.6 & 3.3 & 0.11 & 0.34 \\
May 31 & 1432 & 287.1 & 3.7 & 4.8 & -0.18 & 0.12 \\
June 01 & 1440 & 285.1 & 1.6 & 1.7 & 0.67 & 0.45 \\
June 02 & 1440 & 285.0 & 0.5 & 1.4 & 0.29 & 0.48 \\
June 03 & 1440 & 291.2 & 3.8 & 4.5 & 0.79 & 0.74 \\
June 04 & 1440 & 293.4 & 0.2 & 2.8 & -0.30 & 0.12 \\
June 05 & 1440 & 294.1 & -2.3 & 5.2 & -0.66 & 0.31 \\
June 06 & 1440 & 290.6 & 5.1 & 6.7 & 0.66 & 0.55 \\
June 07 & 1440 & 285.9 & 0.6 & 1.3 & 0.87 & 0.86 \\
June 08 & 720 & 285.8 & 1.8 & 2.1 & 0.84 & 0.41 \\
\hline
\end{tabular}


Table S12. Performance of simulated relative humidity (RH) in terms of bias, RMSE, R, and IA for the R/V Atlantis paths by day.

\begin{tabular}{cccccccc}
\hline \multirow{2}{*}{ Variable } & $\begin{array}{c}\text { Flight } \\
\text { Date }\end{array}$ & $\begin{array}{c}\text { Number of } \\
\text { Data Points }\end{array}$ & $\begin{array}{c}\text { Observed } \\
\text { Mean }\end{array}$ & Bias & RMSE & R & IA \\
\hline May 14 & 359 & 79.7 & 14.5 & 14.6 & 0.14 & 0.12 \\
& May 15 & 1409 & 88.2 & 2.4 & 6.4 & -0.07 & 0.33 \\
& May 16 & 1424 & 92.8 & -2.3 & 3.8 & -0.05 & 0.40 \\
& May 17 & 1433 & 94.7 & -2.3 & 4.4 & 0.46 & 0.56 \\
& May 18 & 1413 & 94.8 & -1.6 & 3.0 & 0.06 & 0.42 \\
& May 19 & 1427 & 94.3 & -3.8 & 4.7 & -0.11 & 0.37 \\
& May 20 & 1439 & 91.4 & -4.3 & 13.4 & 0.35 & 0.46 \\
& May 21 & 1433 & 83.5 & -13.2 & 17.7 & -0.11 & 0.21 \\
& May 22 & 1417 & 71.3 & -7.8 & 11.5 & 0.27 & 0.53 \\
& May 23 & 1440 & 67.5 & 3.6 & 5.4 & 0.84 & 0.86 \\
May 24 & 1429 & 80.0 & -3.4 & 4.7 & 0.58 & 0.57 \\
May 25 & 1440 & 85.0 & -9.2 & 12.5 & -0.52 & 0.20 \\
May 26 & 1440 & 81.2 & 2.1 & 5.8 & 0.90 & 0.94 \\
May 27 & 1440 & 76.2 & -1.7 & 9.6 & 0.63 & 0.79 \\
May 28 & 1440 & 78.4 & -13.8 & 16.8 & 0.67 & 0.51 \\
May 29 & 1440 & 84.4 & -10.3 & 13.9 & 0.03 & 0.30 \\
May 30 & 1434 & 86.4 & -20.6 & 28.3 & 0.22 & 0.46 \\
May 31 & 1432 & 91.7 & -29.9 & 33.3 & -0.13 & 0.09 \\
June 01 & 1440 & 95.9 & -3.2 & 8.8 & 0.14 & 0.35 \\
June 02 & 1440 & 93.1 & -8.8 & 11.6 & 0.41 & 0.53 \\
June 03 & 1440 & 79.1 & -9.0 & 14.6 & 0.71 & 0.74 \\
June 04 & 1440 & 71.3 & -3.5 & 14.6 & -0.11 & 0.29 \\
June 05 & 1440 & 74.0 & 6.3 & 23.2 & -0.68 & 0.20 \\
June 06 & 1440 & 84.1 & -28.0 & 32.2 & 0.59 & 0.51 \\
June 07 & 1440 & 95.6 & 1.5 & 2.8 & 0.95 & 0.97 \\
June 08 & 720 & 89.2 & -10.3 & 11.2 & 0.91 & 0.37 \\
\hline
\end{tabular}


Table S13. Performance of simulated wind speed (WS) in terms of bias, RMSE, R, and IA for the R/V Atlantis paths by day.

\begin{tabular}{cccccccc}
\hline \multirow{2}{*}{ Variable } & $\begin{array}{c}\text { Flight } \\
\text { Date }\end{array}$ & $\begin{array}{c}\text { Number of } \\
\text { Data Points }\end{array}$ & $\begin{array}{c}\text { Observed } \\
\text { Mean }\end{array}$ & Bias & RMSE & R & IA \\
\hline May 14 & 359 & 4.4 & -1.0 & 1.3 & 0.42 & 0.48 \\
& May 15 & 1408 & 3.3 & 0.4 & 2.0 & 0.15 & 0.41 \\
& May 16 & 1424 & 3.6 & 1.8 & 2.6 & 0.46 & 0.56 \\
& May 17 & 1433 & 2.8 & 0.2 & 2.6 & -0.31 & 0.23 \\
& May 18 & 1413 & 4.4 & -2.7 & 3.6 & 0.45 & 0.50 \\
& May 19 & 1427 & 5.2 & 1.0 & 2.6 & 0.01 & 0.43 \\
& May 20 & 1439 & 4.3 & 1.4 & 2.6 & 0.02 & 0.43 \\
May 21 & 1433 & 4.5 & 4.4 & 5.8 & -0.05 & 0.34 \\
& May 22 & 1417 & 2.8 & 4.4 & 4.9 & 0.84 & 0.53 \\
& May 23 & 1440 & 9.6 & 2.5 & 4.1 & 0.34 & 0.49 \\
May 24 & 1429 & 5.9 & 7.8 & 8.8 & -0.71 & 0.34 \\
& May 25 & 1440 & 4.7 & 3.8 & 5.6 & -0.07 & 0.40 \\
May 26 & 1440 & 4.4 & 3.0 & 3.6 & 0.26 & 0.47 \\
& May 27 & 1440 & 2.6 & 3.1 & 3.4 & -0.28 & 0.33 \\
& May 28 & 1440 & 5.4 & 3.8 & 4.5 & 0.31 & 0.46 \\
May 29 & 1440 & 3.7 & 6.1 & 6.7 & -0.22 & 0.28 \\
May 30 & 1434 & 4.3 & 3.5 & 4.5 & 0.14 & 0.45 \\
May 31 & 1432 & 2.9 & 0.5 & 3.2 & 0.35 & 0.41 \\
June 01 & 1440 & 7.9 & 2.0 & 4.3 & 0.41 & 0.63 \\
June 02 & 1440 & 4.4 & 6.3 & 8.5 & -0.20 & 0.35 \\
June 03 & 1440 & 5.6 & 0.8 & 2.3 & 0.28 & 0.49 \\
June 04 & 1440 & 4.8 & -0.5 & 1.5 & 0.12 & 0.33 \\
June 05 & 1440 & 3.3 & -1.8 & 2.4 & 0.84 & 0.59 \\
June 06 & 1440 & 6.5 & -0.5 & 2.1 & 0.01 & 0.38 \\
June 07 & 1440 & 10.9 & -2.2 & 3.4 & 0.47 & 0.56 \\
June 08 & 720 & 5.7 & 3.5 & 4.1 & 0.35 & 0.41 \\
\hline & & & & & & &
\end{tabular}


Table S14. Performance of simulated wind direction (WD) in terms of bias, RMSE, R, and IA for the R/V Atlantis paths by day.

\begin{tabular}{|c|c|c|c|c|c|c|c|}
\hline Variable & $\begin{array}{l}\text { Flight } \\
\text { Date }\end{array}$ & $\begin{array}{l}\text { Number of } \\
\text { Data Points }\end{array}$ & $\begin{array}{c}\text { Observed } \\
\text { Mean }\end{array}$ & Bias & RMSE & $\mathbf{R}$ & IA \\
\hline \multirow{26}{*}{$\mathrm{WD}\left(^{\circ}\right)$} & May 14 & 359 & 225.0 & -9.0 & 29.7 & -0.45 & 0.21 \\
\hline & May 15 & 1408 & 255.0 & 0.4 & 65.5 & -0.09 & 0.43 \\
\hline & May 16 & 1424 & 195.0 & 41.0 & 90.4 & 0.25 & 0.52 \\
\hline & May 17 & 1433 & 255.0 & -40.7 & 99.6 & -0.36 & 0.20 \\
\hline & May 18 & 1413 & 105.0 & 65.4 & 129.3 & 0.46 & 0.54 \\
\hline & May 19 & 1427 & 255.0 & 19.1 & 29.2 & 0.47 & 0.51 \\
\hline & May 20 & 1439 & 225.0 & 51.0 & 73.3 & 0.22 & 0.52 \\
\hline & May 21 & 1433 & 225.0 & -9.3 & 71.1 & 0.43 & 0.60 \\
\hline & May 22 & 1417 & 255.0 & 22.9 & 55.4 & 0.20 & 0.44 \\
\hline & May 23 & 1440 & 285.0 & 5.2 & 16.8 & 0.32 & 0.51 \\
\hline & May 24 & 1429 & 255.0 & 16.2 & 104.1 & 0.82 & 0.63 \\
\hline & May 25 & 1440 & 285.0 & 16.7 & 80.7 & 0.43 & 0.55 \\
\hline & May 26 & 1440 & 285.0 & 17.0 & 57.4 & -0.25 & 0.45 \\
\hline & May 27 & 1440 & 285.0 & 9.9 & 87.1 & 0.26 & 0.52 \\
\hline & May 28 & 1440 & 285.0 & 13.0 & 63.0 & -0.41 & 0.41 \\
\hline & May 29 & 1440 & 255.0 & -17.6 & 82.6 & -0.50 & 0.73 \\
\hline & May 30 & 1434 & 105.0 & -18.7 & 118.2 & -0.20 & 0.60 \\
\hline & May 31 & 1432 & 315.0 & 32.6 & 91.8 & 0.46 & 0.58 \\
\hline & June 01 & 1440 & 315.0 & -7.4 & 39.1 & 0.67 & 0.72 \\
\hline & June 02 & 1440 & 285.0 & 19.3 & 77.1 & 0.16 & 0.53 \\
\hline & June 03 & 1440 & 225.0 & 18.5 & 35.8 & 0.19 & 0.48 \\
\hline & June 04 & 1440 & 195.0 & 18.6 & 32.1 & 0.24 & 0.45 \\
\hline & June 05 & 1440 & 135.0 & -5.5 & 54.6 & 0.52 & 0.70 \\
\hline & June 06 & 1440 & 285.0 & 14.9 & 25.6 & 0.80 & 0.78 \\
\hline & June 07 & 1440 & 315.0 & 11.4 & 24.3 & 0.89 & 0.77 \\
\hline & June 08 & 720 & 225.0 & 31.9 & 35.7 & -0.03 & 0.38 \\
\hline
\end{tabular}


Table S15. Performance of simulated carbon monoxide (CO), the sum of nitrogen oxide and nitrogen dioxide $\left(\mathrm{NO}_{\mathrm{x}}\right)$, sulfur dioxide $\left(\mathrm{SO}_{2}\right)$, and ozone $\left(\mathrm{O}_{3}\right)$, from the DEF_ANT simulation in terms of bias, RMSE, R, and IA relative to observations at the four supersites (Fig. 1a,b) and the surface operational air quality sampling stations depicted in Fig. 1d. Statistics given for all of California (CA) and by regions as shown in Fig. 1c. 
Table S15. (continued).

\begin{tabular}{|c|c|c|c|c|c|c|}
\hline Variables & $\begin{array}{c}\text { Regions and } \\
\text { Supersites }\end{array}$ & $\begin{array}{c}\text { Observed } \\
\text { Mean }\end{array}$ & Bias & RMSE & $\mathbf{R}$ & IA \\
\hline \multirow{10}{*}{$\mathrm{CO}(\mathrm{ppbv})$} & $\mathrm{CA}$ & 269.9 & -2.0 & 260.0 & 0.14 & 0.39 \\
\hline & Southern CA & 319.8 & 1.3 & 298.4 & 0.03 & 0.35 \\
\hline & San Joaquin & 172.9 & 31.2 & 115.2 & 0.20 & 0.45 \\
\hline & Sacramento Valley & 201.6 & 4.8 & 98.0 & 0.20 & 0.45 \\
\hline & Coastal & 206.2 & -25.0 & 143.2 & 0.24 & 0.48 \\
\hline & Interior Mountains & 249.0 & -59.3 & 424.0 & 0.07 & 0.11 \\
\hline & Bakersfield & 166.7 & 48.1 & 81.3 & 0.47 & 0.50 \\
\hline & Pasadena & 306.6 & 225.6 & 291.9 & 0.45 & 0.36 \\
\hline & T0 & 154.5 & 79.5 & 129.1 & 0.23 & 0.38 \\
\hline & $\mathrm{T} 1$ & 122.8 & 69.1 & 86.0 & 0.31 & 0.33 \\
\hline \multirow{10}{*}{$\mathrm{NO}_{\mathrm{x}}(\mathrm{ppbv})$} & $\mathrm{CA}$ & 9.7 & 6.2 & 22.4 & 0.33 & 0.46 \\
\hline & Southern CA & 13.1 & 9.1 & 29.7 & 0.24 & 0.40 \\
\hline & San Joaquin & 8.3 & 5.8 & 15.8 & 0.52 & 0.55 \\
\hline & Sacramento Valley & 6.2 & 6.5 & 13.1 & 0.36 & 0.42 \\
\hline & Coastal & 4.5 & 2.4 & 8.9 & 0.36 & 0.52 \\
\hline & Interior Mountains & 8.5 & -2.0 & 10.8 & 0.23 & 0.43 \\
\hline & Bakersfield & - & - & - & - & - \\
\hline & Pasadena & 16.3 & 40.8 & 50.5 & 0.45 & 0.27 \\
\hline & T0 & 7.0 & 8.3 & 14.7 & 0.25 & 0.34 \\
\hline & $\mathrm{T} 1$ & - & - & - & - & - \\
\hline \multirow{10}{*}{$\mathrm{SO}_{2}(\mathrm{ppbv})$} & $\mathrm{CA}$ & 0.8 & 0.9 & 3.5 & 0.05 & 0.17 \\
\hline & Southern CA & 0.9 & 1.4 & 4.4 & 0.03 & 0.14 \\
\hline & San Joaquin & 1.3 & -0.3 & 1.1 & 0.14 & 0.45 \\
\hline & Sacramento Valley & 0.7 & 0.3 & 1.2 & 0.47 & 0.64 \\
\hline & Coastal & 0.4 & 0.7 & 2.5 & -0.07 & 0.12 \\
\hline & Interior Mountains & 0.9 & -0.5 & 1.7 & 0.04 & 0.30 \\
\hline & Bakersfield & 0.8 & 0.0 & 1.0 & -0.21 & 0.24 \\
\hline & Pasadena & 0.3 & 1.4 & 2.0 & 0.05 & 0.14 \\
\hline & T0 & 0.4 & 0.4 & 0.9 & 0.32 & 0.47 \\
\hline & $\mathrm{T} 1$ & - & - & - & - & - \\
\hline \multirow{10}{*}{$\mathrm{O}_{3}(\mathrm{ppbv})$} & $\mathrm{CA}$ & 36.6 & -3.9 & 13.9 & 0.71 & 0.83 \\
\hline & Southern CA & 38.5 & -2.8 & 15.9 & 0.65 & 0.80 \\
\hline & San Joaquin & 38.7 & -8.6 & 14.0 & 0.84 & 0.86 \\
\hline & Sacramento Valley & 30.4 & -6.1 & 12.5 & 0.77 & 0.83 \\
\hline & Coastal & 29.5 & -2.2 & 11.4 & 0.61 & 0.77 \\
\hline & Interior Mountains & 42.4 & -3.7 & 11.8 & 0.73 & 0.84 \\
\hline & Bakersfield & 39.1 & -13.2 & 17.8 & 0.84 & 0.83 \\
\hline & Pasadena & 33.1 & -14.8 & 20.4 & 0.68 & 0.70 \\
\hline & T0 & 32.1 & -8.0 & 13.5 & 0.86 & 0.87 \\
\hline & $\mathrm{T} 1$ & 39.7 & -5.4 & 13.0 & 0.76 & 0.84 \\
\hline
\end{tabular}


Table S16. Same as Table S15, except from 50\%_ANT simulation.

\begin{tabular}{|c|c|c|c|c|c|c|}
\hline Variable & $\begin{array}{l}\text { Regions and } \\
\text { Supersites }\end{array}$ & $\begin{array}{c}\text { Observed } \\
\text { Mean }\end{array}$ & Bias & RMSE & $\mathbf{R}$ & $\mathbf{I A}$ \\
\hline \multirow{10}{*}{$\mathrm{CO}$ (ppbv) } & $\mathrm{CA}$ & 269.9 & -71.9 & 243.7 & 0.17 & 0.37 \\
\hline & Southern CA & 319.8 & -92.5 & 273.5 & 0.07 & 0.38 \\
\hline & San Joaquin & 172.9 & -10.3 & 106.0 & 0.20 & 0.35 \\
\hline & Sacramento Valley & 201.6 & -37.3 & 89.4 & 0.21 & 0.44 \\
\hline & Coastal & 206.2 & -56.6 & 146.6 & 0.25 & 0.45 \\
\hline & Interior Mountains & 249.0 & -92.9 & 429.3 & 0.05 & 0.13 \\
\hline & Bakersfield & 166.7 & -0.9 & 39.8 & 0.49 & 0.69 \\
\hline & Pasadena & 306.6 & 24.6 & 105.6 & 0.43 & 0.63 \\
\hline & T0 & 154.5 & 18.9 & 72.0 & 0.26 & 0.46 \\
\hline & $\mathrm{T} 1$ & 122.8 & 32.1 & 45.5 & 0.27 & 0.46 \\
\hline \multirow{10}{*}{$\mathrm{NO}_{\mathrm{x}}(\mathrm{ppbv})$} & $\mathrm{CA}$ & 9.74 & -2.05 & 13.4 & 0.33 & 0.56 \\
\hline & Southern CA & 13.1 & -2.45 & 17.6 & 0.24 & 0.51 \\
\hline & San Joaquin & 8.30 & -1.42 & 8.1 & 0.53 & 0.70 \\
\hline & Sacramento Valley & 6.16 & -0.02 & 6.5 & 0.37 & 0.58 \\
\hline & Coastal & 4.51 & -1.09 & 6.0 & 0.36 & 0.57 \\
\hline & Interior Mountains & 8.49 & -5.41 & 11.4 & 0.24 & 0.36 \\
\hline & Bakersfield & - & - & - & - & - \\
\hline & Pasadena & 16.3 & 11.03 & 17.9 & 0.47 & 0.53 \\
\hline & $\mathrm{T} 0$ & 7.00 & 0.36 & 7.3 & 0.26 & 0.47 \\
\hline & $\mathrm{T} 1$ & - & - & - & - & - \\
\hline \multirow{10}{*}{$\mathrm{SO}_{2}(\mathrm{ppbv})$} & $\mathrm{CA}$ & 0.77 & 0.88 & 3.5 & 0.05 & 0.17 \\
\hline & Southern CA & 0.88 & 1.35 & 4.4 & 0.03 & 0.14 \\
\hline & San Joaquin & 1.28 & -0.34 & 1.1 & 0.15 & 0.46 \\
\hline & Sacramento Valley & 0.72 & 0.30 & 1.2 & 0.46 & 0.64 \\
\hline & Coastal & 0.41 & 0.71 & 2.5 & -0.07 & 0.13 \\
\hline & Interior Mountains & 0.86 & -0.53 & 1.7 & 0.04 & 0.30 \\
\hline & Bakersfield & 0.79 & 0.00 & 1.0 & -0.21 & 0.24 \\
\hline & Pasadena & 0.32 & 1.34 & 2.0 & 0.05 & 0.14 \\
\hline & T0 & 0.38 & 0.42 & 0.9 & 0.33 & 0.48 \\
\hline & $\mathrm{T} 1$ & - & - & - & - & - \\
\hline \multirow{10}{*}{$\mathrm{O}_{3}(\mathrm{ppbv})$} & $\mathrm{CA}$ & 36.6 & -4.0 & 13.1 & 0.70 & 0.81 \\
\hline & Southern CA & 38.5 & -1.7 & 14.9 & 0.63 & 0.79 \\
\hline & San Joaquin & 38.7 & -8.9 & 13.0 & 0.83 & 0.85 \\
\hline & Sacramento Valley & 30.4 & -5.5 & 10.7 & 0.78 & 0.84 \\
\hline & Coastal & 29.5 & -2.6 & 10.5 & 0.61 & 0.77 \\
\hline & Interior Mountains & 42.4 & -6.8 & 12.4 & 0.73 & 0.79 \\
\hline & Bakersfield & 39.1 & -12.2 & 16.0 & 0.85 & 0.83 \\
\hline & Pasadena & 33.1 & -8.4 & 16.9 & 0.66 & 0.77 \\
\hline & $\mathrm{T} 0$ & 32.1 & -7.0 & 11.6 & 0.85 & 0.88 \\
\hline & $\mathrm{T} 1$ & 39.7 & -9.1 & 13.2 & 0.77 & 0.80 \\
\hline
\end{tabular}


Table S17. Same as Table S15, except from 0\%_ANT simulation.

\begin{tabular}{|c|c|c|c|c|c|c|}
\hline Variable & $\begin{array}{c}\text { Regions and } \\
\text { Supersites }\end{array}$ & $\begin{array}{c}\text { Observed } \\
\text { Mean }\end{array}$ & Bias & RMSE & $\mathbf{R}$ & IA \\
\hline \multirow{10}{*}{$\mathrm{CO}$ (ppbv) } & $\mathrm{CA}$ & 269.9 & -140.2 & 268.2 & 0.19 & 0.37 \\
\hline & Southern CA & 319.8 & -183.9 & 304.8 & 0.17 & 0.40 \\
\hline & San Joaquin & 172.9 & -51.3 & 118.1 & 0.13 & 0.39 \\
\hline & Sacramento Valley & 201.6 & -79.0 & 112.7 & 0.05 & 0.39 \\
\hline & Coastal & 206.2 & -87.5 & 164.0 & 0.11 & 0.42 \\
\hline & Interior Mountains & 249.0 & -125.7 & 438.7 & -0.03 & 0.15 \\
\hline & Bakersfield & 166.7 & -49.6 & 62.8 & 0.27 & 0.43 \\
\hline & Pasadena & 306.6 & -171.9 & 194.8 & -0.05 & 0.37 \\
\hline & T0 & 154.5 & -41.3 & 74.7 & 0.33 & 0.39 \\
\hline & $\mathrm{T} 1$ & 122.8 & -5.1 & 28.3 & -0.16 & 0.26 \\
\hline \multirow{10}{*}{$\mathrm{NO}_{\mathrm{x}}(\mathrm{ppbv})$} & $\mathrm{CA}$ & 9.74 & -9.57 & 15.2 & 0.18 & 0.38 \\
\hline & Southern CA & 13.1 & -12.94 & 19.4 & 0.13 & 0.39 \\
\hline & San Joaquin & 8.30 & -8.11 & 11.2 & 0.39 & 0.39 \\
\hline & Sacramento Valley & 6.16 & -5.96 & 8.2 & 0.32 & 0.39 \\
\hline & Coastal & 4.51 & -4.42 & 7.3 & 0.21 & 0.38 \\
\hline & Interior Mountains & 8.49 & -8.36 & 13.3 & 0.06 & 0.36 \\
\hline & Bakersfield & - & - & - & - & - \\
\hline & Pasadena & 16.3 & -16.1 & 18.7 & -0.10 & 0.38 \\
\hline & T0 & 7.00 & -6.77 & 9.1 & 0.37 & 0.38 \\
\hline & $\mathrm{T} 1$ & - & - & - & - & - \\
\hline \multirow{10}{*}{$\mathrm{SO}_{2}(\mathrm{ppbv})$} & $\mathrm{CA}$ & 0.77 & -0.70 & 1.2 & 0.04 & 0.40 \\
\hline & Southern CA & 0.88 & -0.76 & 1.3 & 0.01 & 0.40 \\
\hline & San Joaquin & 1.28 & -1.28 & 1.5 & -0.04 & 0.39 \\
\hline & Sacramento Valley & 0.72 & -0.71 & 1.2 & -0.01 & 0.43 \\
\hline & Coastal & 0.41 & -0.41 & 0.7 & 0.01 & 0.47 \\
\hline & Interior Mountains & 0.86 & -0.85 & 1.8 & -0.02 & 0.35 \\
\hline & Bakersfield & 0.79 & -0.79 & 1.0 & -0.15 & 0.42 \\
\hline & Pasadena & 0.32 & -0.24 & 0.4 & -0.12 & 0.37 \\
\hline & T0 & 0.38 & -0.37 & 0.7 & 0.05 & 0.35 \\
\hline & $\mathrm{T} 1$ & - & - & - & - & - \\
\hline \multirow{10}{*}{$\mathrm{O}_{3}(\mathrm{ppbv})$} & $\mathrm{CA}$ & 36.6 & -9.8 & 18.6 & 0.39 & 0.57 \\
\hline & Southern CA & 38.5 & -8.1 & 20.7 & 0.24 & 0.50 \\
\hline & San Joaquin & 38.7 & -14.8 & 19.9 & 0.60 & 0.59 \\
\hline & Sacramento Valley & 30.4 & -8.2 & 13.5 & 0.60 & 0.68 \\
\hline & Coastal & 29.5 & -6.2 & 12.5 & 0.48 & 0.62 \\
\hline & Interior Mountains & 42.4 & -15.5 & 20.6 & 0.44 & 0.54 \\
\hline & Bakersfield & 39.1 & -16.4 & 22.6 & 0.67 & 0.59 \\
\hline & Pasadena & 33.1 & -5.4 & 20.2 & 0.26 & 0.53 \\
\hline & T0 & 32.1 & -12.2 & 17.3 & 0.67 & 0.63 \\
\hline & $\mathrm{T} 1$ & 39.7 & -19.9 & 23.5 & 0.52 & 0.51 \\
\hline
\end{tabular}


Table S18. Performance of simulated carbon monoxide (CO) from the DEF_ANT simulation in terms of bias, RMSE, R, and IA for each for the G-1 flights.

\begin{tabular}{cccccccc}
\hline \multirow{2}{*}{ Variable } & $\begin{array}{c}\text { Flight } \\
\text { Date }\end{array}$ & $\begin{array}{c}\text { Number of } \\
\text { Data Points }\end{array}$ & $\begin{array}{c}\text { Observed } \\
\text { Mean }\end{array}$ & Bias & RMSE & R & IA \\
\hline \multirow{7}{*}{ June 03a } & 800 & 129.7 & 0.8 & 14.8 & 0.12 & 0.44 \\
& June 06a & 1097 & 120.6 & 11.8 & 20.3 & 0.81 & 0.76 \\
& June 06b & 943 & 124.8 & -2.1 & 16.8 & 0.31 & 0.59 \\
& June 08a & 1142 & 156.1 & -7.1 & 20.2 & 0.54 & 0.71 \\
& June 08b & 1147 & 149.0 & 7.9 & 28.6 & 0.71 & 0.69 \\
& June 10a & 1068 & 126.3 & -5.0 & 9.3 & 0.18 & 0.43 \\
& June 12a & 1042 & 113.2 & 10.6 & 16.7 & 0.13 & 0.33 \\
& June 12b & 950 & 127.7 & 4.5 & 10.6 & 0.03 & 0.41 \\
& June 14a & 1120 & 164.7 & 5.3 & 35.1 & 0.36 & 0.60 \\
& June 15a & 1055 & 144.9 & 32.7 & 37.5 & 0.73 & 0.49 \\
& June 15b & 1071 & 150.5 & 25.3 & 35.1 & 0.29 & 0.44 \\
& June 18a & 1114 & 132.9 & -2.6 & 24.4 & 0.39 & 0.58 \\
& June 19a & 1050 & 135.3 & 27.6 & 31.6 & 0.41 & 0.36 \\
& June 21a & 1077 & 134.6 & 14.9 & 25.3 & 0.54 & 0.63 \\
& June 21b & 1043 & 128.0 & 18.0 & 26.0 & 0.39 & 0.48 \\
& June 23a & 1012 & 171.8 & 3.6 & 49.8 & -0.16 & 0.27 \\
& June 23b & 1003 & 157.3 & 27.6 & 42.0 & 0.24 & 0.42 \\
& June 24a & 1122 & 110.4 & 25.1 & 57.7 & -0.37 & 0.26 \\
& June 24b & 922 & 120.8 & 17.5 & 42.7 & 0.35 & 0.52 \\
& June 27a & 1163 & 147.4 & 42.6 & 62.2 & 0.36 & 0.49 \\
& June 28a & 1051 & 166.3 & 54.0 & 78.7 & 0.65 & 0.64 \\
& June 28b & 750 & 155.7 & 0.6 & 24.2 & 0.54 & 0.71 \\
\hline
\end{tabular}


Table S19. Performance of simulated nitrogen oxide (NO) from the DEF_ANT simulation in terms of bias, RMSE, R, and IA for each for the G-1 flights.

\begin{tabular}{cccccccc}
\hline \multirow{2}{*}{ Variable } & $\begin{array}{c}\text { Flight } \\
\text { Date }\end{array}$ & $\begin{array}{c}\text { Number of } \\
\text { Data Points }\end{array}$ & $\begin{array}{c}\text { Observed } \\
\text { Mean }\end{array}$ & Bias & RMSE & R & IA \\
\hline \multirow{6}{*}{ June 03a } & 816 & 0.3 & 0.4 & 0.9 & 0.59 & 0.52 \\
& June 06a & 1069 & 0.2 & 0.6 & 1.2 & 0.85 & 0.48 \\
& June 06b & 946 & 0.1 & 0.0 & 0.2 & 0.14 & 0.42 \\
& June 08a & 1119 & 1.0 & 0.4 & 1.5 & 0.65 & 0.73 \\
& June 08b & - & - & - & - & - & - \\
& June 10a & 1066 & 0.2 & 0.1 & 0.2 & 0.38 & 0.57 \\
& June 12a & 1048 & 0.2 & 0.0 & 0.2 & 0.64 & 0.80 \\
& June 12b & 952 & 0.2 & 0.0 & 0.1 & 0.28 & 0.53 \\
& June 14a & 1117 & 1.0 & 0.4 & 2.0 & 0.17 & 0.40 \\
& June 15a & 1054 & 1.0 & 1.8 & 2.7 & 0.80 & 0.58 \\
& June 15b & 1067 & 0.2 & 0.1 & 0.2 & 0.54 & 0.69 \\
& June 18a & 1097 & 0.2 & 0.0 & 0.3 & 0.49 & 0.65 \\
& June 19a & 995 & 0.2 & 0.0 & 0.1 & 0.70 & 0.81 \\
& June 21a & 1080 & 0.8 & 0.5 & 1.4 & 0.32 & 0.53 \\
& June 21b & 1045 & 0.2 & 0.1 & 0.3 & 0.20 & 0.40 \\
& June 23a & 1129 & 0.4 & 0.1 & 0.9 & 0.67 & 0.79 \\
& June 23b & 975 & 0.2 & 0.0 & 0.2 & 0.49 & 0.66 \\
& June 24a & 1083 & 1.1 & 0.2 & 1.9 & 0.32 & 0.55 \\
& June 24b & 899 & 0.4 & 0.6 & 1.2 & 0.74 & 0.49 \\
& June 27a & 1131 & 0.1 & 0.2 & 0.5 & 0.64 & 0.52 \\
& June 28a & 1054 & 0.5 & 1.1 & 2.6 & 0.29 & 0.28 \\
& June 28b & 749 & 0.2 & 0.0 & 0.1 & 0.25 & 0.50 \\
\hline
\end{tabular}


Table S20. Performance of simulated nitrogen dioxide $\left(\mathrm{NO}_{2}\right)$ from the DEF_ANT simulation in terms of bias, RMSE, R, and IA for each for the G-1 flights.

\begin{tabular}{cccccccc}
\hline \multirow{2}{*}{ Variable } & $\begin{array}{c}\text { Flight } \\
\text { Date }\end{array}$ & $\begin{array}{c}\text { Number of } \\
\text { Data Points }\end{array}$ & $\begin{array}{c}\text { Observed } \\
\text { Mean }\end{array}$ & Bias & RMSE & R & IA \\
\hline \multirow{6}{*}{ June 03a } & 777 & 0.7 & 0.2 & 0.8 & 0.66 & 0.72 \\
& June 06a & 1016 & 0.6 & 0.7 & 1.3 & 0.84 & 0.61 \\
& June 06b & 899 & 0.4 & 0.1 & 0.4 & 0.45 & 0.59 \\
& June 08a & 1061 & 2.2 & -0.4 & 1.6 & 0.67 & 0.79 \\
& June 08b & - & - & - & - & - & - \\
& June 10a & 965 & 0.6 & 0.1 & 0.3 & 0.42 & 0.60 \\
& June 12a & 995 & 0.4 & 0.0 & 0.3 & 0.73 & 0.84 \\
& June 12b & 900 & 0.5 & 0.1 & 0.3 & 0.53 & 0.64 \\
& June 14a & 1060 & 2.2 & 1.2 & 3.4 & 0.33 & 0.51 \\
& June 15a & 1003 & 1.7 & 2.3 & 3.0 & 0.81 & 0.60 \\
& June 15b & 1009 & 0.9 & 0.5 & 1.0 & 0.46 & 0.60 \\
& June 18a & 1042 & 0.9 & 0.0 & 0.9 & 0.66 & 0.76 \\
& June 19a & 948 & 0.0 & 0.9 & 1.1 & -0.27 & 0.03 \\
& June 21a & 1024 & 1.8 & 0.5 & 1.8 & 0.46 & 0.65 \\
& June 21b & 991 & 0.7 & 0.5 & 1.3 & 0.34 & 0.36 \\
& June 23a & 1072 & 0.9 & 0.3 & 1.6 & 0.73 & 0.80 \\
& June 23b & 928 & 0.9 & 0.4 & 0.9 & 0.61 & 0.66 \\
& June 24a & 1027 & 1.7 & 0.0 & 2.0 & 0.41 & 0.65 \\
& June 24b & 856 & 1.1 & 1.3 & 2.5 & 0.74 & 0.56 \\
& June 27a & 1078 & 0.5 & 0.7 & 1.4 & 0.62 & 0.46 \\
& June 28a & 1001 & 2.0 & 3.2 & 6.4 & 0.50 & 0.48 \\
& June 28b & 709 & 1.0 & 0.2 & 0.6 & 0.40 & 0.62 \\
\hline
\end{tabular}


Table S21. Performance of simulated sulfur dioxide $\left(\mathrm{SO}_{2}\right)$ from the DEF_ANT simulation in terms of bias, RMSE, R, and IA for each for the G-1 flights.

\begin{tabular}{cccccccc}
\hline \multirow{2}{*}{ Variable } & $\begin{array}{c}\text { Flight } \\
\text { Date }\end{array}$ & $\begin{array}{c}\text { Number of } \\
\text { Data Points }\end{array}$ & $\begin{array}{c}\text { Observed } \\
\text { Mean }\end{array}$ & Bias & RMSE & R & IA \\
\hline \multirow{6}{*}{ June 03a } & 808 & -0.3 & 0.5 & 1.0 & -0.08 & 0.34 \\
& June 06a & 1048 & 0.2 & -0.1 & 0.8 & 0.11 & 0.22 \\
& June 06b & 941 & -0.1 & 0.2 & 0.8 & 0.00 & 0.23 \\
& June 08a & - & - & - & - & - & - \\
& June 08b & - & - & - & - & - & - \\
& June 10a & - & - & - & - & - & - \\
$\mathrm{SO}_{2}$ & June 12a & - & - & - & - & - & - \\
& June 12b & - & - & - & - & - & - \\
& June 14a & - & - & - & - & - & - \\
& June 15a & 1037 & 0.8 & 0.0 & 0.4 & 0.69 & 0.82 \\
& June 15b & 1046 & 0.8 & -0.4 & 0.7 & 0.31 & 0.53 \\
& June 18a & 1082 & 0.8 & -0.7 & 1.1 & 0.13 & 0.43 \\
& June 19a & 1028 & 0.7 & -0.3 & 0.5 & 0.51 & 0.63 \\
& June 21a & 1071 & 0.6 & -0.3 & 0.8 & 0.11 & 0.43 \\
& June 21b & 1037 & 0.5 & -0.2 & 0.6 & 0.40 & 0.60 \\
& June 23a & 1112 & 0.7 & -0.4 & 0.6 & 0.41 & 0.53 \\
& June 23b & 955 & 1.0 & -0.7 & 0.9 & 0.39 & 0.46 \\
& June 24a & 944 & 0.5 & 0.1 & 0.9 & 0.19 & 0.44 \\
& June 24b & 889 & 1.0 & -0.7 & 0.9 & 0.30 & 0.49 \\
June 27a & 1093 & 0.6 & -0.1 & 0.6 & 0.50 & 0.55 \\
& June 28a & 1031 & 0.9 & -0.6 & 1.0 & 0.10 & 0.45 \\
& June 28b & 722 & 0.6 & -0.5 & 0.8 & 0.29 & 0.44 \\
\hline
\end{tabular}


Table S22. Performance of simulated ozone $\left(\mathrm{O}_{3}\right)$ from the DEF_ANT simulation in terms of bias, RMSE, R, and IA for each for the G-1 flights.

\begin{tabular}{cccccccc}
\hline \multirow{2}{*}{ Variable } & $\begin{array}{c}\text { Flight } \\
\text { Date }\end{array}$ & $\begin{array}{c}\text { Number of } \\
\text { Data Points }\end{array}$ & $\begin{array}{c}\text { Observed } \\
\text { Mean }\end{array}$ & Bias & RMSE & R & IA \\
\hline \multirow{6}{*}{ June 03a } & 835 & 38.2 & -9.2 & 10.7 & 0.55 & 0.54 \\
& June 06a & 1132 & 29.0 & -1.8 & 6.8 & 0.48 & 0.70 \\
& June 06b & 969 & 48.9 & -10.3 & 17.9 & -0.07 & 0.32 \\
& June 08a & 1152 & 44.0 & -5.0 & 10.8 & 0.83 & 0.85 \\
& June 08b & 1194 & 60.2 & -3.2 & 7.3 & 0.79 & 0.86 \\
& June 10a & 1088 & 43.5 & -4.7 & 5.7 & 0.51 & 0.51 \\
& June 12a & 1078 & 37.2 & 5.3 & 5.8 & 0.80 & 0.57 \\
& June 12b & - & - & - & - & - & - \\
& June 14a & 1075 & 44.9 & 6.8 & 9.8 & 0.49 & 0.58 \\
& June 15a & 1065 & 36.4 & -1.2 & 5.8 & 0.81 & 0.89 \\
& June 15b & 1082 & 63.5 & 6.1 & 9.6 & 0.09 & 0.36 \\
& June 18a & 1145 & 52.1 & 1.0 & 8.6 & 0.64 & 0.80 \\
& June 19a & 1101 & 49.1 & 7.7 & 10.3 & 0.28 & 0.36 \\
& June 21a & 1128 & 34.8 & 0.8 & 5.5 & 0.61 & 0.77 \\
& June 21b & 1035 & 55.9 & 4.7 & 9.2 & 0.02 & 0.40 \\
& June 23a & 1167 & 58.5 & 7.2 & 10.9 & 0.81 & 0.83 \\
& June 23b & 1024 & 71.5 & 18.4 & 20.8 & 0.39 & 0.35 \\
& June 24a & 1125 & 32.2 & 6.0 & 14.2 & 0.59 & 0.66 \\
& June 24b & 951 & 44.5 & 1.1 & 7.2 & 0.52 & 0.69 \\
& June 27a & 1152 & 48.5 & 18.0 & 21.2 & 0.26 & 0.29 \\
& June 28a & 1121 & 55.4 & 11.4 & 21.5 & 0.34 & 0.56 \\
& June 28b & 759 & 65.6 & 10.4 & 17.7 & 0.53 & 0.66 \\
\hline
\end{tabular}


Table S23. Performance of simulated isoprene from the DEF_ANT simulation in terms of bias, RMSE, R, and IA for each for the G-1 flights.

\begin{tabular}{cccccccc}
\hline \multirow{2}{*}{ Variable } & $\begin{array}{c}\text { Flight } \\
\text { Date }\end{array}$ & $\begin{array}{c}\text { Number of } \\
\text { Data Points }\end{array}$ & $\begin{array}{c}\text { Observed } \\
\text { Mean }\end{array}$ & Bias & RMSE & R & IA \\
\hline \multirow{5}{*}{ June 03a } & 854 & -0.2 & 0.2 & 0.3 & 0.71 & 0.44 \\
& June 06a & 1133 & 0.4 & -0.3 & 0.6 & 0.61 & 0.52 \\
& June 06b & 705 & 0.1 & -0.1 & 0.2 & 0.25 & 0.46 \\
& June 08a & 1190 & 0.4 & -0.3 & 0.5 & 0.61 & 0.47 \\
& June 08b & 1140 & 0.0 & 0.0 & 0.4 & 0.34 & 0.19 \\
& June 10a & 1113 & 0.1 & 0.0 & 0.1 & 0.47 & 0.61 \\
& June 12a & 1114 & 0.2 & -0.1 & 0.2 & 0.69 & 0.81 \\
& June 12b & - & - & - & - & - & - \\
& June 14a & 1168 & 0.3 & -0.1 & 0.4 & 0.25 & 0.43 \\
& June 15a & 1110 & 0.3 & -0.2 & 0.3 & 0.41 & 0.55 \\
& June 15b & 1115 & 0.5 & -0.4 & 0.6 & 0.47 & 0.51 \\
& June 18a & 632 & 0.2 & -0.1 & 0.6 & 0.17 & 0.13 \\
& June 19a & 1071 & 1.0 & -0.8 & 0.9 & 0.28 & 0.28 \\
& June 21a & 1075 & 0.4 & -0.3 & 0.3 & 0.53 & 0.52 \\
& June 21b & 1126 & 0.4 & -0.3 & 0.4 & 0.57 & 0.47 \\
& June 23a & 1079 & 0.6 & -0.4 & 0.7 & 0.73 & 0.64 \\
& June 23b & 1106 & 1.2 & -1.1 & 1.5 & 0.32 & 0.47 \\
& June 24a & 1173 & 0.3 & -0.3 & 0.5 & -0.09 & 0.33 \\
June 24b & 1034 & 0.8 & -0.7 & 1.1 & -0.07 & 0.45 \\
& June 27a & 840 & 1.6 & -1.2 & 2.3 & 0.83 & 0.54 \\
& June 28a & 1105 & 1.5 & -1.2 & 2.3 & 0.76 & 0.51 \\
& June 28b & 755 & 1.1 & -0.9 & 1.4 & 0.61 & 0.48 \\
\hline
\end{tabular}


Table S24. Performance of simulated methyl-vinyl-ketone + methacrolein (MVK+MACR) from the DEF_ANT simulation in terms of bias, RMSE, R, and IA for each for the G-1 flights.

\begin{tabular}{cccccccc}
\hline \multirow{2}{*}{ Variable } & $\begin{array}{c}\text { Flight } \\
\text { Date }\end{array}$ & $\begin{array}{c}\text { Number of } \\
\text { Data Points }\end{array}$ & $\begin{array}{c}\text { Observed } \\
\text { Mean }\end{array}$ & Bias & RMSE & R & IA \\
\hline \multirow{6}{*}{ June 03a } & 854 & 0.0 & 0.0 & 0.1 & 0.68 & 0.59 \\
& June 06a & 1133 & 0.4 & -0.2 & 0.3 & 0.72 & 0.67 \\
& June 06b & 705 & 0.2 & 0.0 & 0.1 & 0.65 & 0.76 \\
& June 08a & 1190 & 0.4 & -0.2 & 0.4 & 0.62 & 0.56 \\
& June 08b & 1140 & 0.7 & -0.6 & 0.7 & 0.52 & 0.50 \\
& June 10a & 1113 & 0.1 & -0.1 & 0.1 & 0.72 & 0.73 \\
MVK+ + ppbv) & June 12a & 1114 & 0.2 & 0.0 & 0.1 & 0.60 & 0.75 \\
& June 12b & - & - & - & - & - & - \\
& June 14a & 1168 & 0.4 & -0.1 & 0.3 & 0.39 & 0.54 \\
& June 15a & 1110 & 0.4 & -0.1 & 0.3 & 0.78 & 0.71 \\
& June 15b & 1115 & 0.6 & -0.4 & 0.6 & 0.63 & 0.53 \\
& June 19a & 1033 & 0.1 & 0.0 & 0.1 & 0.48 & 0.52 \\
& June 21a & 1075 & 0.3 & -0.1 & 0.2 & 0.48 & 0.62 \\
& June 21b & 1126 & 0.3 & -0.1 & 0.2 & 0.47 & 0.55 \\
& June 23a & 1079 & 0.8 & -0.4 & 0.6 & 0.76 & 0.70 \\
& June 23b & 1106 & 0.9 & -0.8 & 1.1 & 0.63 & 0.49 \\
& June 24a & 1173 & 0.3 & -0.2 & 0.4 & 0.28 & 0.42 \\
& June 24b & 1034 & 1.0 & -0.9 & 1.3 & 0.11 & 0.47 \\
& June 27a & 840 & 1.4 & -0.9 & 1.6 & 0.84 & 0.60 \\
& June 28a & 1106 & 2.3 & -1.7 & 2.4 & 0.64 & 0.51 \\
& June 28b & 755 & 0.9 & -0.5 & 0.8 & 0.17 & 0.45 \\
\hline
\end{tabular}


Table S25. Performance of simulated toluene from the DEF_ANT simulation in terms of bias, RMSE, R, and IA for each for the G-1 flights.

\begin{tabular}{cccccccc}
\hline \multirow{2}{*}{ Variable } & $\begin{array}{c}\text { Flight } \\
\text { Date }\end{array}$ & $\begin{array}{c}\text { Number of } \\
\text { Data Points }\end{array}$ & $\begin{array}{c}\text { Observed } \\
\text { Mean }\end{array}$ & Bias & RMSE & R & IA \\
\hline \multirow{5}{*}{ June 03a } & 851 & 0.1 & -0.1 & 0.2 & 0.32 & 0.51 \\
& June 06a & - & - & - & - & - & - \\
& June 06b & 705 & 0.1 & 0.0 & 0.0 & 0.26 & 0.54 \\
& June 08a & 1190 & 0.1 & 0.0 & 0.1 & 0.40 & 0.52 \\
& June 08b & 1140 & 0.0 & 0.1 & 0.1 & 0.45 & 0.45 \\
& June 10a & 1113 & 0.0 & 0.0 & 0.0 & 0.03 & 0.39 \\
& June 12a & 1114 & 0.0 & 0.0 & 0.0 & 0.23 & 0.51 \\
& June 12b & - & - & - & - & - & - \\
& June 14a & 1168 & 0.1 & 0.1 & 0.2 & 0.25 & 0.48 \\
& June 15a & 1110 & 0.1 & 0.2 & 0.2 & 0.43 & 0.31 \\
& June 15b & 1115 & 0.1 & 0.0 & 0.1 & 0.23 & 0.49 \\
& June 18a & 633 & 0.1 & 0.0 & 0.1 & 0.21 & 0.53 \\
& June 19a & 1071 & 0.1 & 0.0 & 0.0 & 0.40 & 0.64 \\
& June 21a & 1075 & 0.1 & 0.1 & 0.1 & 0.43 & 0.56 \\
& June 21b & 1126 & 0.1 & 0.0 & 0.1 & 0.11 & 0.40 \\
& June 23a & 1079 & 0.1 & 0.0 & 0.2 & 0.45 & 0.49 \\
& June 23b & 1106 & 0.1 & 0.0 & 0.1 & -0.01 & 0.38 \\
& June 24a & 1173 & 0.1 & 0.0 & 0.1 & 0.21 & 0.40 \\
& June 24b & 1034 & 0.1 & 0.1 & 0.2 & 0.33 & 0.45 \\
& June 27a & 840 & 0.1 & 0.0 & 0.1 & 0.58 & 0.63 \\
& June 28a & 1106 & 0.2 & 0.2 & 0.3 & 0.49 & 0.49 \\
& June 28b & 755 & 0.1 & -0.1 & 0.1 & 0.24 & 0.46 \\
\hline
\end{tabular}


Table S26. Performance of simulated terpene (TERP) from the DEF_ANT simulation in terms of bias, RMSE, R, and IA for each for the G-1 flights.

\begin{tabular}{cccccccc}
\hline \multirow{2}{*}{ Variable } & Flight & $\begin{array}{c}\text { Number } \\
\text { of Data } \\
\text { Points }\end{array}$ & $\begin{array}{c}\text { Observed } \\
\text { Mean }\end{array}$ & Bias & RMSE & R & IA \\
\hline \multirow{6}{*}{ Dane 03a } & 854 & 0.0 & 0.0 & 0.1 & 0.43 & 0.43 \\
& June 06a & 1133 & 0.0 & 0.0 & 0.1 & 0.02 & 0.37 \\
& June 06b & 705 & 0.0 & 0.0 & 0.1 & 0.02 & 0.27 \\
& June 08a & 1190 & 0.0 & 0.0 & 0.1 & 0.05 & 0.44 \\
& June 08b & 1140 & 0.0 & 0.0 & 0.1 & 0.07 & 0.40 \\
& June 10a & 1113 & 0.0 & 0.0 & 0.0 & 0.01 & 0.39 \\
& June 12a & 1114 & 0.0 & 0.0 & 0.0 & 0.10 & 0.43 \\
& June 12b & - & - & - & - & - & - \\
& June 14a & 1168 & 0.1 & -0.1 & 0.1 & -0.03 & 0.41 \\
& June 15a & 1110 & 0.0 & 0.0 & 0.1 & -0.09 & 0.39 \\
& June 15b & 1115 & 0.1 & -0.1 & 0.1 & 0.28 & 0.46 \\
& June 18a & 633 & 0.1 & 0.0 & 0.1 & 0.16 & 0.45 \\
& June 19a & 1071 & 0.1 & -0.1 & 0.1 & -0.08 & 0.42 \\
& June 21a & 1075 & 0.1 & 0.0 & 0.1 & 0.14 & 0.45 \\
& June 21b & 1126 & 0.1 & -0.1 & 0.1 & 0.15 & 0.44 \\
& June 23a & 1079 & 0.1 & -0.1 & 0.1 & 0.35 & 0.50 \\
& June 23b & 1106 & 0.1 & -0.1 & 0.1 & 0.23 & 0.43 \\
& June 24a & 1173 & 0.1 & -0.1 & 0.1 & 0.07 & 0.44 \\
& June 24b & 1034 & 0.1 & -0.1 & 0.1 & -0.16 & 0.41 \\
& June 27a & 840 & 0.1 & -0.1 & 0.1 & 0.46 & 0.51 \\
& June 28a & 1106 & 0.1 & -0.1 & 0.1 & 0.25 & 0.46 \\
June 28b & 755 & 0.1 & -0.1 & 0.1 & 0.16 & 0.44 \\
\hline
\end{tabular}


Table S27. Performance of simulated carbon monoxide (CO) from the DEF_ANT simulation in terms of bias, RMSE, R, and IA for each for the WP-3D flights.

\begin{tabular}{cccccccc}
\hline \multirow{2}{*}{ Variable } & Flight Date & $\begin{array}{c}\text { Number } \\
\text { of Data } \\
\text { Points }\end{array}$ & $\begin{array}{c}\text { Observed } \\
\text { Mean }\end{array}$ & Bias & RMSE & R & IA \\
\hline & May 4 & 16011 & 166.3 & 42.5 & 69.4 & 0.75 & 0.79 \\
& May 7 & 23212 & 161.7 & 9.3 & 35.5 & 0.78 & 0.86 \\
& May 8 & 24182 & 177.0 & 8.6 & 44.2 & 0.80 & 0.89 \\
& May 11 & 24549 & 145.6 & 0.0 & 13.6 & 0.82 & 0.90 \\
CO & May 12 & 26169 & 153.7 & 10.4 & 26.4 & 0.85 & 0.90 \\
& May 14 & 20857 & 172.1 & 29.6 & 56.4 & 0.83 & 0.87 \\
& May 16 & 26495 & 168.6 & 30.0 & 50.9 & 0.90 & 0.89 \\
& May 19 & 22960 & 173.5 & 15.9 & 52.3 & 0.86 & 0.92 \\
& May 212 & 10366 & 150.8 & -5.7 & 16.6 & 0.90 & 0.94 \\
& May 22 & 21538 & 142.3 & 0.2 & 20.3 & 0.80 & 0.84 \\
& May 23 & 19728 & 150.9 & 27.5 & 59.4 & 0.57 & 0.58 \\
& May 24 & 20125 & 164.2 & 49.9 & 80.8 & 0.76 & 0.69 \\
& May 25 & 21126 & 158.6 & 12.9 & 34.4 & 0.73 & 0.83 \\
& June 3 & 22894 & 179.3 & 27.0 & 61.2 & 0.80 & 0.86 \\
& June 14 & 25077 & 123.3 & 11.0 & 30.2 & 0.82 & 0.89 \\
& June 16 & 23692 & 133.2 & 8.1 & 35.4 & 0.82 & 0.80 \\
& June 18 & 24262 & 120.2 & 6.9 & 20.3 & 0.88 & 0.92 \\
& June 20 & 24350 & 164.8 & 21.0 & 54.2 & 0.79 & 0.79 \\
& June 22 & 4536 & 136.3 & 25.9 & 50.9 & 0.62 & 0.68 \\
\hline
\end{tabular}


Table S28. Performance of simulated nitrogen oxide (NO) from the DEF_ANT simulation in terms of bias, RMSE, R, and IA for each for the WP-3D flights.

\begin{tabular}{cccccccc}
\hline \multirow{2}{*}{ Variable } & $\begin{array}{c}\text { Flight } \\
\text { Date }\end{array}$ & $\begin{array}{c}\text { Number } \\
\text { of Data } \\
\text { Points }\end{array}$ & $\begin{array}{c}\text { Observed } \\
\text { Mean }\end{array}$ & Bias & RMSE & R & IA \\
\hline \multirow{5}{*}{ May 4 } & 15868 & 1.0 & 0.6 & 2.4 & 0.63 & 0.74 \\
& May 7 & 23049 & 0.4 & 0.4 & 1.5 & 0.59 & 0.72 \\
& May 8 & 23923 & 0.6 & 0.2 & 2.7 & 0.19 & 0.32 \\
& May 11 & 21719 & 0.4 & 0.1 & 0.7 & 0.87 & 0.91 \\
& May 12 & 25402 & 0.6 & 0.1 & 1.5 & 0.65 & 0.78 \\
& May 14 & - & - & - & - & - & - \\
& May 16 & 26574 & 0.2 & 0.5 & 1.5 & 0.72 & 0.56 \\
& May 19 & 22654 & 2.3 & 1.0 & 3.5 & 0.77 & 0.83 \\
& May 21 & 8785 & 0.8 & -0.3 & 3.0 & 0.49 & 0.54 \\
& May 22 & 20434 & 0.1 & 0.2 & 1.3 & 0.60 & 0.54 \\
& May 23 & 19471 & 0.0 & 0.0 & 0.9 & 0.46 & 0.14 \\
& May 24 & 19906 & 0.0 & 0.1 & 1.2 & 0.28 & 0.28 \\
& May 25 & 21034 & 0.1 & 0.2 & 2.1 & 0.17 & 0.07 \\
& June 3 & 22893 & 0.2 & 0.3 & 1.8 & 0.62 & 0.71 \\
& June 14 & 23681 & 0.2 & 0.1 & 1.4 & 0.80 & 0.84 \\
& June 16 & 23026 & 0.4 & 0.3 & 1.7 & 0.87 & 0.76 \\
& June 18 & 23687 & 0.3 & 0.2 & 1.0 & 0.77 & 0.74 \\
& June 20 & 23911 & 0.4 & 1.7 & 5.1 & 0.37 & 0.19 \\
& June 22 & 4357 & 0.4 & 0.1 & 1.6 & 0.50 & 0.67 \\
\hline
\end{tabular}


Table S29. Performance of simulated nitrogen dioxide $\left(\mathrm{NO}_{2}\right)$ from the DEF_ANT simulation in terms of bias, RMSE, R, and IA for each for the WP-3D flights.

\begin{tabular}{cccccccc}
\hline \multirow{2}{*}{ Variable } & $\begin{array}{c}\text { Flight } \\
\text { Date }\end{array}$ & $\begin{array}{c}\text { Number } \\
\text { of Data } \\
\text { Points }\end{array}$ & $\begin{array}{c}\text { Observed } \\
\text { Mean }\end{array}$ & Bias & RMSE & R & IA \\
\hline \multirow{5}{*}{ May 4 } & 13716 & 3.0 & 1.1 & 4.7 & 0.73 & 0.84 \\
& May 7 & 19543 & 1.3 & 1.1 & 4.7 & 0.35 & 0.51 \\
& May 8 & 23562 & 2.0 & 0.4 & 3.3 & 0.63 & 0.77 \\
& May 11 & 20335 & 0.9 & 0.1 & 0.9 & 0.91 & 0.95 \\
& May 12 & 24752 & 1.6 & 0.2 & 2.4 & 0.82 & 0.88 \\
& May 14 & - & - & - & - & - & - \\
& May 16 & 25836 & 0.9 & 1.5 & 3.9 & 0.87 & 0.69 \\
& May 19 & 22016 & 4.1 & 1.2 & 4.1 & 0.85 & 0.90 \\
& May 212 & 8569 & 1.1 & -0.1 & 2.4 & 0.69 & 0.78 \\
& May 22 & 19838 & 0.9 & 0.7 & 1.9 & 0.85 & 0.82 \\
& May 23 & 19019 & 1.4 & 2.6 & 5.7 & 0.61 & 0.56 \\
& May 24 & 19676 & 1.5 & 3.5 & 7.1 & 0.61 & 0.53 \\
& May 25 & 20812 & 1.5 & 0.9 & 4.1 & 0.54 & 0.58 \\
& June 3 & 22697 & 2.3 & 4.1 & 8.3 & 0.52 & 0.55 \\
& June 14 & 22767 & 0.8 & 0.4 & 2.1 & 0.83 & 0.89 \\
& June 16 & 22390 & 1.0 & 0.6 & 2.3 & 0.91 & 0.82 \\
& June 18 & 23234 & 0.9 & 0.4 & 1.5 & 0.84 & 0.84 \\
& June 20 & 23475 & 1.1 & 2.6 & 6.0 & 0.61 & 0.44 \\
& June 22 & 4228 & 0.9 & 0.3 & 2.5 & 0.52 & 0.69 \\
\hline
\end{tabular}


Table S30. Performance of simulated ammonia $\left(\mathrm{NH}_{3}\right)$ from the DEF_ANT simulation in terms of bias, RMSE, R, and IA for each for the WP-3D flights.

\begin{tabular}{cccccccc}
\hline \multirow{2}{*}{ Variable } & $\begin{array}{c}\text { Flight } \\
\text { Date }\end{array}$ & $\begin{array}{c}\text { Number } \\
\text { of Data } \\
\text { Points }\end{array}$ & $\begin{array}{c}\text { Observed } \\
\text { Mean }\end{array}$ & Bias & RMSE & R & IA \\
\hline \multirow{5}{*}{ May 4 } & 8506 & 3.7 & -1.6 & 8.9 & 0.44 & 0.28 \\
& May 7 & 14716 & 24.2 & -19.5 & 42.9 & 0.56 & 0.36 \\
& May 8 & 13058 & 5.0 & -2.9 & 7.7 & 0.59 & 0.41 \\
& May 11 & 10948 & 4.3 & -3.3 & 4.7 & 0.49 & 0.51 \\
& May 12 & 16250 & 11.2 & -8.7 & 17.9 & 0.70 & 0.42 \\
& May 14 & 14387 & 5.3 & -3.5 & 12.2 & 0.57 & 0.28 \\
& May 16 & 20454 & 3.2 & -1.8 & 13.1 & 0.42 & 0.16 \\
& May 19 & 18686 & 13.3 & -10.9 & 49.3 & 0.54 & 0.15 \\
& May 21 & 8783 & 1.7 & -0.9 & 5.0 & 0.47 & 0.50 \\
& May 22 & 17075 & 5.8 & -3.3 & 15.6 & 0.17 & 0.18 \\
& May 23 & 18552 & 0.9 & 0.5 & 2.1 & 0.46 & 0.55 \\
& May 24 & 18966 & 3.0 & -0.9 & 9.2 & 0.18 & 0.20 \\
& May 25 & 19875 & 3.3 & -1.4 & 11.3 & 0.46 & 0.33 \\
& June 3 & 17383 & 3.2 & -0.9 & 6.9 & 0.03 & 0.20 \\
& June 14 & 19974 & 4.1 & -2.8 & 5.7 & 0.75 & 0.57 \\
& June 16 & 21467 & 7.8 & -4.2 & 10.4 & 0.65 & 0.50 \\
& June 18 & 19080 & 7.7 & -5.9 & 18.0 & 0.35 & 0.25 \\
& June 20 & 22002 & 2.4 & -0.2 & 4.0 & 0.47 & 0.57 \\
& June 22 & 3573 & 5.7 & -3.9 & 8.0 & 0.68 & 0.47 \\
\hline
\end{tabular}


Table S31. Performance of simulated sulfur dioxide $\left(\mathrm{SO}_{2}\right)$ from the DEF_ANT simulation in terms of bias, RMSE, R, and IA for each for the WP-3D flights.

\begin{tabular}{|c|c|c|c|c|c|c|c|}
\hline \multirow[t]{9}{*}{ Variable } & $\begin{array}{l}\text { Flight } \\
\text { Date }\end{array}$ & $\begin{array}{c}\text { Number } \\
\text { of Data } \\
\text { Points }\end{array}$ & $\begin{array}{c}\text { Observed } \\
\text { Mean }\end{array}$ & Bias & RMSE & $\mathbf{R}$ & IA \\
\hline & May 4 & 15413 & 0.4 & -0.1 & 0.8 & 0.41 & 0.60 \\
\hline & May 7 & 22412 & 0.8 & -0.5 & 0.9 & 0.38 & 0.54 \\
\hline & May 8 & 23025 & 0.7 & -0.3 & 0.9 & 0.45 & 0.62 \\
\hline & May 11 & 23401 & 0.1 & 0.0 & 0.3 & 0.44 & 0.61 \\
\hline & May 12 & 24820 & 0.4 & -0.3 & 0.7 & 0.31 & 0.49 \\
\hline & May 14 & 20161 & 0.5 & 0.0 & 0.6 & 0.80 & 0.89 \\
\hline & May 16 & 25416 & 0.3 & 0.1 & 0.7 & 0.40 & 0.53 \\
\hline & May 19 & 22073 & 0.3 & 0.0 & 0.6 & 0.58 & 0.74 \\
\hline \multirow{11}{*}{$\begin{array}{c}\mathrm{SO}_{2} \\
(\mathrm{ppbV})\end{array}$} & May 212 & 9942 & 0.3 & -0.3 & 1.2 & 0.15 & 0.16 \\
\hline & May 22 & 20576 & 0.4 & -0.3 & 0.6 & 0.26 & 0.50 \\
\hline & May 23 & 19016 & 0.4 & -0.1 & 1.4 & 0.24 & 0.23 \\
\hline & May 24 & 19306 & 0.6 & -0.1 & 1.1 & 0.44 & 0.52 \\
\hline & May 25 & 20316 & 0.4 & -0.1 & 1.1 & 0.16 & 0.24 \\
\hline & June 3 & 22020 & 0.6 & -0.1 & 0.7 & 0.47 & 0.62 \\
\hline & June 14 & 23709 & 0.4 & -0.2 & 0.6 & 0.49 & 0.53 \\
\hline & June 16 & 22677 & 0.7 & -0.5 & 0.9 & 0.22 & 0.47 \\
\hline & June 18 & 23319 & 0.4 & -0.2 & 0.7 & 0.27 & 0.49 \\
\hline & June 20 & 23384 & 0.4 & 0.3 & 1.6 & 0.26 & 0.29 \\
\hline & June 22 & 4307 & 0.5 & -0.2 & 0.9 & 0.11 & 0.35 \\
\hline
\end{tabular}


Table S32. Performance of simulated ozone $\left(\mathrm{O}_{3}\right)$ from the DEF_ANT simulation in terms of bias, RMSE, R, and IA for each for the WP-3D flights.

\begin{tabular}{cccccccc}
\hline Variable & Flight Date & $\begin{array}{c}\text { Number } \\
\text { of Data } \\
\text { Points }\end{array}$ & $\begin{array}{c}\text { Observed } \\
\text { Mean }\end{array}$ & Bias & RMSE & R & IA \\
\hline & May 4 & 16552 & 68.0 & -2.3 & 8.5 & 0.54 & 0.71 \\
& May 7 & 23787 & 62.6 & -5.4 & 10.0 & 0.60 & 0.70 \\
& May 8 & 24401 & 75.7 & -8.9 & 12.4 & 0.75 & 0.75 \\
& May 11 & 25079 & 50.9 & -4.1 & 15.9 & 0.69 & 0.69 \\
& May 12 & 26879 & 51.7 & 4.0 & 10.6 & 0.52 & 0.64 \\
$\mathrm{O}_{3}$ & May 14 & - & - & - & - & - & - \\
& May 16 & 27326 & 66.6 & -1.5 & 13.1 & 0.63 & 0.78 \\
& May 19 & 23389 & 51.3 & -4.8 & 11.1 & 0.58 & 0.71 \\
& May 21 & 9855 & 57.5 & -10.4 & 13.4 & 0.87 & 0.74 \\
& May 22 & 21551 & 56.7 & -12.6 & 14.8 & 0.69 & 0.55 \\
& May 23 & 20158 & 63.9 & -10.8 & 13.5 & 0.61 & 0.59 \\
& May 24 & 20628 & 63.4 & -9.0 & 14.2 & 0.39 & 0.52 \\
& May 25 & 21733 & 49.9 & -2.2 & 13.5 & 0.43 & 0.65 \\
& June 3 & 23712 & 53.3 & -14.9 & 21.2 & 0.19 & 0.46 \\
& June 14 & 24837 & 58.8 & 1.7 & 9.7 & 0.74 & 0.86 \\
& June 16 & 24068 & 55.0 & -4.6 & 9.1 & 0.77 & 0.84 \\
& June 18 & 24639 & 51.2 & -4.8 & 10.2 & 0.79 & 0.85 \\
& June 20 & 24574 & 66.9 & -10.7 & 16.0 & 0.51 & 0.64 \\
& June 22 & 4598 & 68.7 & -5.1 & 12.1 & 0.44 & 0.59 \\
\hline
\end{tabular}


Table S33. Performance of simulated isoprene from the DEF_ANT simulation in terms of bias, RMSE, R, and IA for each for the WP-3D flights.

\begin{tabular}{cccccccc}
\hline \multirow{2}{*}{ Variable } & $\begin{array}{c}\text { Flight } \\
\text { Date }\end{array}$ & $\begin{array}{c}\text { Number } \\
\text { of Data } \\
\text { Points }\end{array}$ & $\begin{array}{c}\text { Observed } \\
\text { Mean }\end{array}$ & Bias & RMSE & R & IA \\
\hline & May 4 & 616 & 0.1 & 0.0 & 0.1 & 0.53 & 0.71 \\
& May 7 & 937 & 0.1 & 0.0 & 0.1 & 0.63 & 0.77 \\
& May 8 & 1371 & 0.1 & 0.0 & 0.1 & 0.56 & 0.73 \\
& May 11 & 1118 & 0.0 & 0.0 & 0.0 & 0.52 & 0.70 \\
& May 12 & 1464 & 0.0 & 0.0 & 0.1 & 0.68 & 0.81 \\
isoprene & May 14 & 1160 & 0.1 & 0.0 & 0.1 & 0.84 & 0.88 \\
& May 16 & 1448 & 0.0 & 0.0 & 0.1 & 0.75 & 0.81 \\
& May 19 & 912 & 0.1 & 0.0 & 0.1 & 0.76 & 0.85 \\
& May 21 & 465 & 0.0 & 0.0 & 0.0 & 0.69 & 0.78 \\
& May 22 & 1222 & 0.0 & 0.0 & 0.0 & 0.56 & 0.67 \\
& May 23 & 1122 & 0.0 & 0.0 & 0.1 & 0.36 & 0.56 \\
& May 24 & 1149 & 0.0 & 0.0 & 0.1 & 0.15 & 0.38 \\
& May 25 & 1199 & 0.0 & 0.0 & 0.0 & 0.18 & 0.37 \\
& June 3 & 1300 & 0.0 & 0.0 & 0.0 & 0.18 & 0.42 \\
& June 14 & 1326 & 0.1 & 0.0 & 0.2 & 0.66 & 0.78 \\
& June 16 & 1077 & 0.0 & 0.0 & 0.1 & 0.52 & 0.70 \\
& June 18 & 808 & 0.0 & 0.0 & 0.1 & 0.47 & 0.68 \\
& June 20 & 1419 & 0.1 & 0.0 & 0.1 & 0.59 & 0.74 \\
& June 22 & 267 & 0.1 & 0.0 & 0.2 & 0.35 & 0.52 \\
\hline & & & & & & &
\end{tabular}


Table S34. Performance of simulated methyl-vinyl-ketone + methacrolein (MVK+MACR) from the DEF_ANT simulation in terms of bias, RMSE, R, and IA for each for the WP-3D flights.

\begin{tabular}{cccccccc}
\hline \multirow{2}{*}{ Variable } & $\begin{array}{c}\text { Flight } \\
\text { Date }\end{array}$ & $\begin{array}{c}\text { Number } \\
\text { of Data } \\
\text { Points }\end{array}$ & $\begin{array}{c}\text { Observed } \\
\text { Mean }\end{array}$ & Bias & RMSE & R & IA \\
\hline \multirow{5}{*}{ May 4 } & 70 & 0.2 & 0.0 & 0.2 & 0.00 & 0.16 \\
& May 7 & 70 & 0.1 & 0.1 & 0.1 & 0.08 & 0.42 \\
& May 8 & 71 & 0.2 & 0.1 & 0.1 & 0.21 & 0.49 \\
& May 11 & 71 & 0.1 & 0.0 & 0.1 & -0.19 & 0.28 \\
& May 12 & 70 & 0.1 & 0.1 & 0.1 & -0.33 & 0.30 \\
MVK+ & May 14 & 70 & 0.1 & 0.0 & 0.1 & -0.48 & 0.12 \\
& May 16 & 71 & 0.1 & 0.1 & 0.1 & 0.08 & 0.37 \\
& May 19 & 71 & 0.1 & 0.1 & 0.1 & -0.02 & 0.39 \\
& May 212 & 71 & -0.1 & 0.2 & 0.5 & 0.23 & 0.28 \\
& May 22 & 71 & -0.3 & 0.4 & 0.7 & 0.55 & 0.39 \\
& May 23 & 58 & -0.1 & 0.3 & 0.6 & 0.21 & 0.38 \\
& May 24 & 71 & -0.1 & 0.2 & 0.5 & -0.12 & 0.27 \\
& May 25 & 65 & -0.1 & 0.2 & 0.5 & 0.09 & 0.30 \\
& June 3 & 71 & 0.0 & 0.1 & 0.5 & 0.07 & 0.20 \\
& June 14 & 71 & -0.1 & 0.3 & 0.8 & -0.23 & 0.33 \\
& June 16 & 70 & -0.2 & 0.4 & 0.7 & -0.25 & 0.37 \\
& June 18 & 71 & -0.1 & 0.4 & 0.7 & 0.08 & 0.36 \\
& June 20 & 71 & -0.2 & 0.5 & 0.9 & 0.10 & 0.41 \\
& June 22 & 71 & 0.2 & 0.0 & 0.3 & -0.16 & 0.12 \\
\hline & & & & & & &
\end{tabular}


Table S35. Performance of simulated terpene (TERP) from the DEF_ANT simulation in terms of bias, RMSE, R, and IA for each for the WP-3D flights.

\begin{tabular}{cccccccc}
\hline \multirow{2}{*}{ Variable } & $\begin{array}{c}\text { Flight } \\
\text { Date }\end{array}$ & $\begin{array}{c}\text { Number } \\
\text { of Data } \\
\text { Points }\end{array}$ & $\begin{array}{c}\text { Observed } \\
\text { Mean }\end{array}$ & Bias & RMSE & R & IA \\
\hline \multirow{5}{*}{ May 4 } & 745 & 0.0 & 0.0 & 0.0 & 0.51 & 0.46 \\
& May 7 & 1147 & 0.0 & 0.0 & 0.0 & 0.28 & 0.41 \\
& May 8 & 1202 & 0.0 & 0.0 & 0.0 & 0.50 & 0.49 \\
& May 11 & 1282 & 0.0 & 0.0 & 0.0 & 0.18 & 0.38 \\
& May 12 & 1464 & 0.0 & 0.0 & 0.0 & 0.46 & 0.41 \\
& May 14 & 1160 & 0.0 & 0.0 & 0.0 & 0.75 & 0.65 \\
& May 16 & 1448 & 0.0 & 0.0 & 0.0 & 0.54 & 0.59 \\
& May 19 & 1247 & 0.0 & 0.0 & 0.1 & 0.60 & 0.50 \\
& May 21 & 573 & 0.0 & 0.0 & 0.0 & 0.77 & 0.25 \\
& May 22 & 1221 & 0.0 & 0.0 & 0.0 & 0.24 & 0.21 \\
& May 23 & 1122 & 0.0 & 0.0 & 0.0 & 0.44 & 0.56 \\
& May 24 & 1148 & 0.0 & 0.0 & 0.0 & 0.42 & 0.49 \\
& May 25 & 1198 & 0.0 & 0.0 & 0.0 & 0.13 & 0.24 \\
& June 3 & 1300 & 0.0 & 0.0 & 0.0 & 0.18 & 0.40 \\
& June 14 & 1326 & 0.0 & 0.0 & 0.0 & 0.25 & 0.32 \\
& June 16 & 1388 & 0.0 & 0.0 & 0.0 & 0.49 & 0.32 \\
& June 18 & 1177 & 0.0 & 0.0 & 0.0 & 0.27 & 0.19 \\
& June 20 & 1302 & 0.0 & 0.0 & 0.0 & 0.42 & 0.59 \\
& June 22 & 266 & 0.0 & 0.0 & 0.1 & 0.65 & 0.28 \\
\hline & & & & & & &
\end{tabular}


Table S36. Performance of simulated formaldehyde $\left(\mathrm{CH}_{2} \mathrm{O}\right)$ from the DEF_ANT simulation in terms of bias, RMSE, R, and IA for each for the WP-3D flights.

\begin{tabular}{lccccccc}
\hline \multirow{2}{*}{ Variable } & $\begin{array}{c}\text { Flight } \\
\text { Date }\end{array}$ & $\begin{array}{c}\text { Number } \\
\text { of Data } \\
\text { Points }\end{array}$ & $\begin{array}{c}\text { Observed } \\
\text { Mean }\end{array}$ & Bias & RMSE & R & IA \\
\hline \multirow{3}{*}{ May 4 } & 780 & 1.9 & -0.4 & 0.9 & 0.81 & 0.86 \\
& May 7 & 1323 & 1.7 & -0.4 & 0.7 & 0.75 & 0.79 \\
& May 8 & 1372 & 2.1 & -1.0 & 1.3 & 0.83 & 0.73 \\
& May 11 & 1379 & 0.9 & -0.3 & 0.5 & 0.56 & 0.64 \\
& May 12 & 1464 & 1.4 & -0.4 & 0.6 & 0.76 & 0.79 \\
& May 14 & 1200 & 1.6 & -0.4 & 0.9 & 0.78 & 0.83 \\
$\mathrm{CH}_{2} \mathrm{O}$ & May 16 & 1501 & 1.6 & -0.5 & 0.9 & 0.88 & 0.86 \\
& May 19 & 1306 & 2.1 & -0.8 & 1.2 & 0.85 & 0.79 \\
& May 212 & 574 & 1.1 & -0.5 & 0.7 & 0.73 & 0.69 \\
& May 22 & 1222 & 1.1 & -0.3 & 0.5 & 0.50 & 0.58 \\
& May 23 & 1123 & 1.8 & -0.3 & 0.7 & 0.72 & 0.79 \\
& May 24 & 1150 & 2.1 & -0.3 & 0.8 & 0.73 & 0.79 \\
& May 25 & 1199 & 2.0 & -0.8 & 1.0 & 0.55 & 0.56 \\
& June 3 & 1300 & 2.6 & -1.0 & 1.2 & 0.80 & 0.66 \\
& June 14 & 1478 & 2.9 & -1.3 & 1.6 & 0.84 & 0.77 \\
& June 16 & 1388 & 2.1 & -0.8 & 1.0 & 0.72 & 0.66 \\
& June 18 & 1388 & 2.1 & -0.9 & 1.3 & 0.31 & 0.50 \\
& June 20 & 1419 & 2.7 & -1.3 & 1.5 & 0.81 & 0.66 \\
& June 22 & 267 & 2.7 & -1.5 & 1.6 & 0.50 & 0.44 \\
\hline & & & & & & &
\end{tabular}


Table S37. Performance of simulated black carbon (BC) from the DEF_ANT simulation in terms of bias, RMSE, R, and IA for each for the G-1, WP-3D, and CIRPAS Twin Otter flights.

\begin{tabular}{|c|c|c|c|c|c|c|}
\hline Flight Date & $\begin{array}{c}\text { Number } \\
\text { of Data } \\
\text { Points } \\
\end{array}$ & $\begin{array}{c}\text { Observed } \\
\text { Mean } \\
(\mu \mathrm{g} / \mathrm{m} 3) \\
\end{array}$ & $\begin{array}{c}\text { Bias } \\
(\mu \mathrm{g} / \mathrm{m} 3)\end{array}$ & RMSE & $\mathbf{R}$ & IA \\
\hline G-1, June $03 a$ & 787 & 0.03 & 0.03 & 0.04 & 0.36 & 0.40 \\
\hline G-1. June 06a & 1083 & 0.02 & 0.08 & 0.09 & 0.54 & 0.24 \\
\hline G-1, June $06 \mathrm{~b}$ & 658 & 0.02 & 0.07 & 0.09 & 0.10 & 0.14 \\
\hline G-1, June $08 \mathrm{a}$ & 1144 & 0.09 & 0.07 & 0.10 & 0.10 & 0.36 \\
\hline G-1, June $08 b$ & 883 & 0.07 & 0.08 & 0.09 & 0.67 & 0.41 \\
\hline G-1, June 10a & 786 & 0.03 & 0.03 & 0.04 & 0.32 & 0.29 \\
\hline G-1, June $12 \mathrm{a}$ & 1090 & 0.02 & 0.05 & 0.06 & 0.47 & 0.24 \\
\hline G-1, June $12 b$ & 713 & 0.02 & 0.05 & 0.05 & -0.02 & 0.16 \\
\hline G-1, June $14 \mathrm{a}$ & 1139 & 0.08 & 0.10 & 0.12 & 0.50 & 0.48 \\
\hline G-1, June $15 a$ & 1075 & 0.07 & 0.16 & 0.16 & 0.65 & 0.32 \\
\hline G-1, June $15 b$ & 830 & 0.09 & 0.08 & 0.09 & 0.58 & 0.46 \\
\hline G-1, June $18 \mathrm{a}$ & 786 & 0.05 & 0.04 & 0.07 & 0.17 & 0.33 \\
\hline G-1, June $19 a$ & - & - & - & - & - & - \\
\hline G-1, June $21 \mathrm{a}$ & 1107 & 0.07 & 0.12 & 0.18 & 0.21 & 0.31 \\
\hline G-1, June $21 \mathrm{~b}$ & 797 & 0.06 & 0.05 & 0.08 & 0.42 & 0.53 \\
\hline G-1, June $23 \mathrm{a}$ & 1145 & 0.09 & 0.09 & 0.10 & 0.44 & 0.39 \\
\hline G-1, June $23 b$ & 769 & 0.10 & 0.09 & 0.10 & 0.36 & 0.40 \\
\hline G-1, June $24 \mathrm{a}$ & 1140 & 0.06 & 0.07 & 0.10 & 0.06 & 0.36 \\
\hline G-1, June $24 b$ & 703 & 0.08 & 0.05 & 0.08 & 0.48 & 0.61 \\
\hline G-1, June $27 \mathrm{a}$ & 1185 & 0.07 & 0.15 & 0.16 & 0.73 & 0.27 \\
\hline G-1, June $28 \mathrm{a}$ & 1091 & 0.13 & 0.17 & 0.21 & 0.56 & 0.51 \\
\hline G-1, June $28 b$ & 579 & 0.11 & 0.06 & 0.09 & 0.11 & 0.42 \\
\hline WP-3D, May 4 & 8197 & 0.02 & 0.13 & 0.14 & 0.59 & 0.27 \\
\hline WP-3D, May 7 & 24831 & 0.08 & 0.07 & 0.12 & 0.73 & 0.74 \\
\hline WP-3D, May 8 & 25375 & 0.09 & 0.09 & 0.15 & 0.66 & 0.63 \\
\hline WP-3D, May 11 & 25780 & 0.03 & 0.05 & 0.08 & 0.65 & 0.64 \\
\hline WP-3D, May 12 & 25020 & 0.08 & 0.06 & 0.10 & 0.68 & 0.73 \\
\hline WP-3D, May 14 & 19087 & 0.14 & 0.12 & 0.19 & 0.79 & 0.80 \\
\hline WP-3D, May 16 & 21321 & 0.09 & 0.16 & 0.22 & 0.75 & 0.53 \\
\hline WP-3D, May 19 & 24221 & 0.15 & 0.06 & 0.15 & 0.80 & 0.87 \\
\hline WP-3D, May 21 & 10357 & 0.05 & 0.02 & 0.05 & 0.88 & 0.92 \\
\hline WP-3D, May 22 & 22536 & 0.03 & 0.05 & 0.08 & 0.55 & 0.60 \\
\hline WP-3D, May 23 & 20827 & 0.04 & 0.11 & 0.16 & 0.63 & 0.45 \\
\hline WP-3D, May 24 & 20661 & 0.07 & 0.14 & 0.22 & 0.44 & 0.46 \\
\hline WP-3D, May 25 & 20469 & 0.09 & 0.08 & 0.17 & 0.49 & 0.54 \\
\hline WP-3D, June 3 & 23570 & 0.16 & 0.07 & 0.15 & 0.66 & 0.77 \\
\hline WP-3D, June 14 & 26406 & 0.07 & 0.05 & 0.16 & 0.51 & 0.62 \\
\hline
\end{tabular}




\begin{tabular}{lcccccc} 
WP-3D, June 16 & 22634 & 0.07 & 0.04 & 0.08 & 0.75 & 0.78 \\
WP-3D, June 18 & 24707 & 0.05 & 0.04 & 0.07 & 0.77 & 0.83 \\
WP-3D, June 20 & 24681 & 0.07 & 0.14 & 0.23 & 0.67 & 0.46 \\
WP-3D, June 22 & 4907 & 0.07 & 0.08 & 0.15 & 0.67 & 0.72 \\
Twin Otter, May 6 & 179 & 0.16 & 0.34 & 0.37 & 0.51 & 0.27 \\
Twin Otter, May 7 & 194 & 0.22 & 0.36 & 0.51 & 0.61 & 0.30 \\
Twin Otter, May 10 & 186 & 0.07 & 0.19 & 0.21 & 0.51 & 0.22 \\
Twin Otter, May 12 & 195 & 0.14 & 0.24 & 0.32 & 0.63 & 0.37 \\
Twin Otter, May 13 & 178 & 0.12 & 0.31 & 0.48 & 0.26 & 0.19 \\
Twin Otter, May 14 & 189 & 0.15 & 0.44 & 0.48 & 0.42 & 0.23 \\
Twin Otter, May 15 & 185 & 0.16 & 0.34 & 0.39 & 0.35 & 0.22 \\
Twin Otter, May 18 & 183 & 0.03 & 0.07 & 0.09 & 0.62 & 0.41 \\
Twin Otter, May 19 & 116 & 0.18 & 0.39 & 0.43 & 0.25 & 0.25 \\
Twin Otter, May 20 & 192 & 0.06 & 0.08 & 0.12 & 0.92 & 0.69 \\
Twin Otter, May 21 & 192 & 0.15 & 0.20 & 0.25 & 0.33 & 0.33 \\
Twin Otter, May 22 & 195 & 0.03 & 0.04 & 0.07 & 0.76 & 0.47 \\
Twin Otter, May 24 & 173 & 0.07 & 0.08 & 0.09 & 0.89 & 0.68 \\
Twin Otter, May 25 & 191 & 0.10 & 0.12 & 0.14 & 0.80 & 0.53 \\
Twin Otter, May 27 & 176 & 0.11 & 0.20 & 0.23 & 0.08 & 0.21 \\
Twin Otter, May 28 & 192 & 0.16 & 0.20 & 0.37 & -0.12 & 0.13 \\
\hline
\end{tabular}


Table S38. Performance of simulated organic aerosol (OA) from the DEF_ANT simulation in terms of bias, RMSE, R, and IA for each for the G-1, WP-3D, and CIRPAS Twin Otter flights.

\begin{tabular}{|c|c|c|c|c|c|c|}
\hline Flight Date & $\begin{array}{c}\text { Number } \\
\text { of Data } \\
\text { Points }\end{array}$ & $\begin{array}{c}\text { Observed } \\
\text { Mean } \\
(\mu \mathrm{g} / \mathrm{m} 3) \\
\end{array}$ & $\begin{array}{c}\text { Bias } \\
(\mu \mathrm{g} / \mathrm{m} 3)\end{array}$ & RMSE & $\mathbf{R}$ & IA \\
\hline G-1, June $03 \mathrm{a}$ & 674 & 0.88 & -0.09 & 0.67 & 0.37 & 0.64 \\
\hline G-1. June 06a & 918 & 1.90 & -0.78 & 1.25 & 0.53 & 0.59 \\
\hline G-1, June $06 \mathrm{~b}$ & 510 & 2.05 & -0.94 & 1.66 & 0.13 & 0.48 \\
\hline G-1, June $08 \mathrm{a}$ & 946 & 4.02 & -1.67 & 2.30 & 0.76 & 0.65 \\
\hline G-1, June $08 \mathrm{~b}$ & 957 & 3.92 & -1.26 & 1.67 & 0.79 & 0.78 \\
\hline G-1, June 10a & 882 & 1.17 & -0.35 & 0.52 & 0.49 & 0.58 \\
\hline G-1, June $12 \mathrm{a}$ & 882 & 0.91 & -0.51 & 0.61 & 0.47 & 0.47 \\
\hline G-1, June $12 b$ & 793 & 2.17 & -1.54 & 1.66 & -0.33 & 0.29 \\
\hline G-1, June $14 \mathrm{a}$ & 928 & 4.08 & -2.32 & 2.65 & 0.52 & 0.47 \\
\hline G-1, June $15 a$ & 878 & 2.64 & -0.45 & 1.31 & 0.25 & 0.50 \\
\hline G-1, June $15 b$ & 882 & 4.93 & -0.97 & 1.99 & 0.46 & 0.60 \\
\hline G-1, June $18 \mathrm{a}$ & 922 & 2.14 & -0.36 & 2.13 & 0.12 & 0.35 \\
\hline G-1, June 19a & 887 & 2.16 & 0.30 & 1.00 & 0.45 & 0.66 \\
\hline G-1, June 21a & 898 & 3.05 & -1.55 & 2.48 & 0.45 & 0.49 \\
\hline G-1, June $21 b$ & 894 & 3.23 & -1.38 & 1.90 & 0.59 & 0.59 \\
\hline G-1, June $23 \mathrm{a}$ & 942 & 6.12 & -1.99 & 3.01 & 0.48 & 0.57 \\
\hline G-1, June $23 b$ & 874 & 6.25 & -0.81 & 2.17 & 0.53 & 0.64 \\
\hline G-1, June $24 \mathrm{a}$ & 934 & 2.07 & -0.22 & 1.84 & 0.13 & 0.45 \\
\hline G-1, June $24 b$ & 816 & 3.21 & -1.33 & 2.28 & 0.28 & 0.51 \\
\hline G-1, June $27 \mathrm{a}$ & 960 & 9.73 & -4.88 & 5.68 & 0.69 & 0.59 \\
\hline G-1, June $28 \mathrm{a}$ & 902 & 13.42 & -7.60 & 8.87 & 0.75 & 0.54 \\
\hline G-1, June $28 b$ & 668 & 10.83 & -7.02 & 8.45 & 0.60 & 0.47 \\
\hline WP-3D, May 4 & 1495 & 1.51 & 1.30 & 1.75 & 0.79 & 0.77 \\
\hline WP-3D, May 7 & 2383 & 2.02 & 0.36 & 1.03 & 0.84 & 0.90 \\
\hline WP-3D, May 8 & 2304 & 2.03 & 0.01 & 1.18 & 0.81 & 0.89 \\
\hline WP-3D, May 11 & 244 & 0.12 & 0.29 & 0.43 & 0.70 & 0.73 \\
\hline WP-3D, May 12 & - & - & - & - & - & - \\
\hline WP-3D, May 14 & 1765 & 1.92 & 1.18 & 1.91 & 0.70 & 0.76 \\
\hline WP-3D, May 16 & 2012 & 2.29 & 0.66 & 2.02 & 0.66 & 0.79 \\
\hline WP-3D, May 19 & 2102 & 1.85 & 0.20 & 1.21 & 0.87 & 0.91 \\
\hline WP-3D, May 21 & 434 & 1.14 & -0.37 & 1.23 & 0.79 & 0.78 \\
\hline WP-3D, May 22 & 1912 & 0.67 & 0.44 & 0.73 & 0.54 & 0.67 \\
\hline WP-3D, May 23 & 1906 & 1.04 & 0.30 & 0.71 & 0.66 & 0.75 \\
\hline WP-3D, May 24 & 1938 & 1.86 & 0.00 & 1.30 & 0.71 & 0.77 \\
\hline WP-3D, May 25 & 1879 & 1.52 & 0.39 & 0.97 & 0.48 & 0.67 \\
\hline WP-3D, June 3 & 2215 & 2.37 & 0.08 & 1.19 & 0.70 & 0.83 \\
\hline WP-3D, June 14 & 2438 & 1.88 & -0.22 & 1.62 & 0.74 & 0.83 \\
\hline
\end{tabular}




\begin{tabular}{lcccccc} 
WP-3D, June 16 & 2367 & 1.65 & 0.17 & 0.87 & 0.80 & 0.89 \\
WP-3D, June 18 & 2341 & 1.00 & 0.31 & 0.88 & 0.75 & 0.84 \\
WP-3D, June 20 & 2389 & 2.66 & -0.97 & 2.00 & 0.83 & 0.73 \\
WP-3D, June 22 & 481 & 1.01 & 0.94 & 1.51 & 0.29 & 0.51 \\
Twin Otter, May 18 & 183 & 1.80 & -0.58 & 1.31 & 0.69 & 0.74 \\
Twin Otter, May 19 & 146 & 3.51 & 0.77 & 1.16 & 0.51 & 0.62 \\
Twin Otter, May 20 & 192 & 1.30 & 0.40 & 0.71 & 0.92 & 0.94 \\
Twin Otter, May 21 & 192 & 2.27 & 0.55 & 0.88 & 0.68 & 0.74 \\
Twin Otter, May 22 & 195 & 0.93 & -0.19 & 0.44 & 0.86 & 0.90 \\
Twin Otter, May 24 & 173 & 1.50 & 0.17 & 0.67 & 0.64 & 0.78 \\
Twin Otter, May 25 & 191 & 1.10 & 1.32 & 1.54 & 0.45 & 0.43 \\
Twin Otter, May 27 & 176 & 2.00 & -0.39 & 0.76 & 0.15 & 0.47 \\
Twin Otter, May 28 & 192 & 2.27 & -0.09 & 0.96 & 0.05 & 0.43 \\
\hline
\end{tabular}


Table S39. Performance of simulated sulfate $\left(\mathrm{SO}_{4}{ }^{2-}\right)$ from the DEF_ANT simulation in terms of bias, RMSE, R, and IA for each for the G-1, WP-3D, and CIRPAS Twin Otter flights.

\begin{tabular}{|c|c|c|c|c|c|c|}
\hline Flight Date & $\begin{array}{c}\text { Number } \\
\text { of Data } \\
\text { Points }\end{array}$ & $\begin{array}{c}\text { Observed } \\
\text { Mean } \\
(\mu \mathrm{g} / \mathrm{m3})\end{array}$ & $\begin{array}{c}\text { Bias } \\
(\mu \mathrm{g} / \mathrm{m} 3)\end{array}$ & RMSE & $\mathbf{R}$ & IA \\
\hline G-1, June $03 \mathrm{a}$ & 674 & 0.51 & -0.04 & 0.24 & -0.16 & 0.28 \\
\hline G-1. June 06a & 918 & 0.32 & 0.06 & 0.23 & 0.16 & 0.35 \\
\hline G-1, June $06 \mathrm{~b}$ & 510 & 0.44 & -0.12 & 0.18 & -0.23 & 0.35 \\
\hline G-1, June $08 \mathrm{a}$ & 946 & 0.72 & -0.24 & 0.34 & 0.16 & 0.45 \\
\hline G-1, June $08 \mathrm{~b}$ & 957 & 0.88 & -0.20 & 0.37 & 0.59 & 0.70 \\
\hline G-1, June 10a & 882 & 0.25 & 0.06 & 0.13 & 0.30 & 0.52 \\
\hline G-1, June $12 \mathrm{a}$ & 882 & 0.13 & 0.17 & 0.18 & -0.11 & 0.30 \\
\hline G-1, June $12 b$ & 793 & 0.12 & 0.16 & 0.17 & -0.05 & 0.31 \\
\hline G-1, June $14 \mathrm{a}$ & 928 & 0.40 & 0.18 & 0.30 & 0.57 & 0.69 \\
\hline G-1, June $15 a$ & 878 & 0.54 & -0.08 & 0.17 & 0.56 & 0.60 \\
\hline G-1, June $15 b$ & 882 & 0.67 & -0.07 & 0.22 & 0.39 & 0.60 \\
\hline G-1, June $18 \mathrm{a}$ & 926 & 0.28 & 0.05 & 0.23 & 0.18 & 0.47 \\
\hline G-1, June 19a & 887 & 0.46 & 0.45 & 0.56 & 0.23 & 0.30 \\
\hline G-1, June 21a & 899 & 0.39 & 0.04 & 0.19 & -0.03 & 0.36 \\
\hline G-1, June $21 b$ & 894 & 0.39 & 0.04 & 0.19 & 0.39 & 0.60 \\
\hline G-1, June $23 \mathrm{a}$ & 944 & 0.58 & -0.13 & 0.29 & 0.19 & 0.47 \\
\hline G-1, June $23 b$ & 874 & 0.91 & -0.31 & 0.44 & 0.43 & 0.51 \\
\hline G-1, June $24 \mathrm{a}$ & 934 & 0.69 & -0.15 & 0.41 & 0.30 & 0.56 \\
\hline G-1, June $24 b$ & 816 & 0.65 & -0.06 & 0.39 & 0.30 & 0.59 \\
\hline G-1, June $27 \mathrm{a}$ & 960 & 0.75 & -0.04 & 0.32 & 0.63 & 0.71 \\
\hline G-1, June $28 \mathrm{a}$ & 902 & 0.72 & -0.13 & 0.39 & 0.17 & 0.42 \\
\hline G-1, June $28 b$ & 668 & 0.79 & -0.34 & 0.52 & 0.41 & 0.48 \\
\hline WP-3D, May 4 & 1495 & 0.42 & 0.17 & 0.29 & 0.70 & 0.76 \\
\hline WP-3D, May 7 & 2383 & 0.49 & -0.09 & 0.31 & 0.42 & 0.60 \\
\hline WP-3D, May 8 & 2304 & 0.78 & -0.18 & 0.76 & 0.18 & 0.38 \\
\hline WP-3D, May 11 & 244 & 0.32 & -0.03 & 0.22 & 0.36 & 0.61 \\
\hline WP-3D, May 12 & - & - & - & - & - & - \\
\hline WP-3D, May 14 & 1765 & 0.93 & -0.24 & 0.49 & 0.82 & 0.81 \\
\hline WP-3D, May 16 & 2012 & 1.19 & -0.20 & 0.53 & 0.73 & 0.82 \\
\hline WP-3D, May 19 & 2105 & 0.75 & -0.19 & 0.37 & 0.83 & 0.81 \\
\hline WP-3D, May 21 & 434 & 0.74 & -0.37 & 0.42 & 0.61 & 0.47 \\
\hline WP-3D, May 22 & 1912 & 0.51 & -0.24 & 0.32 & 0.20 & 0.44 \\
\hline WP-3D, May 23 & 1907 & 0.35 & 0.12 & 0.27 & 0.41 & 0.59 \\
\hline WP-3D, May 24 & 1938 & 0.59 & 0.05 & 0.28 & 0.83 & 0.90 \\
\hline WP-3D, May 25 & 1879 & 1.16 & -0.39 & 0.59 & 0.70 & 0.73 \\
\hline WP-3D, June 3 & 2215 & 1.26 & -0.44 & 0.72 & 0.51 & 0.63 \\
\hline WP-3D, June 14 & 2440 & 0.55 & -0.16 & 0.34 & 0.56 & 0.64 \\
\hline
\end{tabular}




\begin{tabular}{lcccccc} 
WP-3D, June 16 & 2368 & 0.78 & -0.41 & 0.51 & 0.67 & 0.59 \\
WP-3D, June 18 & 2341 & 0.48 & -0.14 & 0.28 & 0.64 & 0.69 \\
WP-3D, June 20 & 2389 & 0.99 & -0.41 & 0.56 & 0.62 & 0.66 \\
WP-3D, June 22 & 481 & 0.81 & -0.38 & 0.56 & 0.35 & 0.49 \\
Twin Otter, May 18 & 183 & 1.13 & -0.70 & 1.23 & -0.80 & 0.38 \\
Twin Otter, May 19 & 146 & 0.77 & 0.44 & 0.57 & 0.56 & 0.58 \\
Twin Otter, May 20 & 192 & 0.57 & -0.08 & 0.46 & -0.06 & 0.31 \\
Twin Otter, May 21 & 192 & 0.50 & 0.13 & 0.25 & 0.41 & 0.57 \\
Twin Otter, May 22 & 195 & 0.47 & -0.21 & 0.29 & 0.67 & 0.59 \\
Twin Otter, May 24 & 173 & 0.32 & -0.02 & 0.11 & 0.62 & 0.65 \\
Twin Otter, May 25 & 191 & 0.17 & 0.32 & 0.33 & 0.56 & 0.25 \\
Twin Otter, May 27 & 176 & 0.80 & -0.13 & 0.38 & 0.17 & 0.42 \\
Twin Otter, May 28 & 192 & 0.69 & -0.10 & 0.41 & 0.38 & 0.41 \\
\hline
\end{tabular}


Table S40. Performance of simulated nitrate $\left(\mathrm{NO}_{3}{ }^{-}\right)$from the DEF_ANT simulation in terms of bias, RMSE, R, and IA for each for the G-1, WP-3D, and CIRPAS Twin Otter flights.

\begin{tabular}{|c|c|c|c|c|c|c|}
\hline Flight Date & $\begin{array}{c}\text { Number } \\
\text { of Data } \\
\text { Points }\end{array}$ & $\begin{array}{c}\text { Observed } \\
\text { Mean } \\
(\mu \mathrm{g} / \mathrm{m} 3) \\
\end{array}$ & $\begin{array}{c}\text { Bias } \\
(\mu \mathrm{g} / \mathrm{m} 3)\end{array}$ & RMSE & $\mathbf{R}$ & IA \\
\hline G-1, June $03 \mathrm{a}$ & 674 & 0.12 & 0.00 & 0.11 & 0.07 & 0.37 \\
\hline G-1. June 06a & 918 & 0.09 & 0.01 & 0.06 & 0.33 & 0.56 \\
\hline G-1, June $06 \mathrm{~b}$ & 510 & 0.10 & -0.01 & 0.06 & 0.01 & 0.37 \\
\hline G-1, June $08 \mathrm{a}$ & 946 & 0.21 & -0.05 & 0.10 & -0.04 & 0.38 \\
\hline G-1, June $08 \mathrm{~b}$ & 957 & 0.21 & 0.02 & 0.10 & 0.59 & 0.73 \\
\hline G-1, June 10a & 882 & 0.07 & 0.02 & 0.05 & 0.07 & 0.39 \\
\hline G-1, June $12 \mathrm{a}$ & 882 & 0.05 & 0.06 & 0.07 & -0.08 & 0.40 \\
\hline G-1, June $12 b$ & 793 & 0.06 & 0.04 & 0.06 & -0.05 & 0.43 \\
\hline G-1, June $14 \mathrm{a}$ & 928 & 0.14 & 0.03 & 0.08 & 0.48 & 0.66 \\
\hline G-1, June $15 a$ & 878 & 0.17 & -0.02 & 0.10 & 0.29 & 0.55 \\
\hline G-1, June $15 b$ & 882 & 0.20 & 0.07 & 0.17 & 0.14 & 0.30 \\
\hline G-1, June $18 \mathrm{a}$ & 926 & 0.18 & -0.04 & 0.19 & 0.37 & 0.60 \\
\hline G-1, June 19a & 887 & 0.17 & 0.14 & 0.19 & 0.41 & 0.43 \\
\hline G-1, June 21a & 899 & 0.14 & 0.01 & 0.08 & 0.12 & 0.41 \\
\hline G-1, June $21 b$ & 894 & 0.12 & 0.02 & 0.07 & 0.45 & 0.62 \\
\hline G-1, June $23 \mathrm{a}$ & 944 & 0.21 & -0.04 & 0.11 & 0.04 & 0.40 \\
\hline G-1, June $23 b$ & 874 & 0.25 & -0.01 & 0.13 & 0.10 & 0.39 \\
\hline G-1, June $24 \mathrm{a}$ & 934 & 0.15 & 0.02 & 0.12 & 0.16 & 0.48 \\
\hline G-1, June $24 b$ & 816 & 0.14 & 0.04 & 0.14 & 0.28 & 0.53 \\
\hline G-1, June $27 \mathrm{a}$ & 960 & 0.21 & 0.02 & 0.08 & 0.62 & 0.75 \\
\hline G-1, June $28 \mathrm{a}$ & 902 & 0.24 & -0.04 & 0.10 & 0.44 & 0.55 \\
\hline G-1, June $28 b$ & 668 & 0.19 & -0.03 & 0.10 & 0.50 & 0.52 \\
\hline WP-3D, May 4 & 1495 & 0.61 & 0.47 & 2.05 & 0.57 & 0.63 \\
\hline WP-3D, May 7 & 2383 & 0.89 & 0.41 & 1.52 & 0.63 & 0.72 \\
\hline WP-3D, May 8 & 2304 & 0.65 & 0.22 & 1.60 & 0.63 & 0.68 \\
\hline WP-3D, May 11 & 244 & 0.11 & 0.09 & 0.28 & 0.84 & 0.86 \\
\hline WP-3D, May 12 & - & - & - & - & - & - \\
\hline WP-3D, May 14 & 1765 & 0.82 & 0.75 & 2.23 & 0.34 & 0.50 \\
\hline WP-3D, May 16 & 2012 & 1.19 & 0.57 & 1.62 & 0.80 & 0.87 \\
\hline WP-3D, May 19 & 2105 & 2.95 & -1.73 & 3.74 & 0.78 & 0.72 \\
\hline WP-3D, May 21 & 434 & 0.72 & -0.26 & 0.83 & 0.89 & 0.93 \\
\hline WP-3D, May 22 & 1912 & 0.09 & 0.00 & 0.26 & 0.37 & 0.56 \\
\hline WP-3D, May 23 & 1907 & 0.07 & -0.04 & 0.23 & 0.23 & 0.38 \\
\hline WP-3D, May 24 & 1938 & 0.29 & 0.50 & 1.79 & 0.64 & 0.49 \\
\hline WP-3D, May 25 & 1879 & 0.80 & -0.13 & 1.22 & 0.59 & 0.76 \\
\hline WP-3D, June 3 & 2215 & 1.26 & 0.14 & 2.33 & 0.56 & 0.72 \\
\hline WP-3D, June 14 & 2440 & 0.13 & -0.13 & 0.42 & 0.43 & 0.35 \\
\hline
\end{tabular}




\begin{tabular}{lcccccc} 
WP-3D, June 16 & 2368 & 0.36 & -0.06 & 0.64 & 0.52 & 0.69 \\
WP-3D, June 18 & 2341 & 0.30 & -0.16 & 0.50 & 0.65 & 0.79 \\
WP-3D, June 20 & 2389 & 0.45 & -0.02 & 1.09 & 0.58 & 0.66 \\
WP-3D, June 22 & 481 & 0.32 & -0.11 & 0.94 & 0.95 & 0.85 \\
Twin Otter, May 18 & 183 & 1.05 & -0.53 & 1.39 & 0.65 & 0.73 \\
Twin Otter, May 19 & 146 & 5.43 & -2.42 & 3.44 & 0.59 & 0.62 \\
Twin Otter, May 20 & 192 & 1.51 & -0.20 & 1.99 & 0.49 & 0.67 \\
Twin Otter, May 21 & 192 & 2.32 & -1.17 & 2.33 & 0.31 & 0.56 \\
Twin Otter, May 22 & 195 & 0.65 & 0.41 & 1.01 & 0.64 & 0.65 \\
Twin Otter, May 24 & 173 & 0.94 & -0.35 & 0.99 & 0.54 & 0.70 \\
Twin Otter, May 25 & 191 & 0.81 & 0.55 & 1.68 & 0.57 & 0.61 \\
Twin Otter, May 27 & 176 & 2.48 & -2.22 & 2.89 & 0.32 & 0.47 \\
Twin Otter, May 28 & 192 & 1.59 & 0.28 & 1.53 & 0.45 & 0.67 \\
\hline
\end{tabular}


Table S41. Performance of simulated ammonium $\left(\mathrm{NH}_{4}{ }^{+}\right)$from the DEF_ANT simulation in terms of bias, RMSE, R, and IA for each for the G-1, WP-3D, and CIRPAS Twin Otter flights.

\begin{tabular}{|c|c|c|c|c|c|c|}
\hline Flight Date & $\begin{array}{c}\text { Number } \\
\text { of Data } \\
\text { Points }\end{array}$ & $\begin{array}{c}\text { Observed } \\
\text { Mean } \\
(\mu \mathrm{g} / \mathrm{m} 3) \\
\end{array}$ & $\begin{array}{c}\text { Bias } \\
(\mu \mathrm{g} / \mathrm{m} 3)\end{array}$ & RMSE & $\mathbf{R}$ & IA \\
\hline G-1, June $03 \mathrm{a}$ & 674 & 0.09 & 0.04 & 0.21 & 0.11 & 0.25 \\
\hline G-1. June 06a & 918 & 0.13 & -0.13 & 0.16 & -0.22 & 0.43 \\
\hline G-1, June $06 \mathrm{~b}$ & 510 & 0.11 & -0.11 & 0.14 & 0.01 & 0.42 \\
\hline G-1, June $08 \mathrm{a}$ & 946 & 0.42 & -0.35 & 0.41 & -0.04 & 0.35 \\
\hline G-1, June $08 \mathrm{~b}$ & 957 & 0.29 & -0.27 & 0.34 & -0.01 & 0.43 \\
\hline G-1, June 10a & 882 & 0.11 & -0.11 & 0.15 & 0.06 & 0.38 \\
\hline G-1, June $12 \mathrm{a}$ & 882 & 0.06 & -0.06 & 0.07 & -0.03 & 0.39 \\
\hline G-1, June $12 b$ & 793 & 0.10 & -0.10 & 0.10 & - & 0.30 \\
\hline G-1, June $14 \mathrm{a}$ & 928 & 0.34 & -0.34 & 0.40 & 0.06 & 0.43 \\
\hline G-1, June $15 a$ & 878 & 0.47 & -0.24 & 0.40 & 0.40 & 0.58 \\
\hline G-1, June $15 b$ & 882 & 0.53 & -0.24 & 0.57 & 0.09 & 0.38 \\
\hline G-1, June $18 \mathrm{a}$ & 926 & 0.60 & -0.41 & 1.00 & 0.38 & 0.48 \\
\hline G-1, June 19a & 887 & 0.25 & -0.24 & 0.29 & 0.05 & 0.40 \\
\hline G-1, June 21a & 899 & 0.31 & -0.31 & 0.50 & 0.01 & 0.32 \\
\hline G-1, June $21 b$ & 894 & 0.19 & -0.19 & 0.24 & -0.11 & 0.42 \\
\hline G-1, June $23 \mathrm{a}$ & 944 & 0.40 & -0.38 & 0.48 & -0.11 & 0.30 \\
\hline G-1, June $23 b$ & 874 & 0.36 & -0.25 & 0.46 & -0.02 & 0.30 \\
\hline G-1, June $24 \mathrm{a}$ & 934 & 0.23 & -0.20 & 0.31 & -0.15 & 0.39 \\
\hline G-1, June $24 b$ & 816 & 0.25 & -0.25 & 0.29 & 0.01 & 0.41 \\
\hline G-1, June $27 \mathrm{a}$ & 960 & 0.43 & -0.43 & 0.47 & 0.19 & 0.39 \\
\hline G-1, June $28 \mathrm{a}$ & 902 & 0.61 & -0.61 & 0.69 & -0.14 & 0.40 \\
\hline G-1, June $28 b$ & 668 & 0.40 & -0.40 & 0.45 & -0.13 & 0.39 \\
\hline WP-3D, May 4 & 1495 & 0.36 & 0.12 & 0.67 & 0.57 & 0.70 \\
\hline WP-3D, May 7 & 2383 & 0.48 & 0.03 & 0.48 & 0.64 & 0.79 \\
\hline WP-3D, May 8 & 2304 & 0.50 & -0.10 & 0.56 & 0.57 & 0.71 \\
\hline WP-3D, May 11 & 244 & 0.10 & 0.01 & 0.10 & 0.83 & 0.90 \\
\hline WP-3D, May 12 & - & - & - & - & - & - \\
\hline WP-3D, May 14 & 1765 & 0.61 & 0.06 & 0.66 & 0.54 & 0.72 \\
\hline WP-3D, May 16 & 2012 & 0.86 & -0.01 & 0.55 & 0.82 & 0.90 \\
\hline WP-3D, May 19 & 2105 & 1.15 & -0.62 & 1.25 & 0.80 & 0.73 \\
\hline WP-3D, May 21 & 434 & 0.50 & -0.27 & 0.44 & 0.88 & 0.88 \\
\hline WP-3D, May 22 & 1912 & 0.20 & -0.11 & 0.19 & 0.18 & 0.49 \\
\hline WP-3D, May 23 & 1907 & 0.13 & 0.02 & 0.20 & 0.10 & 0.29 \\
\hline WP-3D, May 24 & 1938 & 0.31 & 0.13 & 0.50 & 0.73 & 0.75 \\
\hline WP-3D, May 25 & 1879 & 0.64 & -0.17 & 0.49 & 0.67 & 0.79 \\
\hline WP-3D, June 3 & 2215 & 0.80 & -0.11 & 0.74 & 0.63 & 0.78 \\
\hline WP-3D, June 14 & 2440 & 0.18 & -0.08 & 0.23 & 0.58 & 0.57 \\
\hline
\end{tabular}




\begin{tabular}{lcccccc} 
WP-3D, June 16 & 2368 & 0.37 & -0.19 & 0.32 & 0.61 & 0.72 \\
WP-3D, June 18 & 2341 & 0.21 & -0.11 & 0.24 & 0.75 & 0.79 \\
WP-3D, June 20 & 2389 & 0.45 & -0.17 & 0.44 & 0.56 & 0.69 \\
WP-3D, June 22 & 481 & 0.34 & -0.14 & 0.42 & 0.90 & 0.77 \\
Twin Otter, May 18 & 183 & 0.73 & -0.46 & 0.75 & 0.44 & 0.55 \\
Twin Otter, May 19 & 146 & 2.31 & -1.01 & 1.33 & 0.47 & 0.53 \\
Twin Otter, May 20 & 192 & 1.05 & -0.54 & 0.79 & 0.65 & 0.66 \\
Twin Otter, May 21 & 192 & 1.49 & -1.03 & 1.28 & 0.30 & 0.49 \\
Twin Otter, May 22 & 195 & 0.43 & -0.07 & 0.33 & 0.58 & 0.74 \\
Twin Otter, May 24 & 173 & 0.40 & -0.16 & 0.39 & 0.49 & 0.65 \\
Twin Otter, May 25 & 191 & 0.43 & 0.11 & 0.50 & 0.55 & 0.67 \\
Twin Otter, May 27 & 176 & 1.23 & -0.93 & 1.16 & 0.14 & 0.45 \\
Twin Otter, May 28 & 192 & 0.85 & -0.11 & 0.57 & 0.22 & 0.55 \\
\hline
\end{tabular}




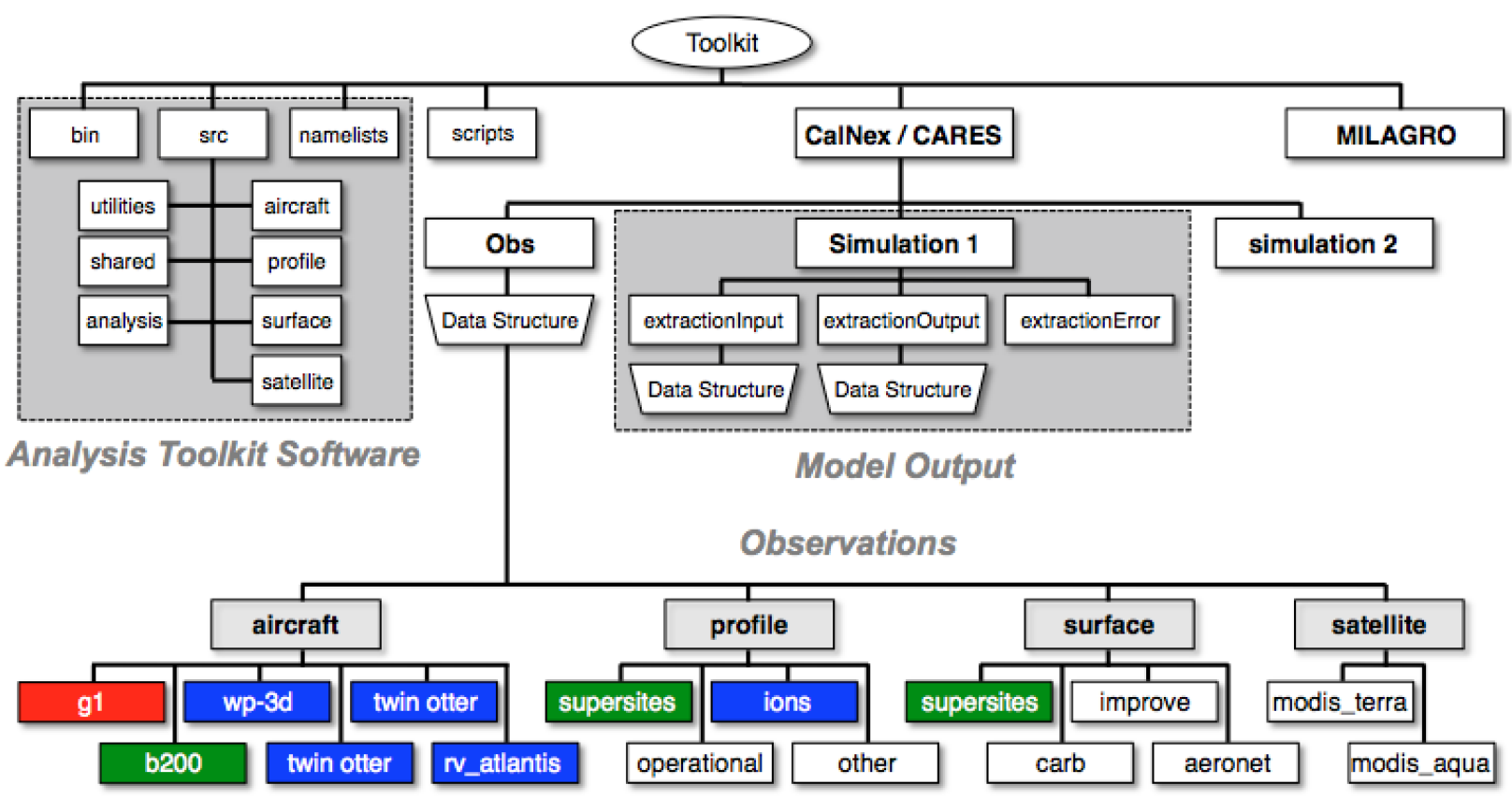

Figure S1. Directory structure of the Aerosol Modeling Testbed for the CalNex/CARES testbed case. Blue, red, and green denote data from CalNex, CARES, and both campaigns, respectively. 

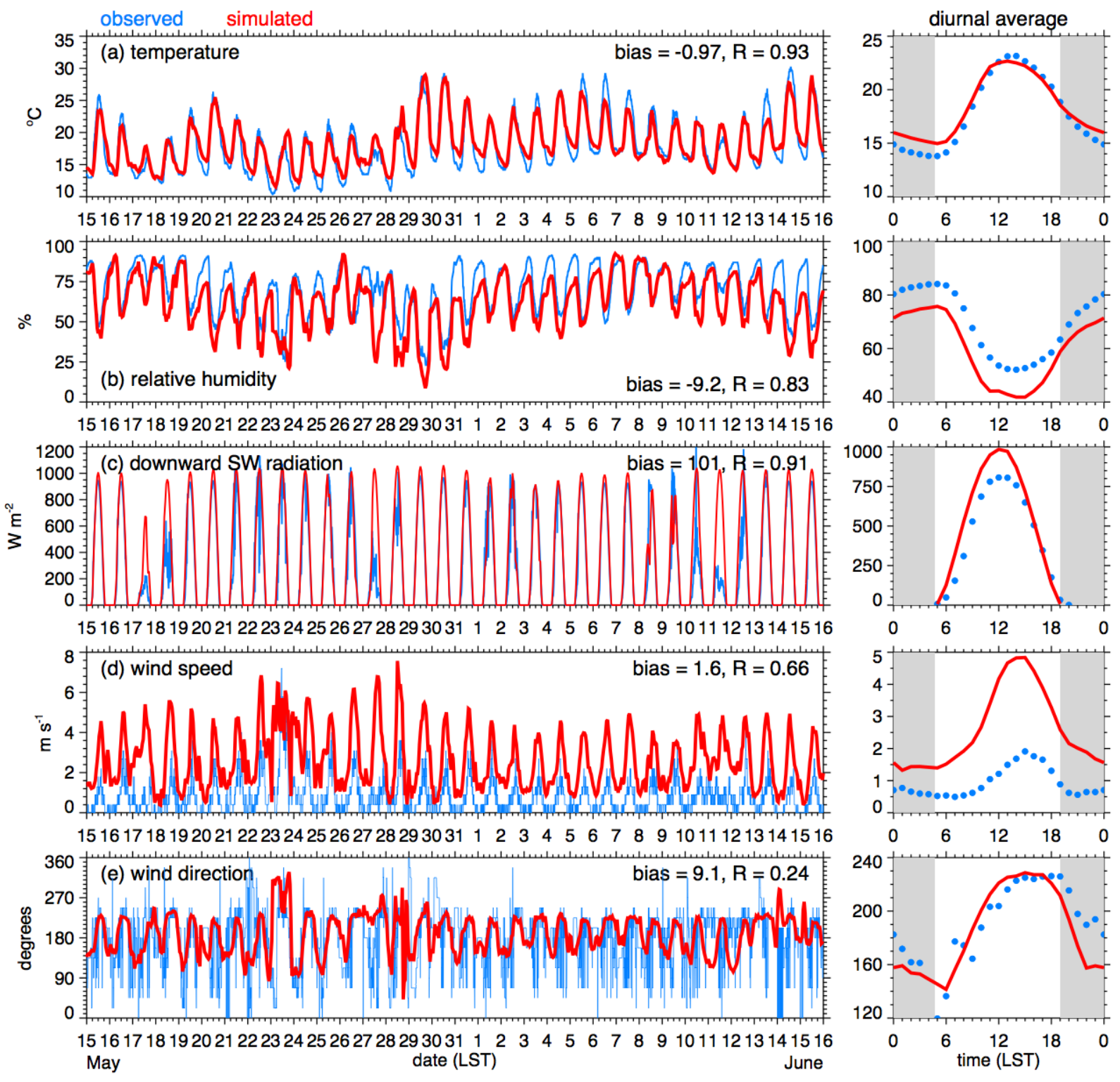

Figure S2. Time series (left panels) and diurnal average (right panels) of (a) temperature, (b) relative humidity, (c) downward shortwave radiation, (d) wind speed, and (e) wind direction at the Pasadena supersite. Observed values are hourly averages, while simulated values are instantaneous values at hourly intervals. Gray shading denotes night and $\mathrm{R}$ is the correlation coefficient. 
(a) SAC Wind Profiler, $970 \mathrm{~m}$ range gate

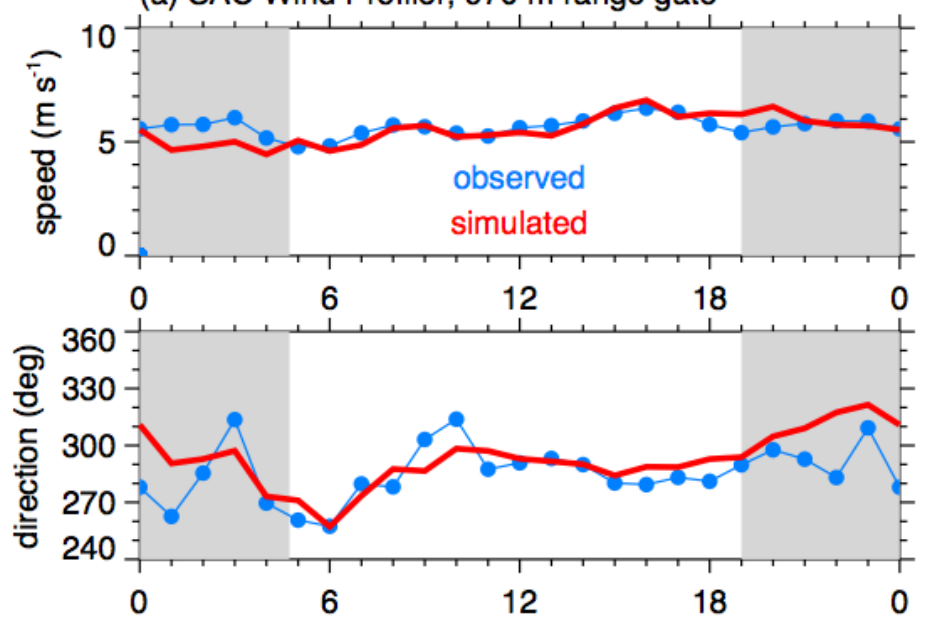

(b) BAK Radar Wind Profiler, $999 \mathrm{~m}$ range gate

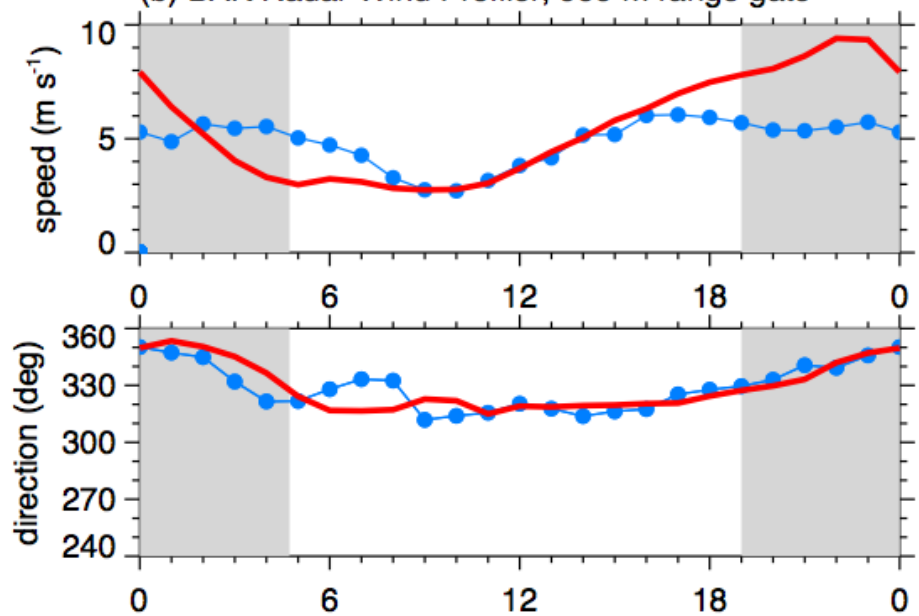

(c) USC Radar Wind Profiler, $1005 \mathrm{~m}$ range gate

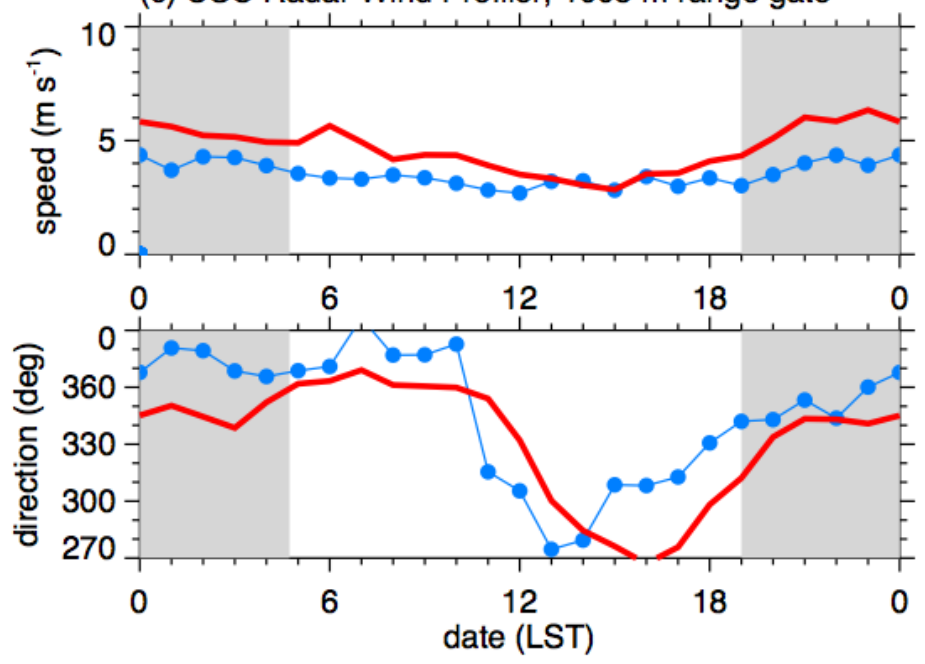

Figure S3. Observed and simulated diurnally-averaged wind speed and direction over the 2month period approximately $1 \mathrm{~km}$ AGL at the (a) SAC, (b) BAK, and (c) USC radar wind profiler sites. Gray shading denotes night 

(a) SAC Wind Profiler, $970 \mathrm{~m}$ range gate
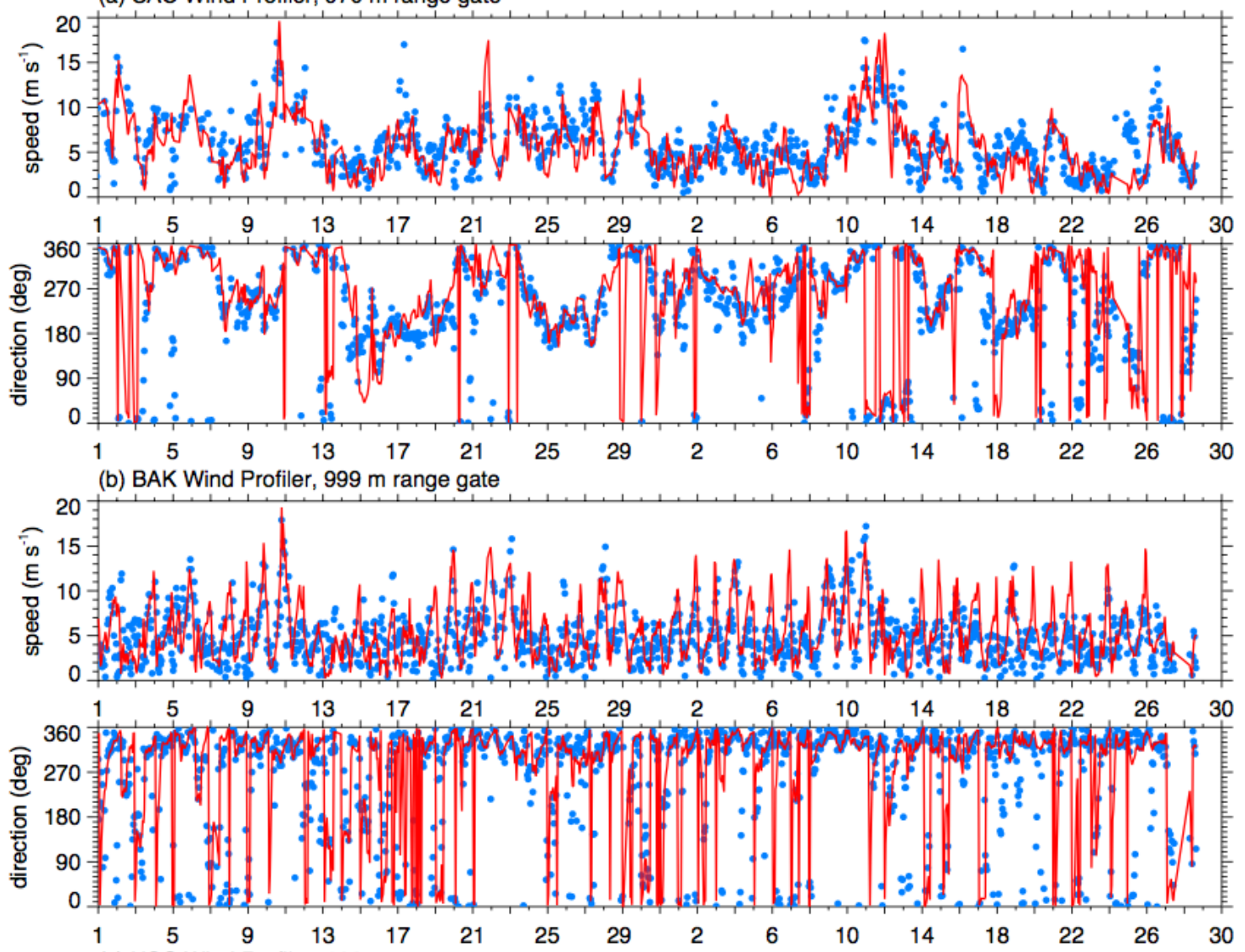

(c) USC Wind Profiler, $1005 \mathrm{~m}$ range gate
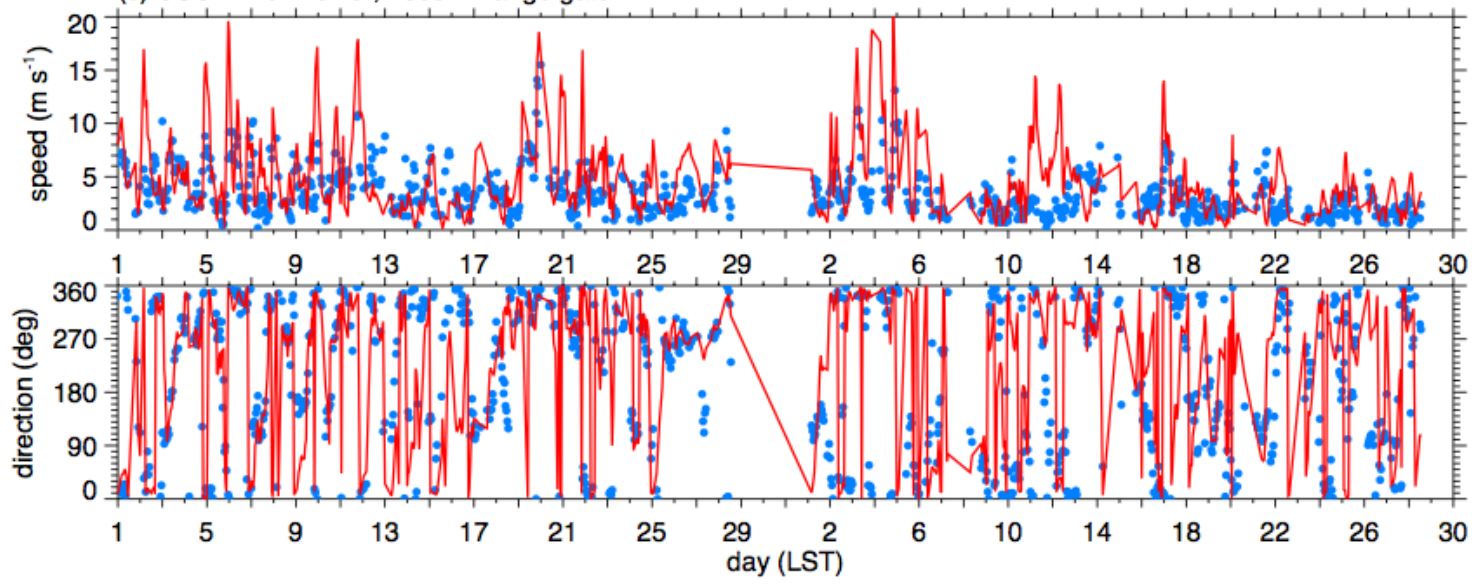

Figure S4. Comparison of observed and simulated wind speed and direction over May and June 2010 approximately $1 \mathrm{~km}$ AGL at the (a) SAC, (b), BAK, and (c) USC radar wind profiler sites. 


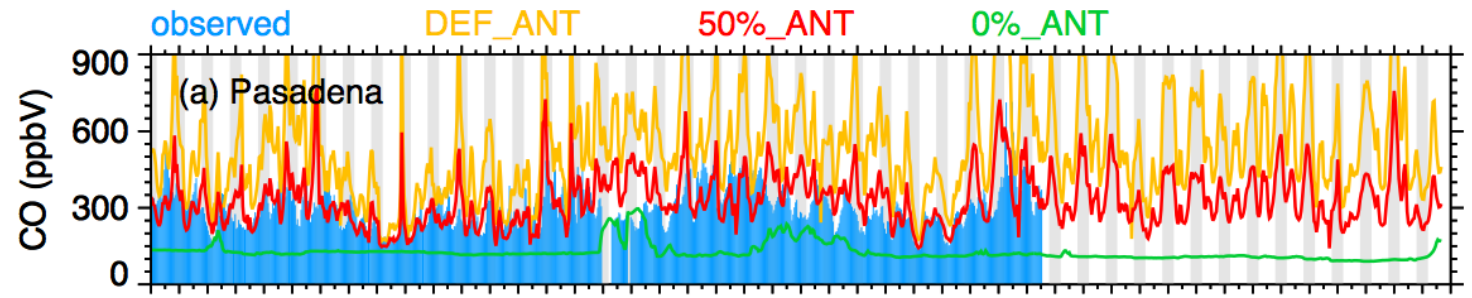

$\begin{array}{llllllllllllllllllllllll}15 & 17 & 19 & 21 & 23 & 25 & 27 & 29 & 31 & 2 & 4 & 6 & 8 & 10 & 12 & 14 & 16 & 18 & 20 & 22 & 24 & 26 & 28 & 30\end{array}$

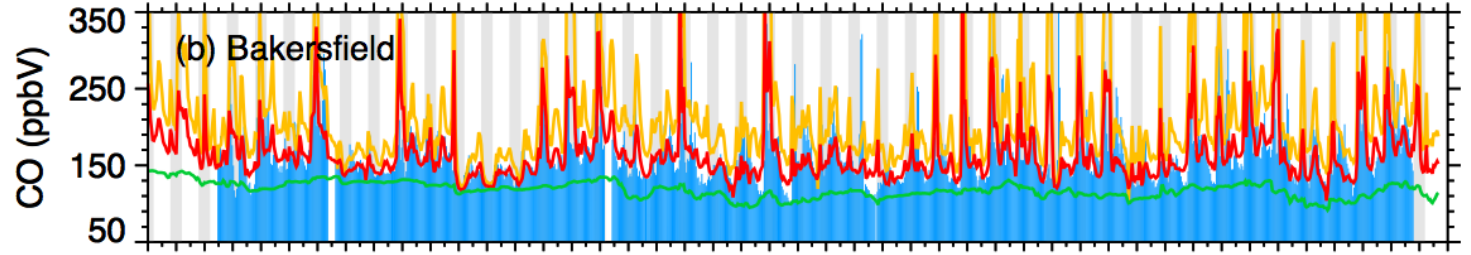

$\begin{array}{llllllllllllllllllllllll}15 & 17 & 19 & 21 & 23 & 25 & 27 & 29 & 31 & 2 & 4 & 6 & 8 & 10 & 12 & 14 & 16 & 18 & 20 & 22 & 24 & 26 & 28 & 30\end{array}$

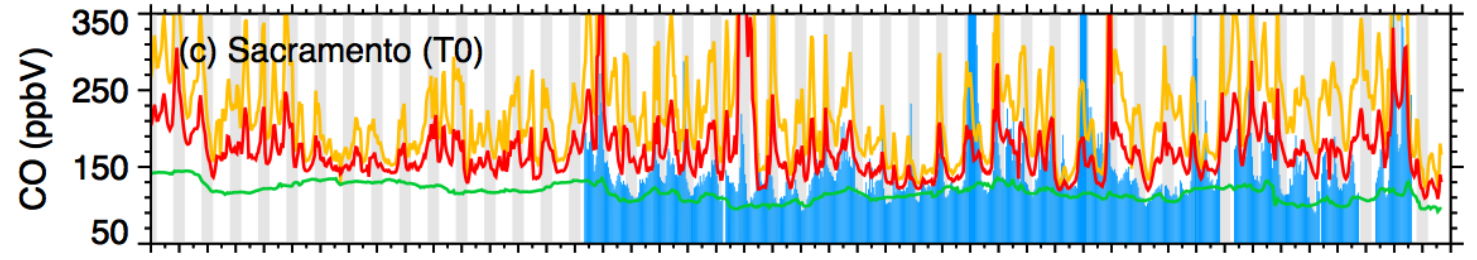

$\begin{array}{llllllllllllllllllllllll}15 & 17 & 19 & 21 & 23 & 25 & 27 & 29 & 31 & 2 & 4 & 6 & 8 & 10 & 12 & 14 & 16 & 18 & 20 & 22 & 24 & 26 & 28 & 30\end{array}$

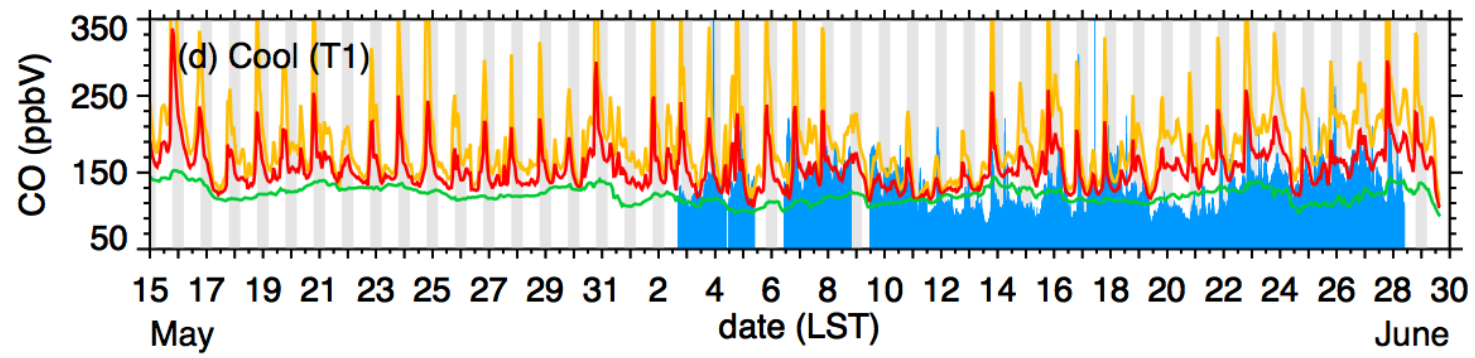

Figure S5. Observed and simulated carbon monoxide (CO) at the (a) Pasadena, (b) Bakersfield, (c) T0, and (d) T1 supersites. Gray shading denotes night. 


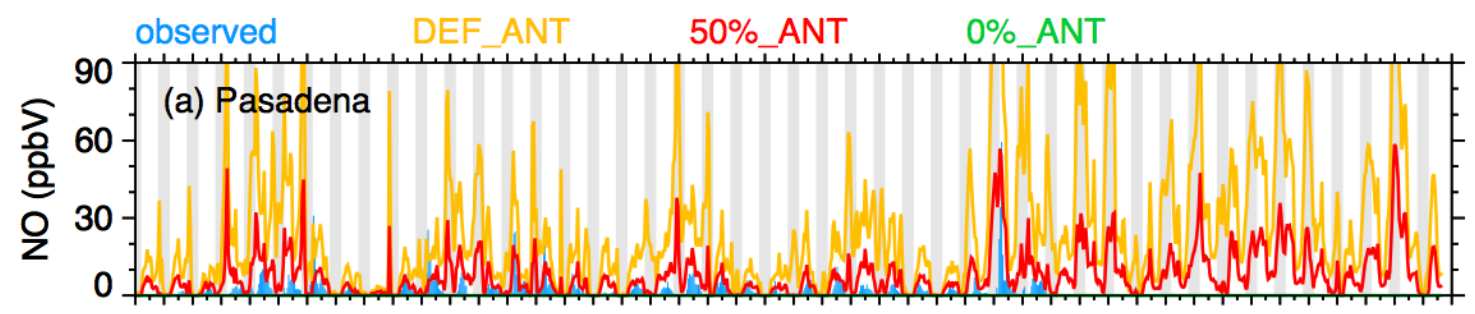

$\begin{array}{llllllllllllllllllllllll}15 & 17 & 19 & 21 & 23 & 25 & 27 & 29 & 31 & 2 & 4 & 6 & 8 & 10 & 12 & 14 & 16 & 18 & 20 & 22 & 24 & 26 & 28 & 30\end{array}$

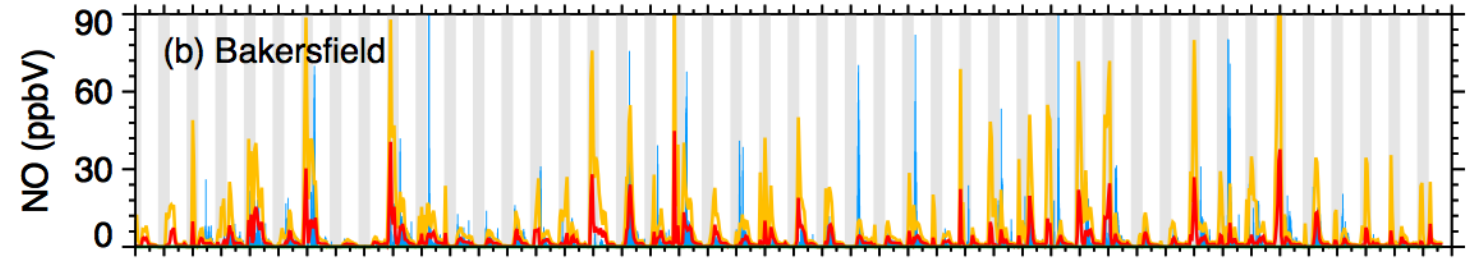

$\begin{array}{llllllllllllllllllllllll}15 & 17 & 19 & 21 & 23 & 25 & 27 & 29 & 31 & 2 & 4 & 6 & 8 & 10 & 12 & 14 & 16 & 18 & 20 & 22 & 24 & 26 & 28 & 30\end{array}$

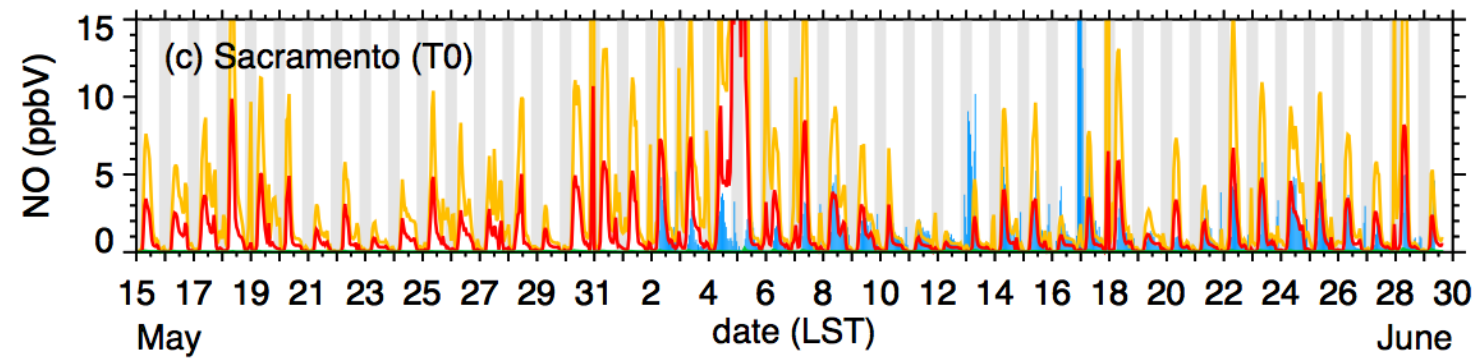

Figure S6. Observed and simulated nitrogen oxide (NO) at the (a) Pasadena, (b) Bakersfield, and (c) T0 supersites. Gray shading denotes night. 


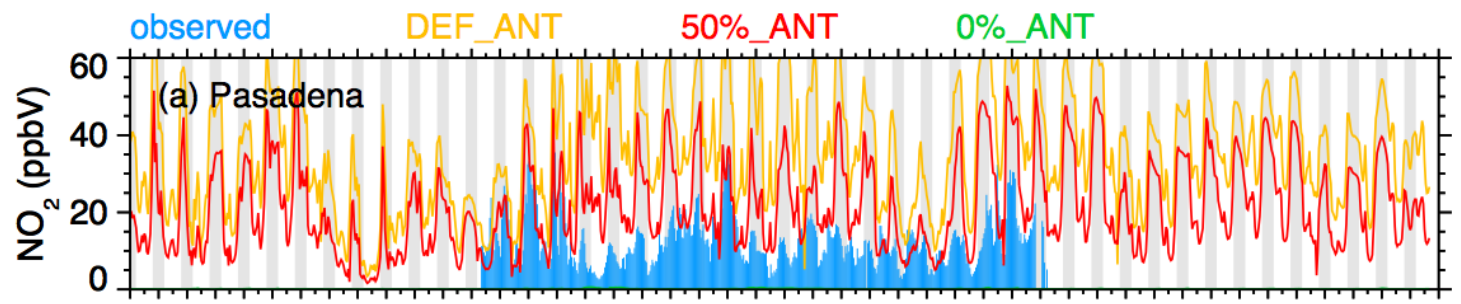

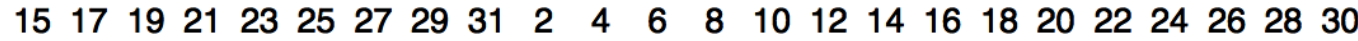

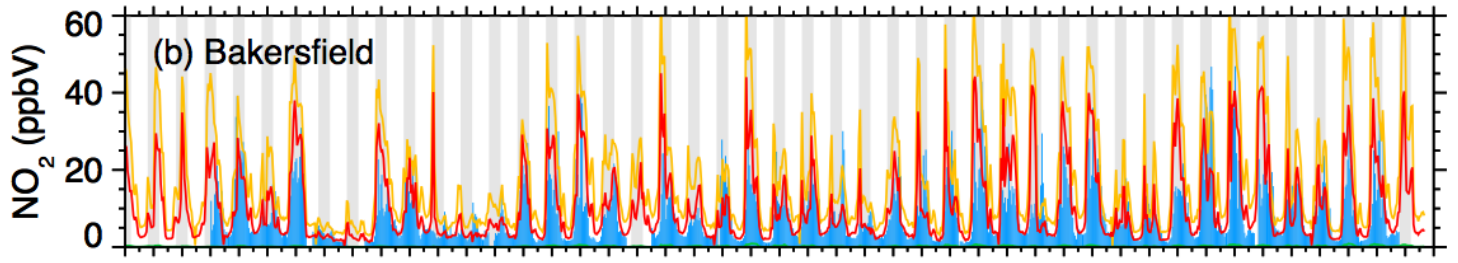

$\begin{array}{llllllllllllllllllllllll}15 & 17 & 19 & 21 & 23 & 25 & 27 & 29 & 31 & 2 & 4 & 6 & 8 & 10 & 12 & 14 & 16 & 18 & 20 & 22 & 24 & 26 & 28 & 30\end{array}$

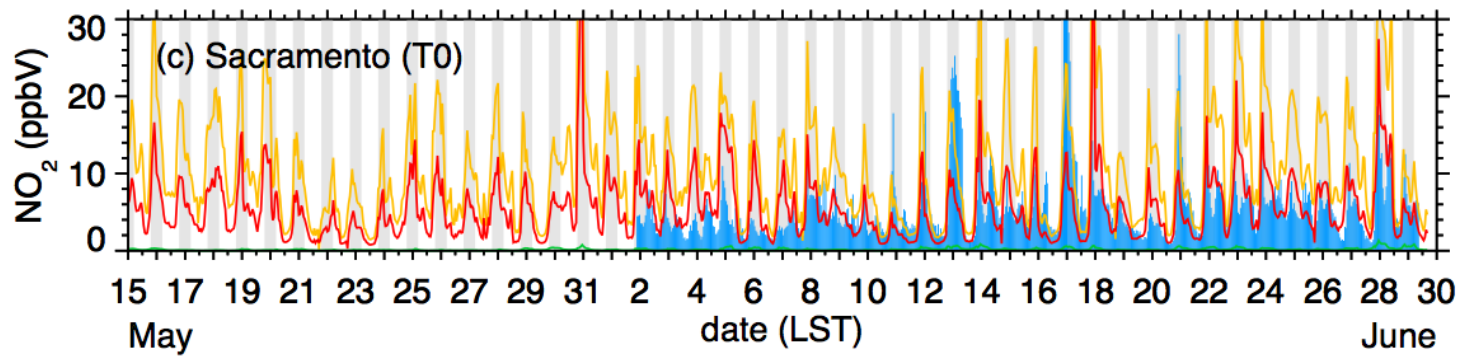

Figure S7. Observed and simulated nitrogen dioxide $\left(\mathrm{NO}_{2}\right)$ at the (a) Pasadena, (b) Bakersfield, and (c) T0 supersites. Gray shading denotes night. 

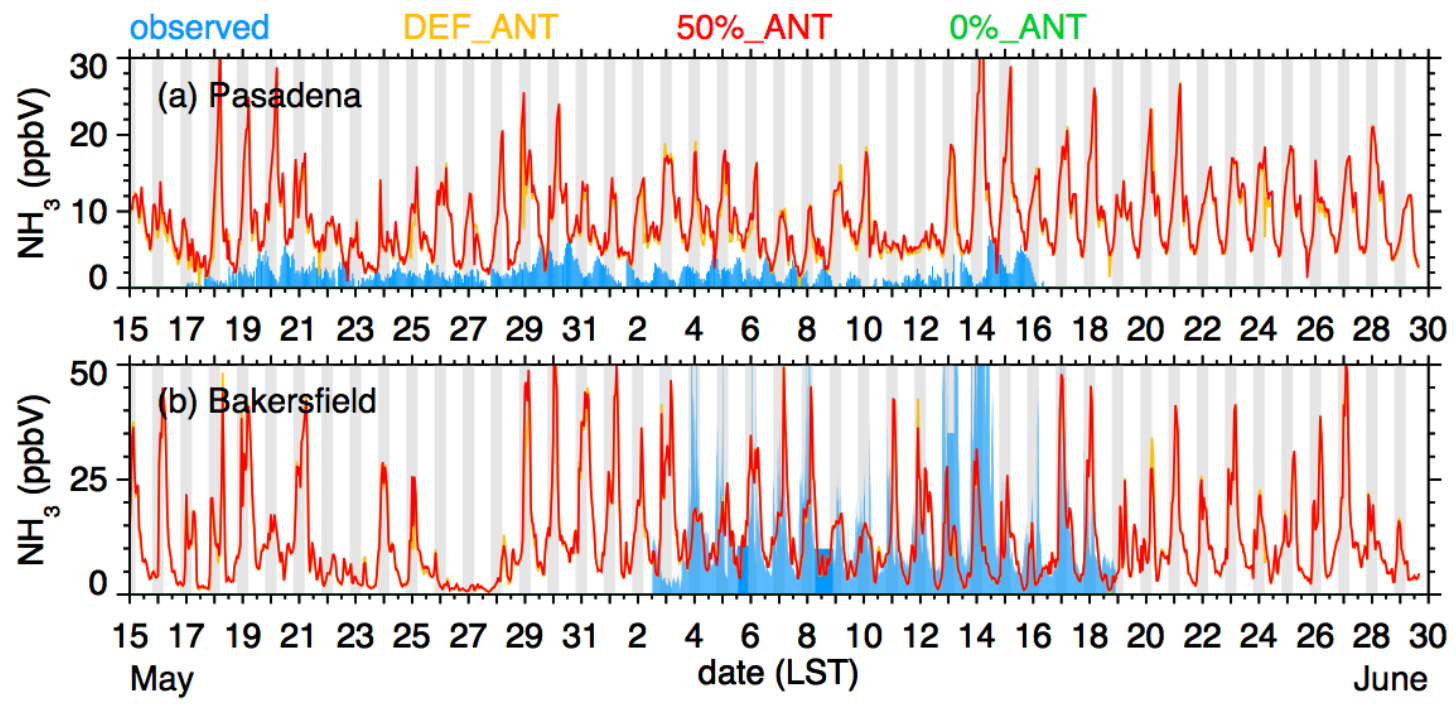

Figure S8. Observed and simulated ammonia $\left(\mathrm{NH}_{3}\right)$ at the (a) Pasadena and (b) Bakersfield supersites. Gray shading denotes night. 


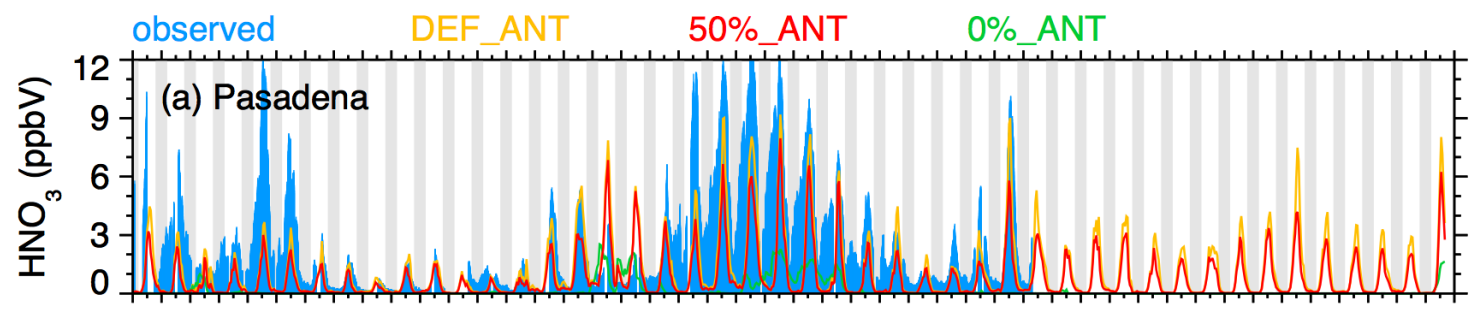

$\begin{array}{llllllllllllllllllllllll}15 & 17 & 19 & 21 & 23 & 25 & 27 & 29 & 31 & 2 & 4 & 6 & 8 & 10 & 12 & 14 & 16 & 18 & 20 & 22 & 24 & 26 & 28 & 30\end{array}$

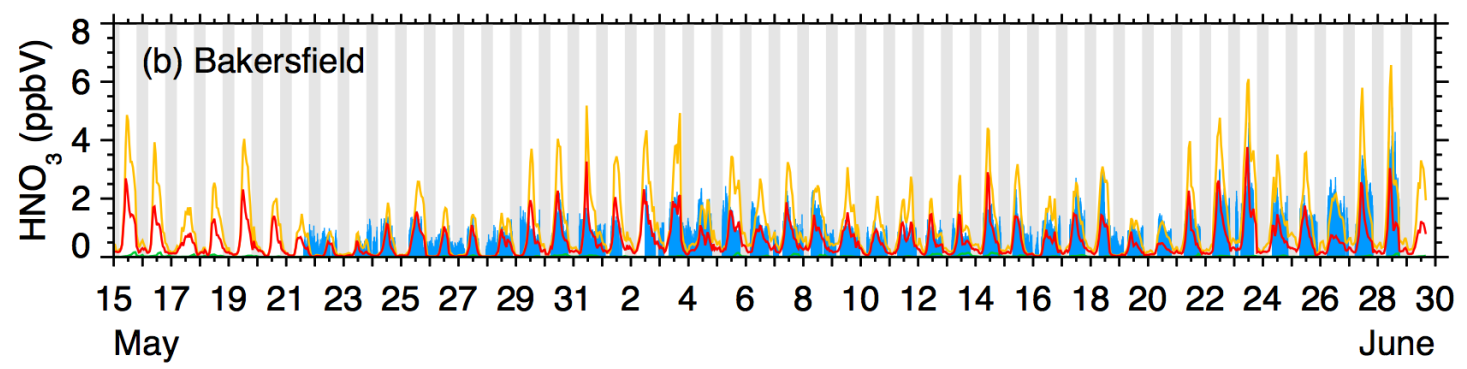

Figure S9. Observed and simulated nitric acid $\left(\mathrm{HNO}_{3}\right)$ at the (a) Pasadena and (b) Bakersfield supersites. Gray shading denotes night. 


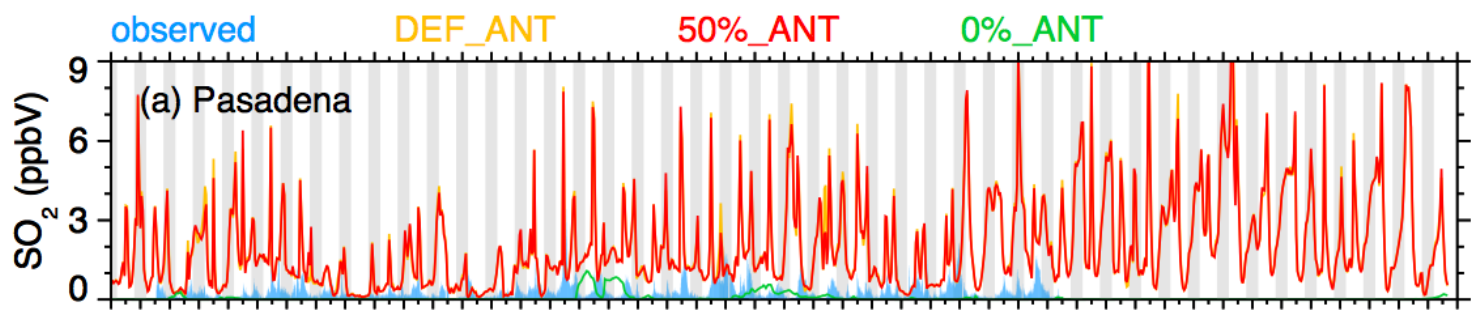

$\begin{array}{llllllllllllllllllllllll}15 & 17 & 19 & 21 & 23 & 25 & 27 & 29 & 31 & 2 & 4 & 6 & 8 & 10 & 12 & 14 & 16 & 18 & 20 & 22 & 24 & 26 & 28 & 30\end{array}$

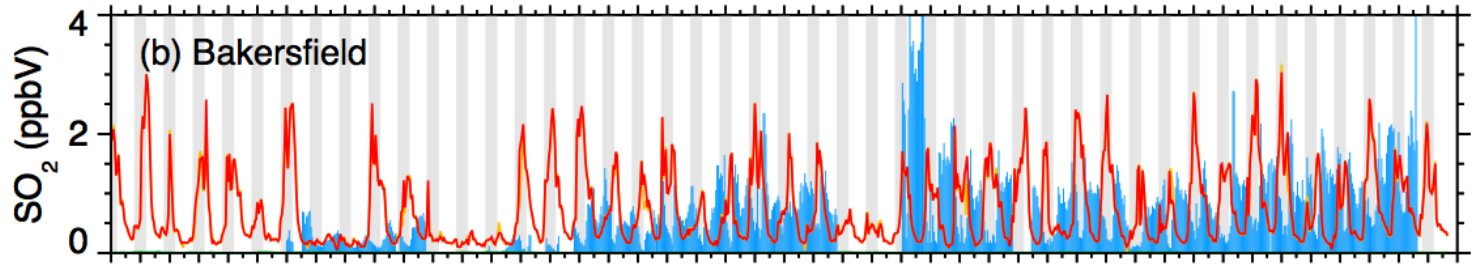

$\begin{array}{llllllllllllllllllllllll}15 & 17 & 19 & 21 & 23 & 25 & 27 & 29 & 31 & 2 & 4 & 6 & 8 & 10 & 12 & 14 & 16 & 18 & 20 & 22 & 24 & 26 & 28 & 30\end{array}$

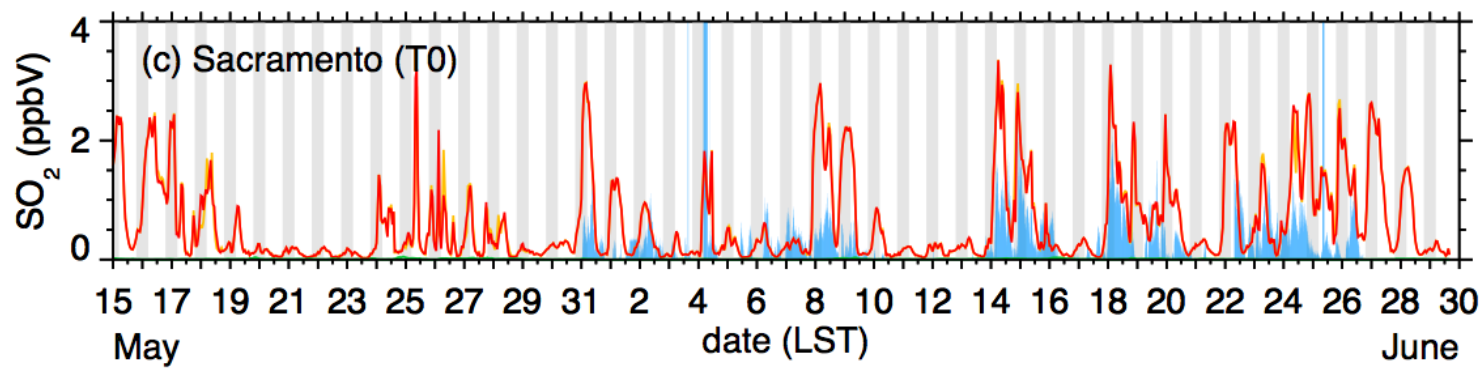

Figure S10. Observed and simulated sulfur dioxide $\left(\mathrm{SO}_{2}\right)$ at the (a) Pasadena, (b) Bakersfield, and (c) T0 supersites. Gray shading denotes night. 


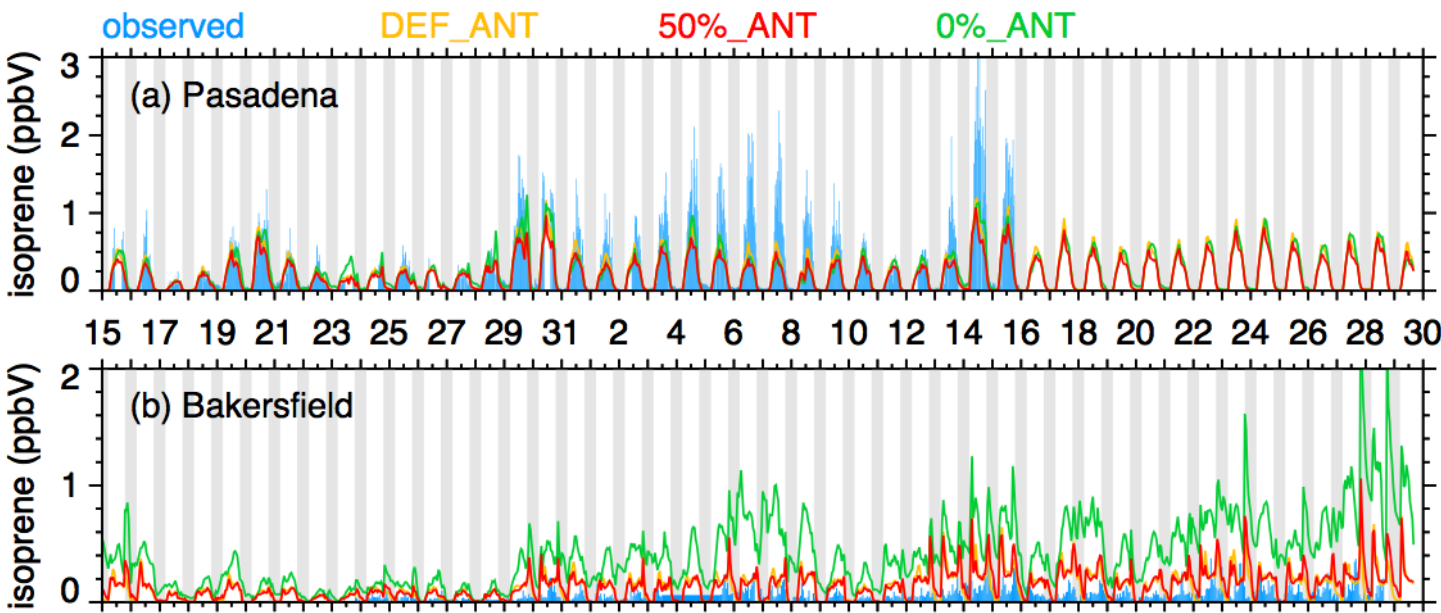

$\begin{array}{llllllllllllllllllllllll}15 & 17 & 19 & 21 & 23 & 25 & 27 & 29 & 31 & 2 & 4 & 6 & 8 & 10 & 12 & 14 & 16 & 18 & 20 & 22 & 24 & 26 & 28 & 30\end{array}$

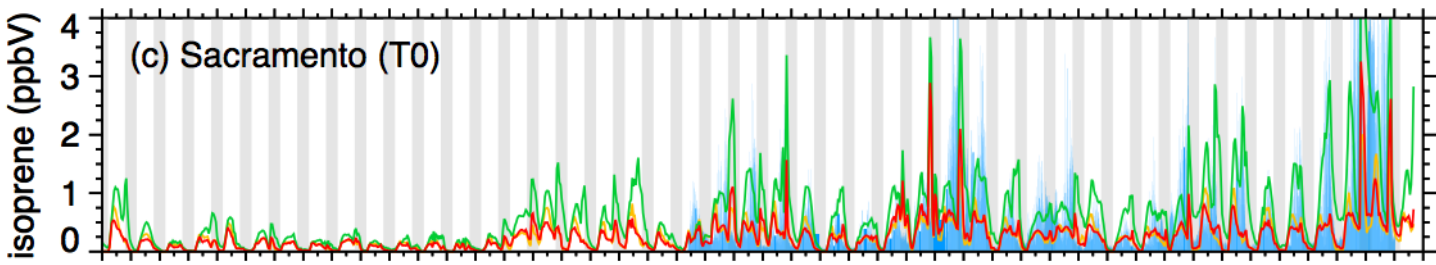

$\begin{array}{llllllllllllllllllllllll}15 & 17 & 19 & 21 & 23 & 25 & 27 & 29 & 31 & 2 & 4 & 6 & 8 & 10 & 12 & 14 & 16 & 18 & 20 & 22 & 24 & 26 & 28 & 30\end{array}$

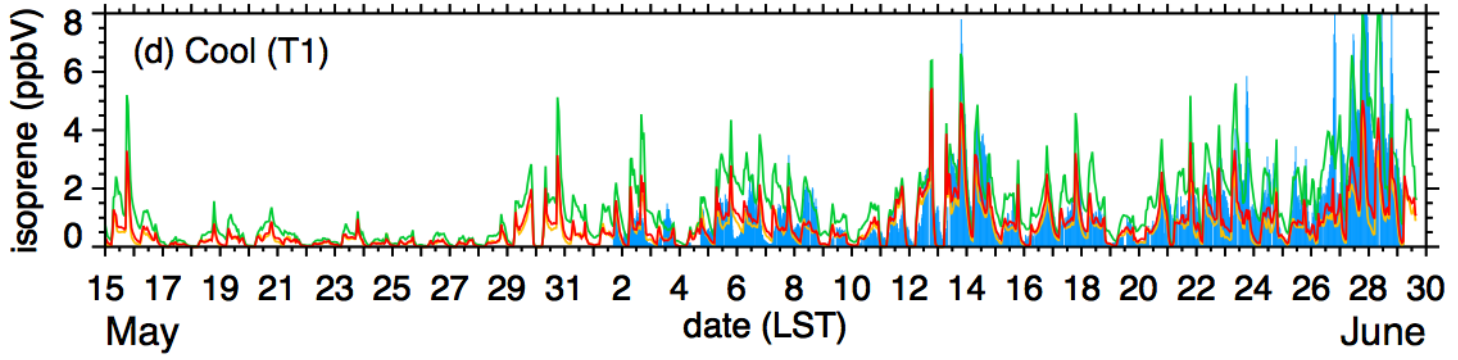

Figure S11. Observed and simulated isoprene at the (a) Pasadena, (b) Bakersfield, (c) T0, and (d) T1 supersites. Gray shading denotes night. 


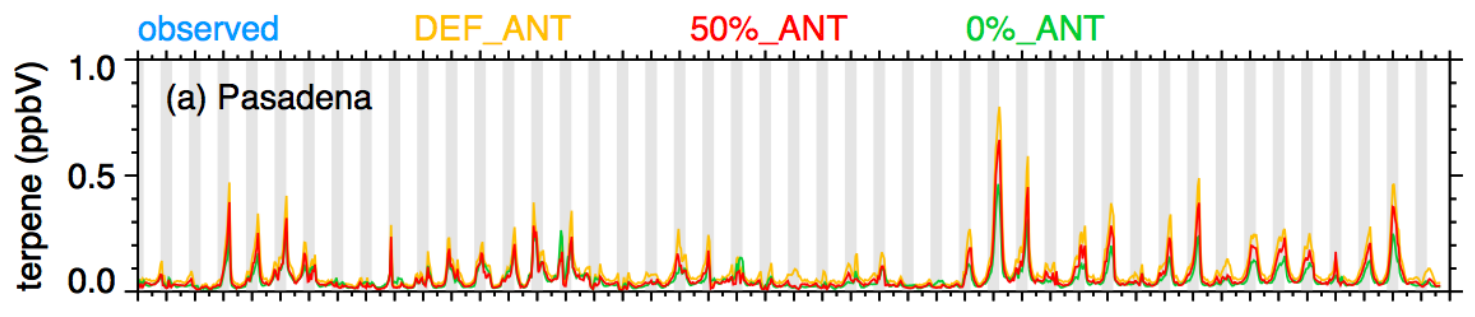

$\begin{array}{llllllllllllllllllllllll}15 & 17 & 19 & 21 & 23 & 25 & 27 & 29 & 31 & 2 & 4 & 6 & 8 & 10 & 12 & 14 & 16 & 18 & 20 & 22 & 24 & 26 & 28 & 30\end{array}$

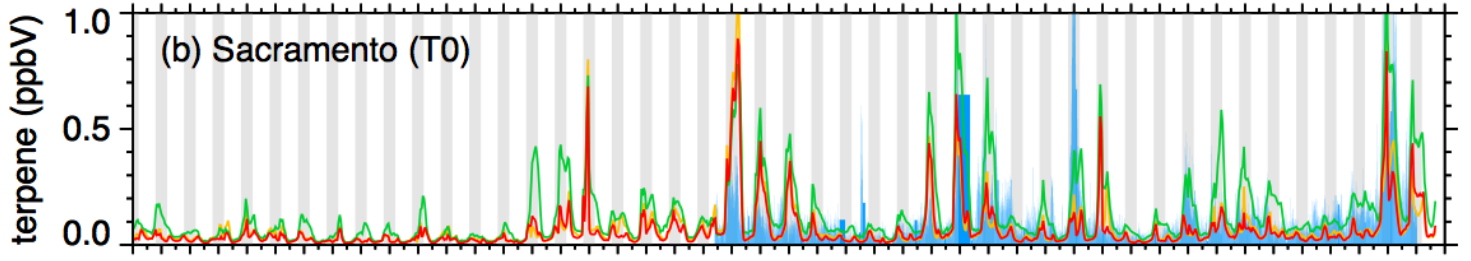

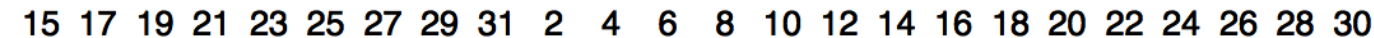

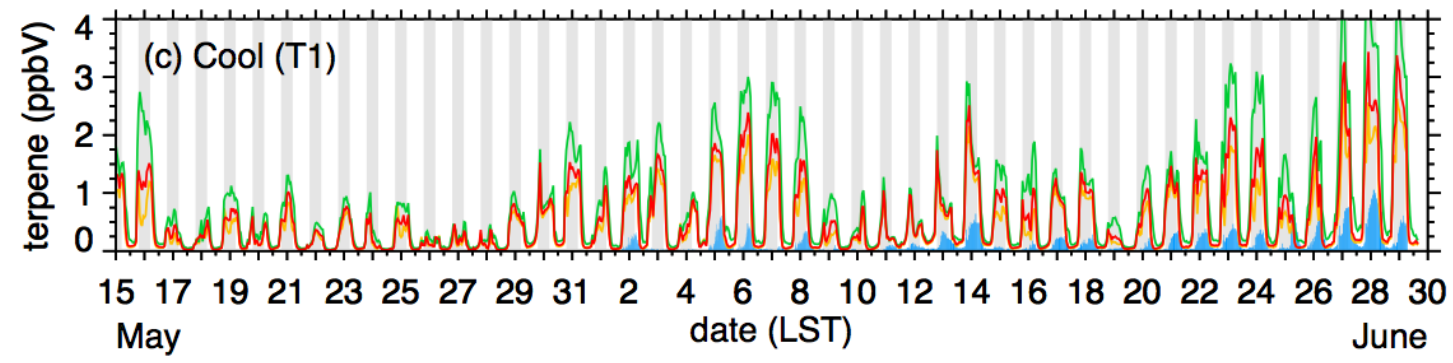

Figure S12. Observed and simulated terpene at the (a) Pasadena, (b) T0, and (c) T1 supersites. Gray shading denotes night. 

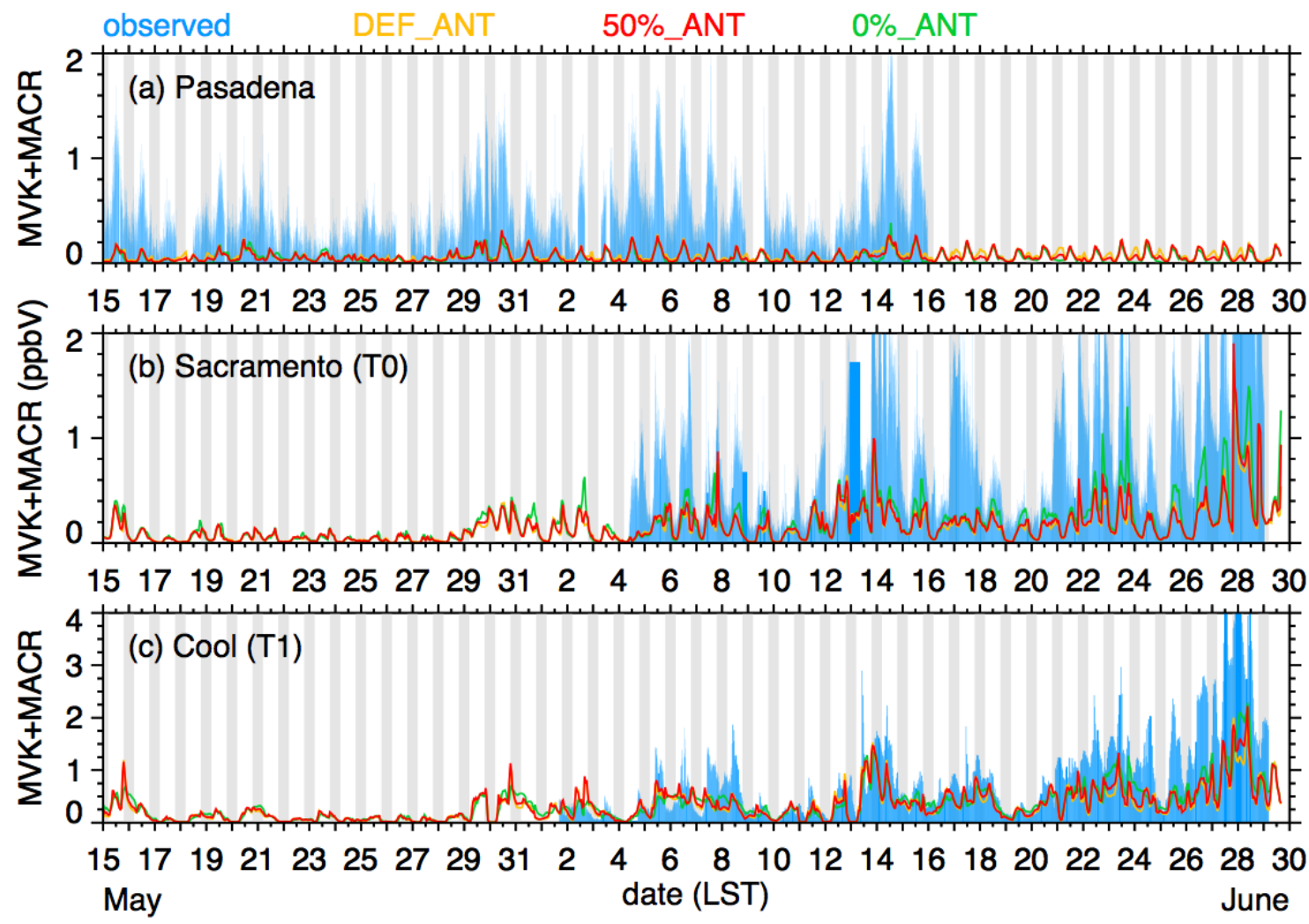

Figure S13. Observed and simulated methyl-vinyl-ketone + methacrolein (MVK+MACR) at the (a) Pasadena, (b) T0, and (c) T1 supersites. Gray shading denotes night. 

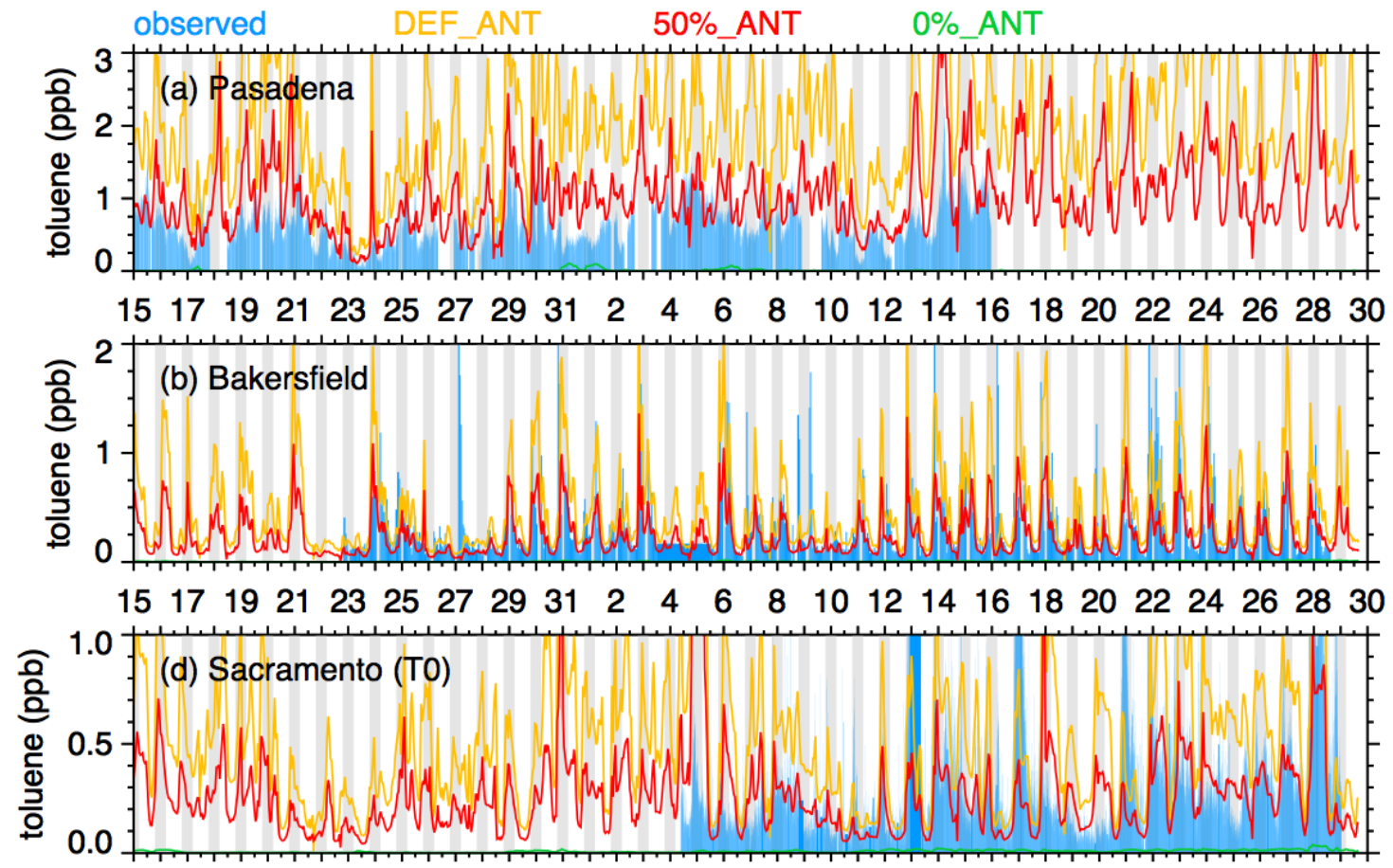

$\begin{array}{llllllllllllllllllllllll}15 & 17 & 19 & 21 & 23 & 25 & 27 & 29 & 31 & 2 & 4 & 6 & 8 & 10 & 12 & 14 & 16 & 18 & 20 & 22 & 24 & 26 & 28 & 30\end{array}$

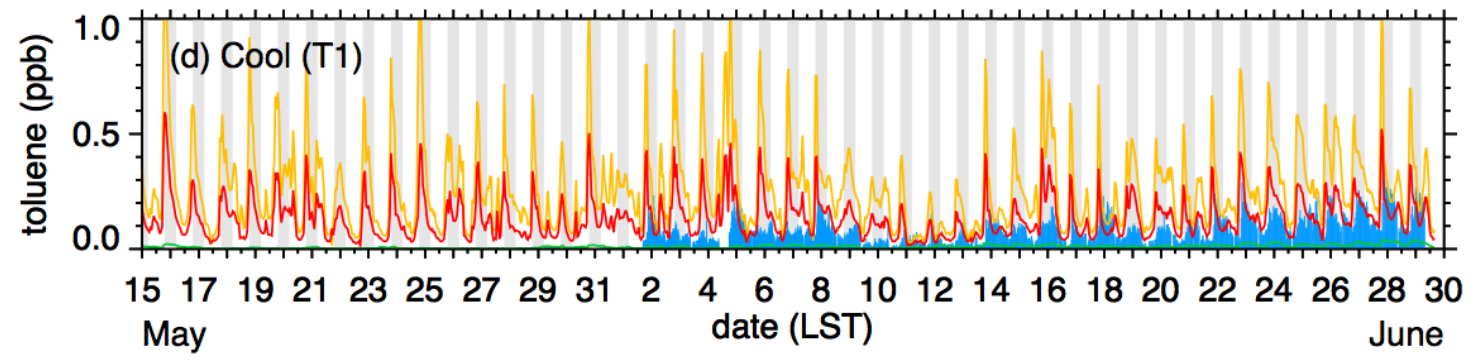

Figure S14. Observed and simulated toluene at the (a) Pasadena, (b) Bakersfield, (c) T0, and (d) T1 supersites. Gray shading denotes night. 


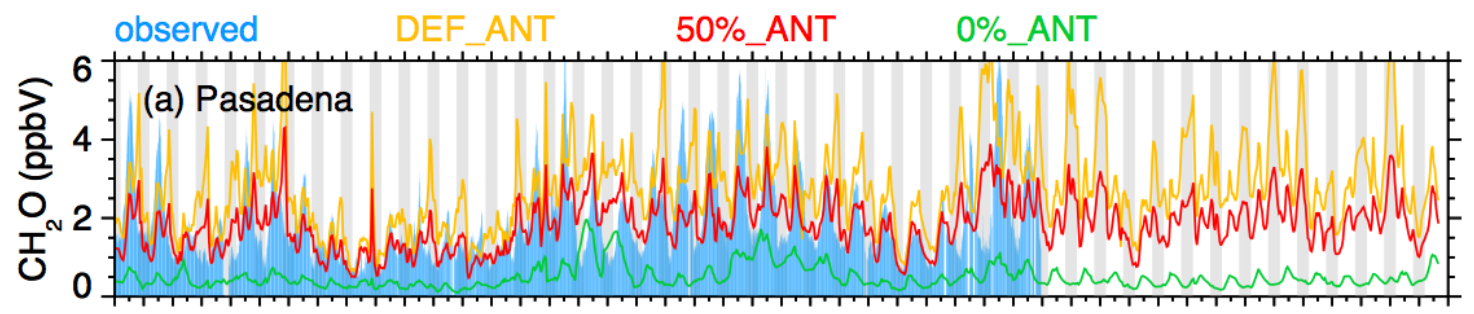

$\begin{array}{llllllllllllllllllllllll}15 & 17 & 19 & 21 & 23 & 25 & 27 & 29 & 31 & 2 & 4 & 6 & 8 & 10 & 12 & 14 & 16 & 18 & 20 & 22 & 24 & 26 & 28 & 30\end{array}$

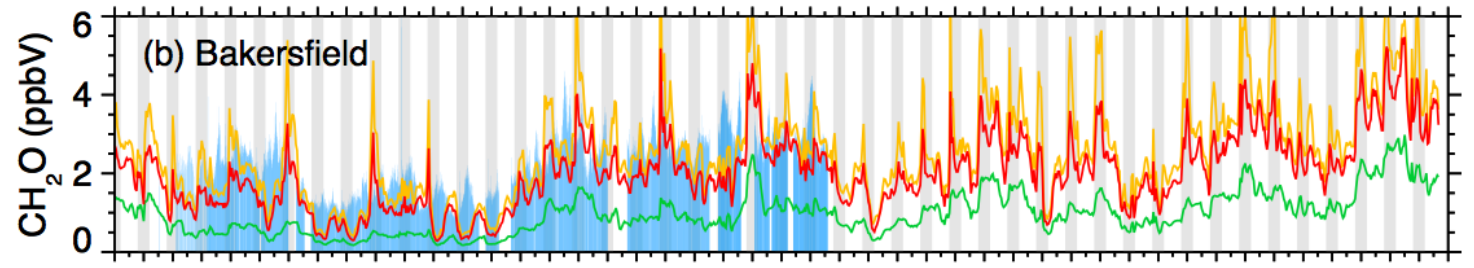

$\begin{array}{llllllllllllllllllllllll}15 & 17 & 19 & 21 & 23 & 25 & 27 & 29 & 31 & 2 & 4 & 6 & 8 & 10 & 12 & 14 & 16 & 18 & 20 & 22 & 24 & 26 & 28 & 30\end{array}$

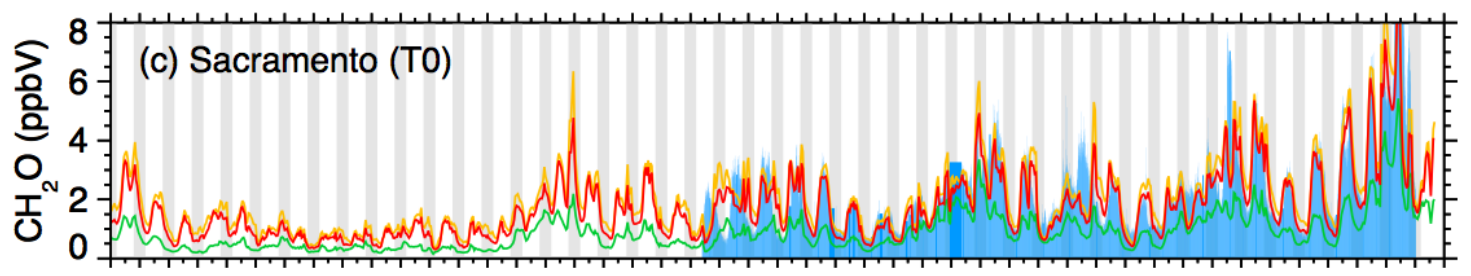

$\begin{array}{llllllllllllllllllllllll}15 & 17 & 19 & 21 & 23 & 25 & 27 & 29 & 31 & 2 & 4 & 6 & 8 & 10 & 12 & 14 & 16 & 18 & 20 & 22 & 24 & 26 & 28 & 30\end{array}$

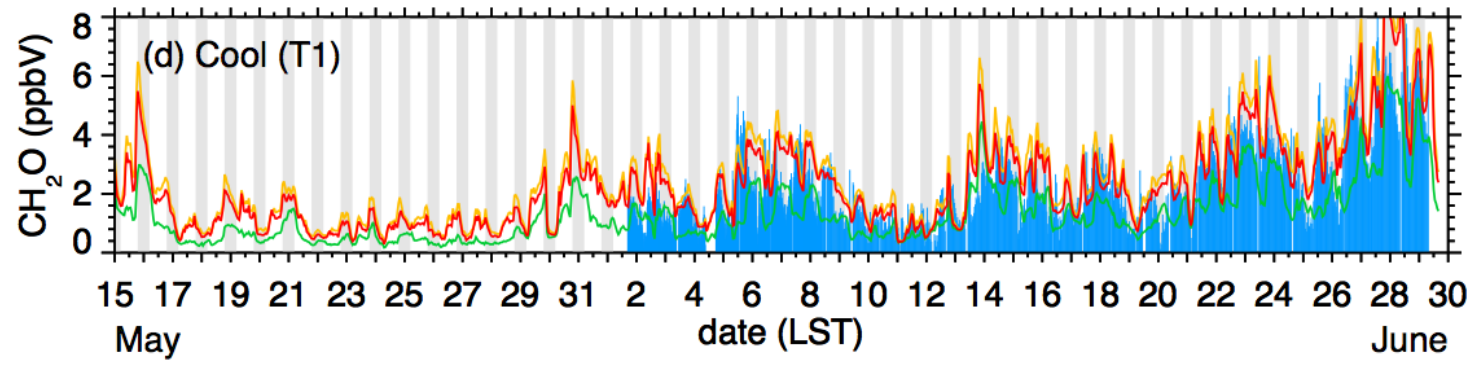

Figure S15. Observed and simulated formaldehyde $\left(\mathrm{CH}_{2} \mathrm{O}\right)$ at the (a) Pasadena, (b) Bakersfield, (c) T0, and (d) T1 supersites. Gray shading denotes night. 

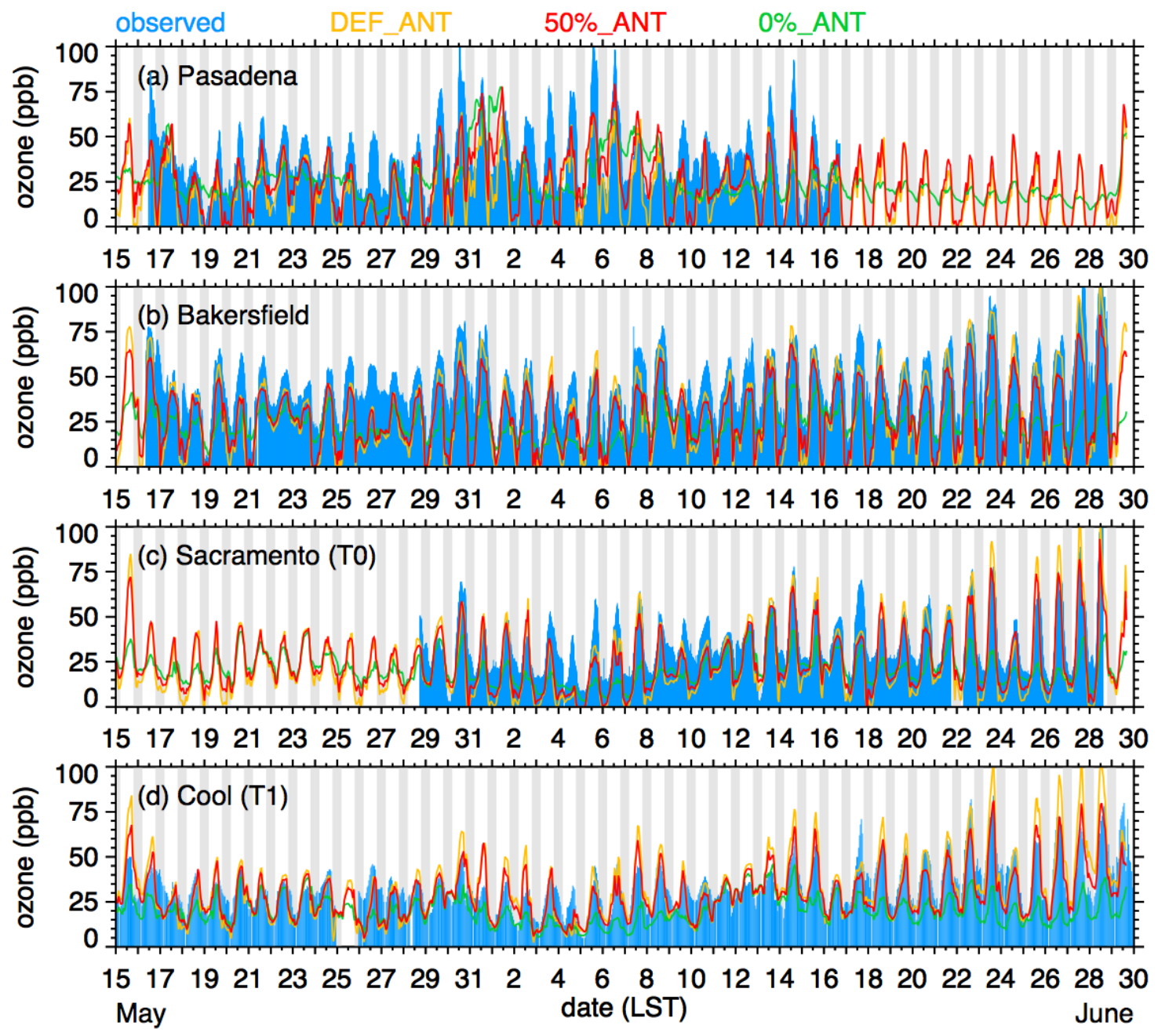

Figure S16. Observed and simulated ozone at the (a) Pasadena, (b) Bakersfield, (c) T0, and (d) T1 supersites. Gray shading denotes night. 